TRANSACTIONS OF THE

AMERICAN MATHEMATICAL SOCIETY

Volume 355, Number 12, Pages 5053-5089

S 0002-9947(03)03286-0

Article electronically published on July 24, 2003

\title{
THE FREE ENTROPY DIMENSION OF HYPERFINITE VON NEUMANN ALGEBRAS
}

\author{
KENLEY JUNG
}

For my parents

\begin{abstract}
Suppose $M$ is a hyperfinite von Neumann algebra with a normal, tracial state $\varphi$ and $\left\{a_{1}, \ldots, a_{n}\right\}$ is a set of selfadjoint generators for $M$. We calculate $\delta_{0}\left(a_{1}, \ldots, a_{n}\right)$, the modified free entropy dimension of $\left\{a_{1}, \ldots, a_{n}\right\}$. Moreover, we show that $\delta_{0}\left(a_{1}, \ldots, a_{n}\right)$ depends only on $M$ and $\varphi$. Consequently, $\delta_{0}\left(a_{1}, \ldots, a_{n}\right)$ is independent of the choice of generators for $M$. In the course of the argument we show that if $\left\{b_{1}, \ldots, b_{n}\right\}$ is a set of selfadjoint generators for a von Neumann algebra $\mathcal{R}$ with a normal, tracial state and $\left\{b_{1}, \ldots, b_{n}\right\}$ has finite-dimensional approximants, then $\delta_{0}(N) \leq \delta_{0}\left(b_{1}, \ldots, b_{n}\right)$ for any hyperfinite von Neumann subalgebra $N$ of $\mathcal{R}$. Combined with a result by Voiculescu, this implies that if $\mathcal{R}$ has a regular diffuse hyperfinite von Neumann subalgebra, then $\delta_{0}\left(b_{1}, \ldots, b_{n}\right)=1$.
\end{abstract}

\section{INTRODUCTION}

Suppose $G$ is a group. Consider the Hilbert space $L^{2}(G)$ where $G$ is endowed with counting measure and for each $g \in G$ write $u_{g}$ for the unitary operator on $L^{2}(G)$ defined by $\left(u_{g}(f)\right)(a)=f\left(g^{-1} a\right)$. Define the group von Neumann algebra $L(G)$ to be the von Neumann algebra generated by $\left\{u_{g}: g \in G\right\}$. It is not too hard to show that $L(G)$ is a factor (i.e, a von Neumann algebra such that if $x \in L(G)$ commutes with every other element in $L(G)$, then $x$ is a scalar multiple of the identity function) iff every nontrivial conjugacy class of $G$ is infinite. By definition the free group factor on $m$ generators is $L\left(F_{m}\right)$ where $F_{m}$ is the free group on $m$ generators.

Almost two decades ago Dan Voiculescu began to develop a noncommutative probability theory modeling the free group factors. The theory takes the notions of classical probability and transforms them into ones suited for noncommutative analysis. Random variables become elements in von Neumann algebras, expectations turn into normal, tracial states, and in this particular probability theory, independence always immediately follows the word "free". To clarify the last statement suppose $M$ is a von Neumann algebra with a normal, tracial state $\varphi$ and $\left\langle A_{j}\right\rangle_{j \in J}$ is a family of unital $*$-subalgebras of $M .\left\langle A_{j}\right\rangle_{j \in J}$ is a freely independent family provided that for any $j_{1}, \ldots, j_{p} \in J$ with $j_{1} \neq j_{2}, \ldots, j_{p-1} \neq j_{p}$, and $a_{i} \in A_{j_{i}}$,

$$
\varphi\left(a_{1}\right)=\cdots=\varphi\left(a_{p}\right)=0 \Rightarrow \varphi\left(a_{1} \cdots a_{p}\right)=0 .
$$

Received by the editors March 4, 2002 and, in revised form, January 9, 2003.

2000 Mathematics Subject Classification. Primary 46L54; Secondary 52C17, 53C30.

Research supported in part by the NSF. 
A family of subsets of $M$ is freely independent if the corresponding family of unital *-subalgebras that the subsets generate is freely independent. The definition generalizes the situation in $L\left(F_{m}\right) . L\left(F_{m}\right)$ has a unique normal, faithful, tracial state given by $\varphi(x)=\langle x \xi, \xi\rangle$ where $\xi$ is the characteristic function of the identity of $F_{m}$. If the generators for $F_{m}$ are $g_{1}, \ldots, g_{m}$, then one easily checks that $\left\{u_{g_{1}}\right\}^{\prime \prime}, \ldots,\left\{u_{g_{m}}\right\}^{\prime \prime}$ are freely independent. The parallels between classical and free probability go far and the interested reader can consult 8 for a general introduction.

On the operator algebra side, free probability has answered some open problems in operator algebras. Developing the ideas of free entropy and free entropy dimension, Voiculescu shows in [10] that the free group factors possess no Cartan subalgebras (the first known kind with separable predual). Ge shows in 3 . that the free group factors cannot be decomposed into a tensor product of two infinite-dimensional factors (again, the first known kind with separable predual) and similarly in [6] Stefan shows that the free group factors are not the 2-norm closure of the linear span of a product of abelian $*$-subalgebras.

In this paper we take a look at what free entropy dimension has in store for the most tractable kind of von Neumann algebras: those that are hyperfinite and have a tracial state. However, free entropy dimension being the nontrivial machine that it is, we review its definition and basic properties before stating our results.

1.1. Definitions and Properties. We recall the concepts of free entropy and modified free entropy dimension introduced in $\left[9\right.$. For $k, n \in \mathbb{N}$ write $M_{k}^{s a}(\mathbb{C})$ for the set of $k \times k$ selfadjoint matrices with complex entries and $\left(M_{k}^{s a}(\mathbb{C})\right)^{n}$ for the set of $n$-tuples of elements in $M_{k}^{s a}(\mathbb{C})$. Suppose $a_{1}, \ldots, a_{n} \in M$ are selfadjoint. Given $R>0, m, k \in \mathbb{N}$, and $\gamma>0$, define $\Gamma_{R}\left(a_{1}, \ldots, a_{n} ; m, k, \gamma\right)$ to be the set of $\left(x_{1}, \ldots, x_{n}\right) \in\left(M_{k}^{s a}(\mathbb{C})\right)^{n}$ such that for each $j,\left\|x_{j}\right\| \leq R$ and for any $1 \leq p \leq m$ and $1 \leq j_{1}, \ldots, j_{p} \leq n$,

$$
\left|\operatorname{tr}_{k}\left(x_{j_{1}} \cdots x_{j_{p}}\right)-\varphi\left(a_{j_{1}} \cdots a_{j_{p}}\right)\right|<\gamma
$$

Here $\operatorname{tr}_{k}$ denotes the tracial state on $M_{k}(\mathbb{C})$, the $k \times k$ matrices over $\mathbb{C}$. If $b_{1}, \ldots, b_{l} \in M$, then $\Gamma_{R}\left(a_{1}, \ldots, a_{n}: b_{1}, \ldots, b_{l} ; m, k, \gamma\right)$ denotes the set of all $\left(x_{1}, \ldots, x_{n}\right) \in\left(M_{k}^{s a}(\mathbb{C})\right)^{n}$ such that there exists a $\left(y_{1}, \ldots, y_{l}\right) \in\left(M_{k}^{s a}(\mathbb{C})\right)^{l}$ satisfying

$$
\left(x_{1}, \ldots, x_{n}, y_{1}, \ldots, y_{l}\right) \in \Gamma_{R}\left(a_{1}, \ldots, a_{n}, b_{1}, \ldots, b_{l} ; m, k, \gamma\right) .
$$

For any $d \in \mathbb{N}$ denote by vol the Lebesgue measure on $\left(M_{k}^{s a}(\mathbb{C})\right)^{d}$ (or a subspace thereof) with respect to the unnormalized Hilbert-Schmidt norm $\left\|\left(z_{1}, \ldots, z_{d}\right)\right\|_{2}=$ $\left(\sum_{j=1}^{d} \operatorname{Tr}\left(z_{j}^{2}\right)\right)^{\frac{1}{2}}$ where $\operatorname{Tr}$ is the unnormalized trace. One successively defines for any $R, \gamma>0$ and $m, k \in \mathbb{N}$,

$$
\begin{gathered}
\chi_{R}\left(a_{1}, \ldots, a_{n}: b_{1}, \ldots, b_{l} ; m, k, \gamma\right) \\
=k^{-2} \cdot \log \left(\operatorname{vol}\left(\Gamma_{R}\left(a_{1}, \ldots, a_{n}: b_{1}, \ldots, b_{l} ; m, k, \gamma\right)\right)\right)+\frac{n}{2} \log k, \\
\chi_{R}\left(a_{1}, \ldots, a_{n}: b_{1}, \ldots, b_{l} ; m, \gamma\right)=\limsup _{k \rightarrow \infty} \chi_{R}\left(a_{1}, \ldots, z_{n}: b_{1}, \ldots, b_{l} ; m, k, \gamma\right), \\
\chi_{R}\left(a_{1}, \ldots, a_{n}: b_{1}, \ldots, b_{l}\right)=\inf \left\{\chi_{R}\left(a_{1}, \ldots, a_{n}: b_{1}, \ldots, b_{l} ; m, \gamma\right): m \in \mathbb{N}, \gamma>0\right\}, \\
\chi\left(a_{1}, \ldots, a_{n}: b_{1}, \ldots, b_{l}\right)=\sup _{R>0} \chi_{R}\left(a_{1}, \ldots, a_{n}: b_{1}, \ldots, b_{l}\right) .
\end{gathered}
$$

$\chi\left(a_{1}, \ldots, a_{n}: b_{1}, \ldots, b_{l}\right)$ is called the free entropy of $a_{1}, \ldots, a_{n}$ in the presence of $b_{1}, \ldots, b_{l}$. Replacing the microstate spaces above with $\Gamma_{R}\left(a_{1}, \ldots, a_{n} ; m, k, \gamma\right)$ yields $\chi\left(a_{1}, \ldots, a_{n}\right)$, which is simply called the free entropy of $a_{1}, \ldots, a_{n}$. 
Now suppose $\left\{s_{1}, \ldots, s_{n}\right\}$ is a set of freely independent semicircular elements in $M$ (by this we mean that $\left\langle\left\{s_{i}\right\}\right\rangle_{i=1}^{n}$ is a family of freely independent sets such that for each $1 \leq j \leq n, s_{j}$ is selfadjoint and for any $\left.d \in \mathbb{N}, \varphi\left(s_{j}^{d}\right)=\frac{2}{\pi} \int_{-1}^{1} t^{d} \sqrt{1-t^{2}} d t\right)$ such that the von Neumann algebra they generate is freely independent with respect to the strongly closed algebra generated by $\left\{a_{1}, \ldots, a_{n}\right\}$. Define the modified free entropy dimension of $\left\{a_{1}, \ldots, a_{n}\right\}$ by

$$
\delta_{0}\left(a_{1}, \ldots, a_{n}\right)=n+\limsup _{\epsilon \rightarrow 0} \frac{\chi\left(a_{1}+\epsilon s_{1}, \ldots, a_{n}+\epsilon s_{n}: s_{1}, \ldots, s_{n}\right)}{|\log \epsilon|} .
$$

One can view the modified free entropy dimension as a noncommutative analogue of the Minkowski dimension. Given $S \subset \mathbb{R}^{d}$ the Minkowski dimension of $S$ is $d+\lim \sup _{\epsilon \rightarrow 0} \frac{\mu\left(N_{\epsilon}(S)\right)}{|\log \epsilon|}$ where $\mu$ is Lebesgue measure on $\mathbb{R}^{d}$ and $N_{\epsilon}(S)$ is the $\epsilon$ neighborhood of $S$ (technically this is the upper Minkowski dimension of $S$ ). The Minkowski dimension defined for $S$ turns out to be the same as the metric entropy quantity $\lim \sup _{\epsilon \rightarrow 0} \frac{\log P_{\epsilon}(S)}{|\log \epsilon|}$ where $P_{\epsilon}(S)$ is the maximum number of points in an $\epsilon$ separated subset of $S$ (the $\epsilon$ packing number of $S$ ). We will have more to say about the connections between Minkowski/metric entropy and free entropy dimension.

Here are a few basic properties of $\delta_{0}$, all of which are proven in [10]:

- For $1 \leq j \leq n, \delta_{0}\left(a_{1}, \ldots, a_{n}\right) \leq \delta_{0}\left(a_{1}, \ldots, a_{j}\right)+\delta_{0}\left(a_{j+1}, \ldots, a_{n}\right)$.

- For any $a=a^{*} \in M, \delta_{0}(a)=1-\sum_{t \in s p(a)}(\lambda(\{t\}))^{2}$ where $\lambda$ is the Borel measure on $s p(a)$ induced by $\varphi$.

- If $\chi\left(a_{1}, \ldots, a_{n}\right)>-\infty$, then $\delta_{0}\left(a_{1}, \ldots, a_{n}\right)=n$.

- If $a_{1}, \ldots, a_{n}$ are freely independent, then $\delta_{0}\left(a_{1}, \ldots, a_{n}\right)=\delta_{0}\left(a_{1}\right)+\ldots$ $+\delta_{0}\left(a_{n}\right)$.

Unfortunately, it is not known whether $\delta_{0}$ is an invariant of von Neumann algebras with tracial states, i.e., if $\left\{b_{1}, \ldots, b_{p}\right\}$ and $\left\{a_{1}, \ldots, a_{n}\right\}$ are two sets of selfadjoint generators for $M$ (this means that each set generates a strongly closed algebra equal to $M$ and each element of the set is selfadjoint), then does it follow that $\delta_{0}\left(a_{1}, \ldots, a_{n}\right)=\delta_{0}\left(b_{1}, \ldots, b_{p}\right)$ ? An affirmative answer to this question would show that for $m \neq n, L\left(F_{m}\right)$ is not $*$-isomorphic to $L\left(F_{n}\right)$, for it is well known that for any $m \in \mathbb{N}$ there exist $m$ semicircular generators $s_{1}, \ldots, s_{m}$ for $L\left(F_{m}\right)$ that satisfy $\delta_{0}\left(s_{1}, \ldots, s_{m}\right)=m$.

1.2. Results. We show that for hyperfinite von Neumann algebras with specified tracial state, $\delta_{0}$ is an invariant. More specifically, suppose $M$ is a hyperfinite von Neumann algebra with normal, tracial state $\varphi$. By decomposing $M$ over its center it follows that

$$
\begin{aligned}
& M \simeq M_{0} \oplus\left(\bigoplus_{i=1}^{s} M_{k_{i}}(\mathbb{C})\right) \oplus M_{\infty}, \\
& \varphi \simeq \alpha_{0} \varphi_{0} \oplus\left(\bigoplus_{i=1}^{s} \alpha_{i} t_{k_{i}}\right) \oplus 0
\end{aligned}
$$

where $s \in \mathbb{N} \cup\{0\} \cup\{\infty\}, \alpha_{i}>0$ for $1 \leq i \leq s(i \in \mathbb{N}), M_{0}$ is a diffuse von Neumann algebra or $\{0\}, \varphi_{0}$ is a faithful, tracial state on $M_{0}$ and $\alpha_{0}>0$ if $M_{0} \neq\{0\}, \varphi_{0}=0$ and $\alpha_{0}=0$ if $M_{0}=\{0\}$, and $M_{\infty}$ is a von Neumann algebra or $\{0\}$. We show that 
for any selfadjoint generators $a_{1}, \ldots, a_{n}$ for $M$,

$$
\delta_{0}\left(a_{1}, \ldots, a_{n}\right)=1-\sum_{i=1}^{s} \frac{\alpha_{i}^{2}}{k_{i}^{2}} .
$$

Because every such $M$ has a finite set of selfadjoint generators, it makes sense to define $\delta_{0}(M)=\delta_{0}\left(a_{1}, \ldots, a_{n}\right)$ where $\left\{a_{1}, \ldots, a_{n}\right\}$ is a set of selfadjoint generators for $M$. It follows that for any $k \in \mathbb{N}, \quad \delta_{0}\left(M_{k}(\mathbb{C})\right)=1-\frac{1}{k^{2}}$ and if $M$ is the hyperfinite $I I_{1}$-factor, then $\delta_{0}(M)=1$. The calculations also show that for hyperfinite $M$ the free entropy dimension number we obtain for $M$ coincides with the "free dimension" number for $M$ which appears in Dykema's work [2].

As a consequence of the arguments leading towards the above result we obtain a "hyperfinite monotonicity" property of $\delta_{0}$ which says the following. Suppose $M$ is an arbitrary von Neumann algebra with specified tracial state and selfadjoint generators $a_{1}, \ldots, a_{n}$. Assume moreover that $\left\{a_{1}, \ldots, a_{n}\right\}$ has finite-dimensional approximants, i.e., for any $m \in \mathbb{N}, \epsilon>0$, and $L>\max \left\{\left\|a_{i}\right\|\right\}_{1 \leq i \leq n}$ there exists an $N \in \mathbb{N}$ such that for all $k \geq N, \Gamma_{L}\left(a_{1}, \ldots, a_{n} ; m, k, \epsilon\right) \neq \emptyset$. If $N$ is a hyperfinite von Neumann subalgebra of $M$, then

$$
\delta_{0}(N) \leq \delta_{0}\left(a_{1}, \ldots, a_{n}\right) .
$$

Hyperfinite monotonicity of $\delta_{0}$ paired with a result by Voiculescu ([10]) show that if $M$ has a regular, diffuse, hyperfinite von Neumann subalgebra, then $\delta_{0}\left(a_{1}, \ldots, a_{n}\right)=$ 1 (see Remark 4.8).

The gist of the argument is simple: Essentially $\delta_{0}\left(a_{1}, \ldots, a_{n}\right)$ is the normalized metric entropy of the unitary orbit of a well-approximating microstate for $\left\{a_{1}, \ldots, a_{n}\right\}$. Suppose $M$ is hyperfinite with specified tracial state and selfadjoint generators $\left\{a_{1}, \ldots, a_{n}\right\} . \chi\left(a_{1}+\epsilon s_{1}, \ldots, a_{n}+\epsilon s_{n}: s_{1}, \ldots, s_{n}\right)$ is more or less the normalized logarithm of the volume of the $\epsilon$-neighborhood around the microstates of $\left\{a_{1}, \ldots, a_{n}\right\}$. $M$ being hyperfinite any two such microstates are approximately unitarily equivalent. So $\chi\left(a_{1}+\epsilon s_{1}, \ldots, a_{n}+\epsilon s_{n}: s_{1}, \ldots, s_{n}\right)$ is a limiting process calculated from $k^{-2}$ times the logarithm of the volume of the $\epsilon$-neighborhood of the unitary orbit of a single microstate for $\left\{a_{1}, \ldots, a_{n}\right\}$. Dividing this quantity by $|\log \epsilon|$ and adding $n$ is close to $k^{-2}$ multiplied by the Minkowski dimension of the unitary orbit of the microstate or, equivalently, the metric entropy of the set. Very roughly then, $\delta_{0}\left(a_{1}, \ldots, a_{n}\right)$ is the normalized metric entropy of the unitary orbit of a single well-approximating microstate for $\left\{a_{1}, \ldots, a_{n}\right\}$.

The calculations require more delicacy than we have let on, for we must first fix an $\epsilon$ and find the volume bounds/packing number bounds with respect to $\epsilon$ not merely over one microstate in one dimension but over one microstate for each dimension (because the first process in free entropy takes a limit as the dimensions go to infinity). Weak inequalities reduce this to either the investigation of uniform bounds on the packing numbers of homogeneous spaces obtained from $U_{k}$, the $k \times k$ unitaries, or to $\delta_{0}(a)$ where $a$ is a selfadjoint element. In the former case we make crucial use of the results of Szarek ([7]) and Raymond ([5]). The latter situation dealing with $\delta_{0}(a)$ has already been discussed.

We break up the paper into calculating upper and lower bounds for $\delta_{0}\left(a_{1}, \ldots, a_{n}\right)$ where $a_{1}, \ldots, a_{n}$ are arbitrary selfadjoint generators for hyperfinite $M$ with specified tracial state. Section 2 is a short list of notation and assumptions we make throughout the paper. Section 3 obtains the upper bound for general $M$. Section 
4 shows that if $\left\{a_{1}, \ldots, a_{n}\right\}$ is a set of selfadjoint generators of a diffuse (arbitrary, i.e., not necessarily hyperfinite) von Neumann algebra with a tracial state and $\left\{a_{1}, \ldots, a_{n}\right\}$ has finite-dimensional approximants, then $\delta_{0}\left(a_{1}, \ldots, a_{n}\right) \geq 1$. In particular, this yields the desired lower bound for diffuse $M$. Section 5 obtains the lower bound when $M$ is finite dimensional and Section 6 combines the results of Sections 4 and 5 to arrive at the general lower bound. Section 7 gleans immediate corollaries (including hyperfinite monotonicity of $\delta_{0}$ ) and comments on the relation of $\delta_{0}(M)$ to Dykema's free dimension [2]. Section 8 is an addendum where we prove some consequences of Szarek's metric entropy bounds of homogeneous spaces.

\section{Definitions and Notation}

Throughout this paper we maintain the notation in the introduction. Also, we assume throughout that $M$ is a von Neumann algebra (not necessarily hyperfinite) with separable predual, a unit $I$, and a normal, tracial state $\varphi .\left\{s_{i}: i \in \mathbb{N}\right\}$ is always a semicircular family free with respect to $M .\left\{a_{1}, \ldots, a_{n}\right\} \subset M$ is a set of selfadjoint generators for $M$ with finite-dimensional approximants. $R=\max \left\{\left\|a_{i}\right\|\right\}_{1 \leq i \leq n}$. Lastly, $|\cdot|_{2}$ is the norm on $M_{k}(\mathbb{C})$ or the seminorm on $M$ given by $|x|_{2}=\left(\operatorname{tr}_{k}\left(x^{*} x\right)\right)^{\frac{1}{2}}$ or $|x|_{2}=\left(\varphi\left(x^{*} x\right)\right)^{\frac{1}{2}}$, respectively.

\section{UpPer Bound}

Throughout the section assume that $M$ is hyperfinite and that $N$ is a finitedimensional $*$-subalgebra of $M$ containing $I$. Also assume that $N$ has selfadjoint generators $\left\{b_{1}, \ldots, b_{n}\right\}$ such that each $b_{i}$ has operator norm no larger than $R$. For any $k \in \mathbb{N}$ and $\epsilon>0, T\left(b_{1}, \ldots, b_{n} ; k, \epsilon\right)$ denotes

$$
\begin{gathered}
\left\{\left(x_{1}, \ldots, x_{n}\right) \in\left(M_{k}^{s a}(\mathbb{C})\right)^{n}: \text { there exists a } * \text {-homomorphism } \sigma: N \rightarrow M_{k}(\mathbb{C})\right. \\
\text { such that for all } \left.1 \leq i \leq n, \quad\left|\sigma\left(b_{i}\right)-x_{i}\right|_{2} \leq \epsilon \text { and }\left\|t r_{k} \circ \sigma-\left.\varphi\right|_{N}\right\|<\epsilon^{2}\right\}
\end{gathered}
$$

We show that $\delta_{0}\left(a_{1}, \ldots, a_{n}\right) \leq 1-\sum_{i=1}^{s} \frac{\alpha_{i}^{2}}{k_{i}^{2}}$ where the $\alpha_{i}$ and $k_{i}$ are as in the canonical decomposition of $M$ discussed on page 3 of the introduction. The argument proceeds in several easy steps. First, $\chi\left(a_{1}+\epsilon s_{1}, \ldots, a_{n}+\epsilon s_{n}: s_{1}, \ldots, s_{n}\right)$ is dominated by a number calculated more or less from $\operatorname{vol}\left(T\left(b_{1}, \ldots, b_{n} ; k, \epsilon\right)\right)$. Secondly, $T\left(b_{1}, \ldots, b_{n} ; k, \epsilon\right)$ is contained in the neighborhood of a restricted unitary orbit of any single element in $T\left(b_{1}, \ldots, b_{n}: k, \epsilon\right)$. Szarek's packing number estimates provide appropriate upper bounds for the volume of the neighborhoods of such orbits. Finally, by approximating $M$ by fine enough finite-dimensional *subalgebras $N$ of $M$, standard approximation arguments yield the promised upper bound.

The first lemma presented below is standard and we omit the proof. It amounts to saying that matricial microstates for selfadjoint generators of a finite-dimensional von Neumann algebra correspond to approximate representations.

Lemma 3.1. For each $\epsilon>0$ there exist an $m \in \mathbb{N}$ and $\gamma>0$ such that for all $k \in \mathbb{N}$,

$$
\Gamma_{R+1}\left(b_{1}, \ldots, b_{n}: m, k, \gamma\right) \subset T\left(b_{1}, \ldots, b_{n} ; k, \epsilon\right)
$$


Lemma 3.1 has a trivial consequence:

Lemma 3.2. Suppose $\epsilon_{0}>0$ and $\max \left\{\left|a_{i}-b_{i}\right|_{2}: 1 \leq i \leq n\right\}<\epsilon_{0}$. For any $1>\epsilon>0, \chi\left(a_{1}+\epsilon s_{1}, \ldots, a_{n}+\epsilon s_{n}: s_{1}, \ldots, s_{n}\right)$ is dominated by

$$
\limsup _{k \rightarrow \infty}\left[k^{-2} \cdot \log \left(\operatorname{vol}\left(T\left(b_{1}, \ldots, b_{n} ; k, 3 \epsilon+\epsilon_{0}\right)\right)\right)+\frac{n}{2} \cdot \log k\right] .
$$

Proof. By Lemma 3.1 for a given $\epsilon \in(0,1)$ there exist $2 \leq m \in \mathbb{N}$ and $\gamma>0$ such that for any $k \in \mathbb{N}, \Gamma_{R+1}\left(b_{1}, \ldots, b_{n} ; m, k, \gamma\right) \subset T\left(b_{1}, \ldots, b_{n} ; k, \epsilon\right)$. For $\gamma^{\prime}>0$ if

$$
\left(x_{1}, \ldots, x_{n}\right) \in \Gamma_{R+1}\left(a_{1}+\epsilon s_{1}, \ldots, a_{n}+\epsilon s_{n}: b_{1}, \ldots, b_{n}, s_{1}, \ldots, s_{n} ; m, k, \gamma^{\prime}\right),
$$

then by definition there exists $\left(y_{1}, \ldots, y_{n}, z_{1}, \ldots, z_{n}\right) \in\left(M_{k}^{s a}(\mathbb{C})\right)^{2 n}$ satisfying

$$
\begin{aligned}
& \left(x_{1}, \ldots, x_{n}, y_{1}, \ldots, y_{n}, z_{1}, \ldots, z_{n}\right) \\
& \quad \in \Gamma_{R+1}\left(a_{1}+\epsilon s_{1}, \ldots, a_{n}+\epsilon s_{n}, b_{1}, \ldots, b_{n}, s_{1}, \ldots, s_{n} ; m, k, \gamma^{\prime}\right) .
\end{aligned}
$$

By choosing $\gamma^{\prime}<\gamma$ sufficiently small one can force $\left|x_{i}-y_{i}\right|_{2}<2 \epsilon+\epsilon_{0}$ for $1 \leq$ $i \leq n$. Since $\gamma^{\prime}<\gamma,\left(y_{1}, \ldots, y_{n}\right) \in \Gamma_{R+1}\left(b_{1}, \ldots, b_{n} ; m, k, \gamma\right) \subset T\left(b_{1}, \ldots, b_{n} ; k, \epsilon\right)$. Consequently, $\left(x_{1}, \ldots, x_{n}\right) \in T\left(b_{1}, \ldots, b_{n} ; k, 3 \epsilon+\epsilon_{0}\right)$. We have just shown that

$\Gamma_{R+1}\left(a_{1}+\epsilon s_{1}, \ldots, a_{n}+\epsilon s_{n}: b_{1}, \ldots, b_{n}, s_{1}, \ldots, s_{n} ; m, k, \gamma^{\prime}\right) \subset T\left(b_{1}, \ldots, b_{n} ; k, 3 \epsilon+\epsilon_{0}\right)$.

Basic properties of free entropy imply that $\chi\left(a_{1}+\epsilon s_{1}, \ldots, a_{n}+\epsilon s_{n}: s_{1}, \ldots, s_{n}\right)$ equals

$$
\begin{aligned}
& \chi_{R+1}\left(a_{1}+\epsilon s_{1}, \ldots, a_{n}+\epsilon s_{n}: b_{1}, \ldots, b_{n}, s_{1}, \ldots, s_{n}\right) \\
& \quad \leq \chi_{R+1}\left(a_{1}+\epsilon s_{1}, \ldots, a_{n}+\epsilon s_{n}: b_{1}, \ldots, b_{n}, s_{1}, \ldots, s_{n} ; m, \gamma^{\prime}\right) .
\end{aligned}
$$

By the preceding inclusion the dominating term is less than or equal to

$$
\limsup _{k \rightarrow \infty}\left[k^{-2} \cdot \log \left(\operatorname{vol}\left(T\left(b_{1}, \ldots, b_{n} ; k, 3 \epsilon+\epsilon_{0}\right)\right)\right)+\frac{n}{2} \cdot \log k\right] .
$$

Now for the claim which implies that $T\left(b_{1}, \ldots, b_{n} ; k, \epsilon\right)$ is contained in the $2 \epsilon(1+\sqrt{2} R)$ - neighborhood of a restricted unitary orbit of any single element of $T\left(b_{1}, \ldots, b_{n} ; k, \epsilon\right)$.

Because $\mathrm{N}$ is finite dimensional and $\varphi$ is tracial, assume from now on that $N \simeq$ $\bigoplus_{j=1}^{p} M_{n_{j}}(\mathbb{C})$ and $\left.\varphi\right|_{N} \simeq \bigoplus_{j=1}^{p} \alpha_{j} t r_{n_{j}}$.

Lemma 3.3. For any $k \in \mathbb{N}$ and $\epsilon>0$, if $\sigma_{1}, \sigma_{2}: N \rightarrow M_{k}(\mathbb{C})$ are $*$-homomorphisms such that $\left\|t r_{k} \circ \sigma_{1}-t r_{k} \circ \sigma_{2}\right\| \leq \epsilon^{2}$, then there exists a $u \in U_{k}$ such that

$$
\left|u\left(\sigma_{1}(x)\right) u^{*}-\sigma_{2}(x)\right|_{2} \leq 2\|x\| \epsilon .
$$

Proof. Without loss of generality, assume that $N=M_{n_{1}}(\mathbb{C}) \oplus \cdots \oplus M_{n_{p}}(\mathbb{C})$. For any $l_{1}, \ldots, l_{p} \in \mathbb{N} \cup\{0\}$ with $\sum_{i=1}^{p} n_{i} l_{i} \leq k$ denote by $\pi_{l_{1}, \ldots, l_{p}}: N \rightarrow M_{k}(\mathbb{C})$ the *-homomorphism

$$
\pi_{l_{1}, \ldots, l_{p}}\left(x_{1}, \ldots, x_{p}\right)=\left[\begin{array}{cccc}
x_{1} \otimes I_{l_{1}} & 0 & \ldots & 0 \\
0 & \ddots & & \vdots \\
\vdots & & x_{p} \otimes I_{l_{p}} & 0 \\
0 & \ldots & 0 & 0_{p+1}
\end{array}\right]
$$


where $x_{i} \bigotimes I_{l_{i}}$ is the $n_{i} l_{i} \times n_{i} l_{i}$ matrix with $x_{i}$ repeated $l_{i}$ times on the diagonal and $0_{p+1}$ is the $\left(k-\sum_{i=1}^{p} n_{i} l_{i}\right) \times\left(k-\sum_{i=1}^{p} n_{i} l_{i}\right) 0$ matrix. There exist $m_{1}, \ldots, m_{p}, n_{1}, \ldots, n_{p} \in \mathbb{N} \cup\{0\}$ such that $\sigma_{1} \sim \pi_{m_{1}, \ldots, m_{p}}, \sigma_{2} \sim \pi_{n_{1}, \ldots, n_{p}}$.

Set $d_{i}=\min \left\{m_{i}, n_{i}\right\}$ and observe that if $\pi=\pi_{d_{1}, \ldots, d_{p}}$, then $\left\|t r_{k} \circ \pi-t r_{k} \circ \sigma_{j}\right\| \leq \epsilon^{2}$ for $j=1,2$. If we can show that for each $j$ there exists a $u_{j} \in U_{k}$ satisfying $\left|u_{j}\left(\sigma_{j}(x)\right) u_{j}^{*}-\pi(x)\right|_{2} \leq\|x\| \epsilon$, then we will be done. A moment's thought shows that for each $j$ there exists a $u_{j} \in U_{k}$ such that $\operatorname{Ad} u_{j} \circ \sigma_{j}-\pi$ is a $*$-homomorphism which we will denote by $\rho_{j}$. Obviously $\left\|t r_{k} \circ \rho_{j}\right\| \leq \epsilon^{2}$ so that for any $x \in N$,

$$
\left|u_{j}\left(\sigma_{j}(x)\right) u_{j}^{*}-\pi(x)\right|_{2}=\left(\left(\operatorname{tr}_{k} \circ \rho_{j}\right)\left(x^{*} x\right)\right)^{1 / 2} \leq\left(\epsilon^{2} \cdot\left\|x^{*} x\right\|\right)^{1 / 2}=\|x\| \epsilon .
$$

Given $\left(x_{1}, \ldots, x_{n}\right),\left(y_{1}, \ldots, y_{n}\right) \in T\left(b_{1}, \ldots, b_{n} ; k, \epsilon\right)$ there are representations $\sigma$, $\pi: N \rightarrow M_{k}(\mathbb{C})$ such that for $1 \leq i \leq n,\left|\sigma\left(b_{i}\right)-x_{i}\right|_{2},\left|\pi\left(b_{i}\right)-y_{i}\right|_{2}<\epsilon$ and $\left\|t r_{k} \circ \sigma-\left.\varphi\right|_{N}\right\|,\left\|t r_{k} \circ \pi-\left.\varphi\right|_{N}\right\|<\epsilon^{2}$. So $\left\|t r_{k} \circ \sigma-t r_{k} \circ \pi\right\|<2 \epsilon^{2}$. By Lemma 3.3 there exists a $u \in U_{k}$ satisfying

$$
\left|u\left(\sigma\left(b_{i}\right)\right) u^{*}-\pi\left(b_{i}\right)\right|_{2} \leq 2\left\|b_{i}\right\|(\sqrt{2} \epsilon) \leq 2 \sqrt{2} R \epsilon
$$

for $1 \leq i \leq n$. Thus $\left|u x_{i} u^{*}-y_{i}\right|_{2} \leq 2 \epsilon+2 \sqrt{2} R \epsilon=2 \epsilon(1+\sqrt{2} R)$. From now on for $z \in M_{k}^{s a}(\overline{\mathbb{C}})$ and $\gamma>0$ define $B(z, \gamma)=\left\{x \in M_{k}^{s a}(\mathbb{C}):|x-z|_{2}<\gamma\right\}$. We have just proved:

Corollary 3.4. If $\left(x_{1}, \ldots, x_{n}\right) \in T\left(b_{1}, \ldots, b_{n} ; k, \epsilon\right)$, then $T\left(b_{1}, \ldots, b_{n} ; k, \epsilon\right)$ is contained in

$$
\bigcup_{u \in U_{k}}\left[B\left(u x_{1} u^{*}, 2 \epsilon(1+\sqrt{2} R)\right) \times \cdots \times B\left(u x_{n} u^{*}, 2 \epsilon(1+\sqrt{2} R)\right)\right] .
$$

We remark here that Lemma 3.1, Lemma 3.3, and Corollary 3.4 also hold in the situation where $N$ does not contain $I$.

We now draw out a trivial consequence of Szarek's estimates for covering numbers of homogeneous spaces. These results are the heart of the calculation of the upper bound.

For $k \in \mathbb{N}$ suppose $m, k_{1}, \ldots, k_{m}, l_{1}, \ldots, l_{m} \in \mathbb{N}, \sum_{i=1}^{m} k_{i} l_{i}=k$, and $H \subset U_{k}$ is a proper Lie subgroup of $U_{k}$ consisting of all matrices of the form

$$
\left[\begin{array}{ccc}
u_{1} \otimes I_{k_{1}} & \cdots & 0 \\
\vdots & \ddots & \vdots \\
0 & \cdots & u_{m} \otimes I_{k_{m}}
\end{array}\right]
$$

where $u_{i} \in U_{l_{i}}$ and $u_{i} \otimes I_{k_{i}}$ is the $k_{i} l_{i} \times k_{i} l_{i}$ matrix obtained by repeating $u_{i} k_{i}$ times along the diagonal. Such Lie subgroups $H$ of $U_{k}$ will be called tractable.

A simple application of Theorem 11 in [7] yields:

Lemma 3.5. There exist constants $C, \beta>0$ such that for any $k \in \mathbb{N}$, any tractable Lie subgroup $H$ of $U_{k}$ and $\epsilon \in(0, \beta)$,

$$
N(X, \epsilon) \leq\left(\frac{C}{\epsilon}\right)^{d}
$$

where $X$ is the manifold $U_{k} / H$ endowed with the quotient metric induced by the operator norm, $d$ is the real dimension of $X$, and $N(X, \epsilon)$ is the minimum number of balls of radius $\epsilon$ required to cover $X$. 
We sequester a rigorous demonstration to the Addendum.

Lemma 3.6. If $1>r>0$, then there exists a $k_{0} \in \mathbb{N}$ such that for each $k>k_{0}$ there is a corresponding $*$-homomorphism $\sigma_{k}: N \rightarrow M_{k}(\mathbb{C})$ satisfying:

- $\left\|t r_{k} \circ \sigma_{k}-\left.\varphi\right|_{N}\right\|<r^{2}$.

- The unitaries $H_{k}$ of $\sigma_{k}(N)^{\prime}$ form a tractable Lie subgroup of $U_{k}$ satisfying

$$
k^{2}\left(1-r-\sum_{i=1}^{p} \frac{\alpha_{i}^{2}}{n_{i}^{2}}\right)<\operatorname{dim}\left(U_{k} / H_{k}\right)<k^{2}\left(1+r-\sum_{i=1}^{p} \frac{\alpha_{i}^{2}}{n_{i}^{2}}\right) .
$$

Proof. Suppose that $1>\varepsilon>0$. Choose $n_{0} \in \mathbb{N}$ such that $\frac{1}{n_{0}}<\frac{\varepsilon}{p+1}$. Set $k_{0}=$ $\left(n_{0}+1\right) n_{1} \cdots n_{p}$. Suppose $k>k_{0}$. Find the unique $n \in \mathbb{N}$ (dependent on $k$ ) satisfying

$$
n n_{1} \cdots n_{p} \leq k<(n+1) n_{1} \cdots n_{p} .
$$

Set $d=n n_{1} \cdots n_{p}\left(d\right.$ dependent on $k$ ) and find $m_{1}, \ldots, m_{p} \in \mathbb{N} \cup\{0\}$ satisfying $\alpha_{i}-\varepsilon<\frac{m_{i}}{n}<\alpha_{i}+\varepsilon$ for each $i$ and $\sum_{i=1}^{p} \frac{m_{i}}{n}=1$ (the $m_{i}$ depend on $k$ ). Set $l_{i}(k)=\frac{d m_{i}}{n n_{i}} \in \mathbb{N} \cup\{0\}$ and $l_{p+1}(k)=k-\sum_{i=1}^{p} l_{i}(k) n_{i}$. Assume without loss of generality that $N=M_{n_{1}}(\mathbb{C}) \oplus \cdots \oplus M_{n_{p}}(\mathbb{C})$ and $\left.\varphi\right|_{N}=\alpha_{1} t r_{n_{1}} \oplus \cdots \oplus \alpha_{p} t r_{n_{p}}$. Define $\sigma_{k}: N \rightarrow M_{k}(\mathbb{C})$ by

$$
\sigma_{k}\left(x_{1}, \ldots, x_{n}\right)=\left[\begin{array}{cccc}
I_{l_{1}(k)} \otimes x_{1} & 0 & \ldots & 0 \\
0 & \ddots & & \vdots \\
\vdots & & I_{l_{p}(k)} \otimes x_{p} & 0 \\
0 & \ldots & 0 & 0_{l_{p+1}(k)}
\end{array}\right]
$$

where $0_{l_{p+1}(k)}$ is the $l_{p+1}(k) \times l_{p+1}(k) 0$ matrix and $I_{l_{i}(k)} \otimes x_{i}$ is the $l_{i}(k) n_{i} \times l_{i}(k) n_{i}$ matrix obtained by taking each entry of $x_{i},\left(x_{i}\right)_{s t}$, and stretching it out into $\left(x_{i}\right)_{s t}$. $I_{l_{i}(k)}$ where $I_{l_{i}(k)}$ is the $l_{i}(k) \times l_{i}(k)$ identity matrix. Then

$$
\left(t r_{k} \circ \sigma\right)\left(x_{1}, \ldots, x_{p}\right)=\frac{1}{k} \cdot \sum_{i=1}^{p} l_{i}(k) \cdot \operatorname{Tr}\left(x_{i}\right)=\sum_{i=1}^{p} \frac{d m_{i}}{k n} \cdot t r_{n_{i}}\left(x_{i}\right) .
$$

Since $\frac{d}{k}>1-\varepsilon$, we have $\alpha_{i}+\varepsilon \geq \frac{d}{k} \cdot \frac{m_{i}}{n}>\left(\alpha_{i}-\varepsilon\right)(1-\varepsilon)>\alpha_{i}-2 \varepsilon$. It follows that $\left\|t r_{k} \circ \sigma_{k}-\left.\varphi\right|_{N}\right\|<2 p \varepsilon$.

$H_{k}$ consists of all matrices of the form

$$
\left[\begin{array}{cccc}
u_{1} \otimes I_{n_{1}} & 0 & \cdots & 0 \\
0 & \ddots & & \vdots \\
\vdots & & u_{p} \otimes I_{n_{p}} & 0 \\
0 & \cdots & 0 & u_{p+1}
\end{array}\right]
$$

where $u_{i} \in U_{l_{i}(k)}$ for $1 \leq i \leq p+1$ and $u_{i} \otimes I_{n_{i}}$ is the $l_{i}(k) n_{i} \times l_{i}(k) n_{i}$ matrix obtained by repeating $u_{i} n_{i}$ times along the diagonal. $H_{k}$ is obviously tractable.

For a lower bound on $\operatorname{dim}\left(U_{k} / H_{k}\right)$ we have the estimate:

$$
l_{p+1}(k)=k-\sum_{i=1}^{p} \frac{d m_{i}}{n}=k-d<n_{1} \cdots n_{p}<\frac{k_{0}}{n_{0}}<k \cdot \varepsilon
$$


so that

$$
\operatorname{dim} H_{k}=l_{p+1}(k)^{2}+\sum_{i=1}^{p} l_{i}(k)^{2}<k^{2}\left(\varepsilon+\sum_{i=1}^{p} \frac{\left(\alpha_{i}+\varepsilon\right)^{2}}{n_{i}^{2}}\right)<k^{2}\left(3 p \varepsilon+\sum_{i=1}^{p} \frac{\alpha_{i}^{2}}{n_{i}^{2}}\right) .
$$

Hence, $\operatorname{dim}\left(U_{k} / H_{k}\right)=k^{2}-\operatorname{dim} H_{k}$ is bounded from below by $k^{2}\left(1-3 p \varepsilon-\sum_{i=1}^{p} \frac{\alpha_{i}^{2}}{n_{i}^{2}}\right)$.

For an upper bound on $\operatorname{dim}\left(U_{k} / H_{k}\right)$ observe that $\operatorname{dim} H_{k}>\sum_{i=1}^{p} l_{i}(k)^{2}$ whence

$$
\begin{aligned}
\operatorname{dim}\left(U_{k} / H_{k}\right)=k^{2}-\operatorname{dim} H_{k} & <k^{2}-\sum_{i=1}^{p} l_{i}(k)^{2} \\
& <k^{2}\left(1-\sum_{i=1}^{p} \frac{\left(\alpha_{i}-2 \varepsilon\right)^{2}}{n_{i}^{2}}\right) \\
& <k^{2}\left(1+4 \varepsilon-\sum_{i=1}^{p} \frac{\alpha_{i}^{2}}{n_{i}^{2}}\right) .
\end{aligned}
$$

Set $\varepsilon=\frac{r^{2}}{4 p}$.

We now make the key calculation on the upper bound of Lemma 3.2.

Lemma 3.7. For $\min \{\beta, C\}>\epsilon>0$,

$$
\limsup _{k \rightarrow \infty}\left[k^{-2} \cdot \log \left(\operatorname{vol}\left(T\left(b_{1}, \ldots, b_{n} ; k, \epsilon\right)\right)\right)+\frac{n}{2} \cdot \log k\right] \leq \log \left(\epsilon^{n-\triangle}\right)+\log D
$$

where $\triangle=1-\sum_{i=1}^{p} \frac{\alpha_{i}^{2}}{n_{i}^{2}}$ and $D=\pi^{\frac{n}{2}}(8(R+1))^{n} C^{\triangle}\left[(2 e)^{\frac{n}{2}}\right]$.

Proof. Suppose $\min \{\beta, C\}>\epsilon>r>0$. By Lemma 3.6 there is a $k_{0} \in \mathbb{N}$ such that for each $k \geq k_{0}$ there exists a $*$-homomorphism $\sigma_{k}: N \rightarrow M_{k}(\mathbb{C})$ satisfying $\left\|t r_{k} \circ \sigma_{k}-\left.\varphi\right|_{N}\right\|<r^{2}$ and the additional condition that if $H_{k}$ is the unitary group of $\sigma_{k}(N)^{\prime}$, then $H_{k}$ is tractable and

$$
\operatorname{dim}\left(U_{k} / H_{k}\right)<k^{2}\left(1+r-\sum_{i=1}^{p} \frac{\alpha_{i}^{2}}{n_{i}^{2}}\right) .
$$

Set

$$
d_{k}=\operatorname{dim}\left(U_{k} / H_{k}\right) \quad \text { and } \quad m_{r}=-r+\sum_{i=1}^{p} \frac{\alpha_{i}^{2}}{n_{i}^{2}} .
$$

There exists a set $\left\langle u_{k, s}\right\rangle_{s \in S_{k}}$ contained in $U_{k}$ such that for each $u \in U_{k}$ there exists an $s \in S_{k}$ and $h \in H_{k}$ satisfying $\left\|u-u_{k, s} h\right\|<\epsilon$ and $S_{k}$ has cardinality not exceeding $\left(\frac{C}{\epsilon}\right)^{d_{k}}<\left(\frac{C}{\epsilon}\right)^{k^{2}\left(1-m_{r}\right)}$.

Set $L=8(R+1)$. I claim that

$$
T\left(b_{1}, \ldots, b_{n} ; k, \epsilon\right) \subset \bigcup_{s \in S_{k}}\left[B\left(u_{k, s} \sigma_{k}\left(b_{1}\right) u_{k, s}^{*}, L \epsilon\right) \times \cdots \times B\left(u_{k, s} \sigma_{k}\left(b_{n}\right) u_{k, s}^{*}, L \epsilon\right)\right] .
$$

Suppose that $\left(x_{1}, \ldots, x_{n}\right) \in T\left(b_{1}, \ldots, b_{n} ; k, \epsilon\right)$. Clearly $\left(\sigma_{k}\left(b_{1}\right), \ldots, \sigma_{k}\left(b_{n}\right)\right) \in$ $T\left(b_{1}, \ldots, b_{n} ; k, \epsilon\right)$. By Corollary 3.4 there exists a $u \in U_{k}$ such that for all $1 \leq$ 
$i \leq n, \quad\left|x_{i}-u \sigma_{k}\left(b_{i}\right) u^{*}\right|_{2} \leq 2 \epsilon(1+\sqrt{2} R)$. There exists an $s \in S_{k}$ and $h \in H_{k}$ such that $\left\|u-u_{k, s} h\right\|<\epsilon$. Now

$$
\begin{aligned}
\left|u \sigma_{k}\left(b_{i}\right) u^{*}-u_{k, s} \sigma_{k}\left(b_{i}\right) u_{k, s}^{*}\right|_{2}= & \left|u \sigma_{k}\left(b_{i}\right) u^{*}-u_{k, s} h \sigma_{k}\left(b_{i}\right) h^{*} u_{k, s}^{*}\right|_{2} \\
\leq & \left\|u-u_{k, s} h\right\| \cdot\left|\sigma_{k}\left(b_{i}\right) u^{*}\right|_{2} \\
& +\left|u_{k, s} h \sigma_{k}\left(b_{i}\right)\right|_{2} \cdot\left\|u^{*}-h^{*} u_{k, s}^{*}\right\| \\
\leq & 2 \epsilon R .
\end{aligned}
$$

Hence for all $1 \leq i \leq n, \quad\left|x_{i}-u_{k, s} \sigma_{k}\left(b_{i}\right) u_{k, s}^{*}\right|_{2} \leq 2 \epsilon(1+\sqrt{2} R)+2 \epsilon R<L \epsilon$.

By the inclusion demonstrated in the preceding paragraph we have that $\log \left(\operatorname{vol}\left(T\left(b_{1}, \ldots, b_{n} ; k, \epsilon\right)\right)\right)$ is dominated by

$$
\begin{aligned}
\log \left(\left|S_{k}\right|\right. & \left.\cdot \frac{\pi^{\frac{n k^{2}}{2}}(L \sqrt{k} \epsilon)^{n k^{2}}}{\left(\Gamma\left(\frac{k^{2}}{2}+1\right)\right)^{n}}\right)=\log \left(\left|S_{k}\right| \cdot \frac{\left(\pi^{\frac{n}{2}} L^{n} k^{\frac{n}{2}} \epsilon^{n}\right)^{k^{2}}}{\left(\Gamma\left(\frac{k^{2}}{2}+1\right)\right)^{n}}\right) \\
& \leq k^{2} \cdot \log \left[\left(\frac{C}{\epsilon}\right)^{1-m_{r}}\left(\pi^{\frac{n}{2}} L^{n} k^{\frac{n}{2}} \epsilon^{n}\right)\right]-n \cdot \log \Gamma\left(\frac{k^{2}}{2}+1\right) \\
& \leq k^{2} \cdot \log \left(\pi^{\frac{n}{2}} L^{n} C^{1-m_{r}} k^{\frac{n}{2}} \epsilon^{n-1+m_{r}}\right)-n \cdot \log \left[\left(\frac{k^{2}}{2 e}\right)^{\frac{k^{2}}{2}}\right] \\
& =k^{2} \cdot \log \left(\pi^{\frac{n}{2}} L^{n} C^{1-m_{r}} \epsilon^{n-1+m_{r}}\right)-\frac{n k^{2}}{2} \cdot \log k+k^{2} \log \left[(2 e)^{\frac{n}{2}}\right]
\end{aligned}
$$

provided $k \geq k_{0} . \lim \sup _{k \rightarrow \infty}\left[k^{-2} \log \left(\operatorname{vol}\left(T\left(b_{1}, \ldots, b_{n} ; k, \epsilon\right)\right)\right)+\frac{n}{2} \cdot \log k\right]$ is therefore dominated by

$$
\begin{aligned}
\limsup _{k \rightarrow \infty} & \left(\log \left(\pi^{\frac{n}{2}} L^{n} C^{1-m_{r}} \epsilon^{n-1+m_{r}}\right)+\log \left[(2 e)^{\frac{n}{2}}\right]\right. \\
= & \log \left(\epsilon^{n-1+m_{r}}\right)+\log \left(\pi^{\frac{n}{2}} L^{n} C^{1-m_{r}}\left[(2 e)^{\frac{n}{2}}\right]\right) .
\end{aligned}
$$

Hence

$$
\begin{aligned}
\log \left(\epsilon^{n-\triangle}\right)+\log D & =\log \left(\epsilon^{n-\triangle}\right)+\log \left(\pi^{\frac{n}{2}} L^{n} C^{\triangle}\left[(2 e)^{\frac{n}{2}}\right]\right) \\
& =\lim _{r \rightarrow 0}\left[\left(\log \left(\epsilon^{n-1+m_{r}}\right)+\log \left(\pi^{\frac{n}{2}} L^{n} C^{1-m_{r}}\left[(2 e)^{\frac{n}{2}}\right]\right)\right]\right. \\
& \geq \limsup _{k \rightarrow \infty}\left[k^{-2} \cdot \log \left(\operatorname{vol}\left(T\left(b_{1}, \ldots, b_{n} ; k, \epsilon\right)\right)\right)+\frac{n}{2} \cdot \log k\right] .
\end{aligned}
$$

If $M$ is finite dimensional, then Lemma 3.7 yields the desired upper bound for $\delta_{0}\left(a_{1}, \ldots, a_{n}\right)$. With just a few more easy observations, Lemma 3.7 allows us to bootstrap the upper bound for $\delta_{0}\left(a_{1}, \ldots, a_{m}\right)$ in the general situation.

If $B$ is a finite-dimensional von Neumann algebra with a positive trace $\psi$ and $B \simeq \bigoplus_{i=1}^{s} M_{q_{i}}(\mathbb{C}), \psi \simeq \bigoplus_{i=1}^{s} r_{i} t r_{q_{i}}$, define $\triangle_{\psi}(B)=1-\sum_{i=1}^{s} \frac{r_{i}^{2}}{q_{i}^{2}}$. Clearly $\triangle_{\psi}(B)$ is well defined.

Lemma 3.8. If $A \subset B$ is a unital inclusion of finite-dimensional von Neumann algebras, and $\psi$ is a positive trace on $B$, then $\triangle_{\psi}(A) \leq \triangle_{\psi}(B)$.

Proof. By assumption $B \simeq \bigoplus_{j=1}^{s} M_{q_{j}}(\mathbb{C})$ and $\psi \simeq \bigoplus_{j=1}^{s} r_{j} t r_{q_{j}}$ for some $s, q_{1}, \ldots$, $q_{s}, r_{1}, \ldots, r_{s} \in \mathbb{N}$. $A$ is $*$-isomorphic to $\bigoplus_{i=1}^{d} M_{p_{i}}(\mathbb{C})$ for some $d, p_{1}, \ldots, p_{d} \in \mathbb{N}$. Denote $\left\langle\Lambda_{i j}\right\rangle_{1 \leq i \leq d, 1 \leq j \leq s}$ to be the inclusion matrix of $A$ into $B$ with respect to the 
dimension vectors $\left\langle p_{i}\right\rangle_{i=1}^{d}$ and $\left\langle q_{j}\right\rangle_{j=1}^{s}$ for $A$ and $B$, respectively. Since $A \subset B$ is a unital inclusion,

$$
\begin{gathered}
\sum_{i=1}^{d} \sum_{j=1}^{s} \frac{\left(\Lambda_{i j} p_{i} r_{j}\right)^{2}}{q_{j}^{2}} \cdot \frac{1}{p_{i}^{2}}=\sum_{j=1}^{s} \sum_{i=1}^{d} \frac{\Lambda_{i j}^{2} r_{j}^{2}}{q_{j}^{2}} \geq \sum_{j=1}^{s} \frac{r_{j}^{2}}{q_{j}^{2}} \\
\triangle_{\psi}(A)=1-\sum_{i=1}^{d}\left(\left(\sum_{j=1}^{s} \frac{\Lambda_{i j} p_{i} r_{j}}{q_{j}}\right)^{2} \cdot \frac{1}{p_{i}^{2}}\right) \leq 1-\sum_{j=1}^{s} \frac{r_{j}^{2}}{q_{j}^{2}}=\triangle_{\psi}(B) .
\end{gathered}
$$

Lemma 3.9. $\delta_{0}\left(a_{1}, \ldots, a_{n}\right)=\delta_{0}\left(a_{1}, \ldots, a_{n}, I\right)$.

Proof. By Propositions 6.4 and 6.6 of [10] and Proposition 6.3 of [9],

$$
\delta_{0}\left(a_{1}, \ldots, a_{n}, I\right) \leq \delta_{0}\left(a_{1}, \ldots, a_{n}\right)+\delta_{0}(I)=\delta_{0}\left(a_{1}, \ldots, a_{n}\right) .
$$

On the other hand, since the strongly closed $*$-algebra generated by $\left\{a_{1}, \ldots, a_{n}\right\}$ is $M$, by Theorem 4.3 of [10], $\delta_{0}\left(a_{1}, \ldots, a_{n}\right) \leq \delta_{0}\left(a_{1}, \ldots, a_{n}, I\right)$.

We are now in a position to calculate the upper bound for $\delta_{0}\left(a_{1}, \ldots, a_{n}\right)$. By decomposing $M$ over its center it follows that

$$
M \simeq M_{0} \oplus\left(\bigoplus_{i=1}^{s} M_{k_{i}}(\mathbb{C})\right) \oplus M_{\infty}, \quad \varphi \simeq \alpha_{0} \varphi_{0} \oplus\left(\bigoplus_{i=1}^{s} \alpha_{i} t r_{k_{i}}\right) \oplus 0
$$

where all quantities above are as in the introduction. Write $I_{i}$ for the identity of $M_{k_{i}}(\mathbb{C})$ for $1 \leq i \leq s$ and if $M_{0} \neq\{0\}$, then write $I_{0}$ for the identity of $M_{0}$. A moment's thought shows that for the purposes of the theorem below we can neglect the $M_{\infty}$ summand and assume

$$
M=M_{0} \oplus\left(\bigoplus_{i=1}^{s} M_{k_{i}}(\mathbb{C})\right), \quad \varphi=\alpha_{0} \varphi_{0} \oplus\left(\bigoplus_{i=1}^{s} \alpha_{i} t r_{k_{i}}\right) .
$$

Theorem 3.10. $\delta_{0}\left(a_{1}, \ldots, a_{n}\right) \leq 1-\sum_{i=1}^{s} \frac{\alpha_{i}^{2}}{k_{i}^{2}}$.

Proof. Set $\alpha=1-\sum_{i=1}^{s} \frac{\alpha_{i}^{2}}{k_{i}^{2}}$. There exists a nested sequence of finite-dimensional *-subalgebras of $M,\left\langle N_{m}\right\rangle_{m=1}^{\infty}$, such that $\bigcup_{m=1}^{\infty} N_{m}$ is strongly dense in $M$, for each $m \in \mathbb{N}, \quad I \in N_{m}$, and $\lim _{m \rightarrow \infty} \triangle_{\varphi}\left(N_{m}\right) \leq \alpha$. This is clear for if $M_{0}=\{0\}$, then for each $m$ define

$$
\left.N_{m}=0 \oplus \underset{1 \leq j \leq \min \{m, s\}}{ } M_{k_{j}}(\mathbb{C})\right) \oplus \mathbb{C} \cdot\left(\bigoplus_{m<j \leq s} I_{j}\right)
$$

Observe that

$$
\triangle_{\varphi}\left(N_{m}\right)=1-\sum_{j=1}^{\min \{m, s\}} \frac{\alpha_{j}^{2}}{k_{j}^{2}}-\left(\sum_{m<j \leq s} \alpha_{j}\right)^{2} .
$$

$\lim _{m \rightarrow \infty} \triangle_{\varphi}\left(N_{m}\right)=\alpha$ and all the other properties required of the $N_{m}$ are easily checked. If $M_{0} \neq\{0\}$, then there exists a nested sequence of finite-dimensional *-subalgebras of $M_{0},\left\langle A_{m}\right\rangle_{m=1}^{\infty}$ with $I_{0} \in A_{m}$ for each $m$ and $\bigcup_{m=1}^{\infty} A_{m}$ strongly dense in $M_{0}$. For each $m$ define

$$
N_{m}=A_{m} \oplus\left(\bigoplus_{1 \leq j \leq \min \{m, s\}} M_{k_{j}}(\mathbb{C})\right) \oplus \mathbb{C} \cdot\left(\bigoplus_{m<j \leq s} I_{j}\right)
$$


Observe that

$$
\begin{aligned}
\triangle_{\varphi}\left(N_{m}\right) & =1+\left(\triangle_{\alpha_{0} \varphi_{0}}\left(A_{m}\right)-1\right)-\sum_{j=1}^{\min \{m, s\}} \frac{\alpha_{j}^{2}}{k_{j}^{2}}-\left(\sum_{m<j \leq s} \alpha_{j}\right)^{2} \\
& \leq 1-\sum_{j=1}^{\min \{m, s\}_{j}^{2}} \frac{\alpha_{j}^{2}}{k_{j}^{2}}-\left(\sum_{m<j \leq s} \alpha_{j}\right)^{2} .
\end{aligned}
$$

As $m \rightarrow \infty$ the dominating term above converges to $\alpha$; so $\lim _{m \rightarrow \infty} \triangle_{\varphi}\left(N_{m}\right) \leq \alpha$ (existence of the limit is guaranteed by Lemma 3.8 and the fact that $N_{m} \subset N_{m+1}$ ). All the other properties of the $N_{m}$ are easily checked. Notice that in either case, $\lim _{m \rightarrow \infty} \triangle_{\varphi}\left(N_{m}\right) \leq \alpha$ and Lemma 3.8 imply $\triangle_{\varphi}\left(N_{m}\right) \leq \alpha$ for all $m \in \mathbb{N}$.

Take a sequence $\left\langle N_{m}\right\rangle_{m=1}^{\infty}$ as constructed above. Suppose $\min \{1, \beta / 4, C\}>$ $\epsilon>0$. By Kaplansky's Density Theorem there exists an $m_{0} \in \mathbb{N}$ and selfadjoint $x_{1}, \ldots, x_{n} \in N_{m_{0}}$ satisfying $\left|x_{i}-a_{i}\right|_{2}<\epsilon$ and $\left\|x_{i}\right\| \leq R$ for $1 \leq i \leq n$. Denote by $B$ the $*$-algebra generated by $\left\{x_{1}, \ldots, x_{n}, I\right\}$. By Lemma 3.2 and Lemma 3.7, $\chi\left(a_{1}+\epsilon s_{1}, \ldots, a_{n}+\epsilon s_{n}, I+\epsilon s_{n+1}: s_{1}, \ldots, s_{n+1}\right)$ is dominated by

$$
\begin{aligned}
\limsup _{k \rightarrow \infty}\left[k^{-2}\right. & \left.\cdot \log \left(\operatorname{vol}\left(T\left(x_{1}, \ldots, x_{n}, I ; k, 4 \epsilon\right)\right)\right)+\frac{n+1}{2} \cdot \log k\right] \\
\leq & \log \left((4 \epsilon)^{n+1-\triangle_{\varphi}(B)}\right)+\log D \\
\leq & \log \left(\epsilon^{n+1-\triangle_{\varphi}(B)}\right)+\log \left(4^{n+1} D\right)
\end{aligned}
$$

where

$$
D=\pi^{\frac{n+1}{2}}(8(R+1))^{n+1} C^{\triangle_{\varphi}(B)}\left[(2 e)^{\frac{n+1}{2}}\right] .
$$

Set

$$
D_{0}=\pi^{n+1}(8(R+1))^{n+1}(C+1) 6^{n+1} .
$$

Clearly $D_{0}>D . B \subset N_{m_{0}}$ is a unital inclusion and so by Lemma 3.8, $\triangle_{\varphi}(B) \leq$ $\triangle_{\varphi}\left(N_{m_{0}}\right) \leq \alpha$. Hence, $n+1-\triangle_{\varphi}(B) \geq n+1-\alpha$. Since $0<\epsilon<1$,

$$
\log \left(\epsilon^{n+1-\triangle_{\varphi}(B)}\right)+\log \left(4^{n+1} D\right) \leq \log \left(\epsilon^{n+1-\alpha}\right)+\log \left(4^{n+1} D_{0}\right) .
$$

Thus,

$$
\begin{gathered}
\frac{\chi\left(a_{1}+\epsilon s_{1}, \ldots, a_{n}+\epsilon s_{n}, I+\epsilon s_{n+1}: s_{1}, \ldots, s_{n+1}\right)}{|\log \epsilon|} \\
\leq-(n+1)+\alpha+\frac{\log \left(4^{n+1} D_{0}\right)}{|\log \epsilon|} .
\end{gathered}
$$

$D_{0}$ is independent of $\epsilon$ and so by Lemma 3.9,

$$
\begin{aligned}
\delta_{0}\left(a_{1}, \ldots, a_{n}\right)=\delta_{0}\left(a_{1}, \ldots, a_{n}, I\right) \\
\quad \leq(n+1)+\limsup _{\epsilon \rightarrow 0}\left(-(n+1)+\alpha+\frac{\log \left(4^{n+1} D_{0}\right)}{|\log \epsilon|}\right) \\
\quad=\alpha \\
\quad=1-\sum_{i=1}^{s} \frac{\alpha_{i}^{2}}{k_{i}^{2}} .
\end{aligned}
$$




\section{Weak Hyperfinite Monotonicity}

Throughout this section assume $b_{1}, \ldots, b_{p}$ are selfadjoint elements in $M$ and the strongly closed algebra $B$ generated by the $b_{j}$ is hyperfinite. We will prove that if $\left\{b_{1}, \ldots, b_{p}\right\}$ lies in the $*$-algebra generated by $\left\{a_{1}, \ldots, a_{n}\right\}$, then

$$
\delta_{0}\left(b_{1}, \ldots, b_{p}\right) \leq \delta_{0}\left(a_{1}, \ldots, a_{n}\right) .
$$

This "weak hyperfinite monotonic" inequality has significant implications in finding sharp lower bounds for $\delta_{0}\left(a_{1}, \ldots, a_{n}\right)$ when $M$ is diffuse.

The argument is simple, despite the notation that shrouds it. Because $B$ is hyperfinite, matricial microstates of $\left\{b_{1}, \ldots, b_{p}\right\}$ are all approximately unitarily equivalent; the proof is nothing more than a trivial generalization of Lemma 3.1. It follows that $\delta_{0}\left(b_{1}, \ldots, b_{p}\right)$ reflects the metric entropy of the unitary orbit of a single microstate for $\left\{b_{1}, \ldots, b_{p}\right\}$ (provided the microstate approximates well enough). Since the $b_{j}$ are polynomials of the $a_{i}$ (and thus images of the $a_{i}$ under Lipschitz maps), the metric entropy data carries over to the microstates of $\left\{a_{1}, \ldots, a_{n}\right\}$ and yields lower bounds for the metric entropy of the unitary orbit of a microstate for $\left\{a_{1}, \ldots, a_{n}\right\}$. Stuffing this lower bound information into the modified free entropy dimension machine we arrive at the above inequality.

In addition to maintaining the conventions set forth in Section 2, we adopt the following notation in this section:

- For $r>0,\left(M_{k}^{s a}(\mathbb{C})\right)_{r}$ denotes the operator norm ball of $M_{k}^{s a}(\mathbb{C})$ of radius $r$ centered at the origin. For any $d \in \mathbb{N},\left(\left(M_{k}^{s a}(\mathbb{C})\right)_{r}\right)^{d}$ is the Cartesian product of $d$ copies of $\left(M_{k}^{s a}(\mathbb{C})\right)_{r}$.

- For $d \in \mathbb{N}, K \subset\left(M_{k}^{s a}(\mathbb{C})\right)^{d}$, and $u \in U_{k}$ define $u K u^{*}$ to be the set

$$
\left\{\left(u x_{1} u^{*}, \ldots, u x_{d} u^{*}\right):\left(x_{1}, \ldots, x_{d}\right) \in K\right\} .
$$

- For $d \in \mathbb{N}$ and $\left(x_{1}, \ldots, x_{d}\right) \in\left(M_{k}^{s a}(\mathbb{C})\right)^{d}$ define $U\left(x_{1}, \ldots, x_{d}\right)=\left\{\left(u x_{1} u^{*}\right.\right.$, $\left.\left.\ldots, u x_{n} u^{*}\right): u \in U_{k}\right\}$.

- For $\epsilon>0, d \in \mathbb{N}$, and $S \subset\left(M_{k}^{s a}(\mathbb{C})\right)_{d}$ write $P_{\epsilon}(S)$ for the maximum number of points in an $\epsilon$-separated subset of $S$ and $\mathcal{N}_{\epsilon}(S)$ for the $\epsilon$-neighborhood of $S$, both taken with respect to the metric $\rho\left(\left(x_{1}, \ldots, x_{d}\right),\left(y_{1}, \ldots, y_{d}\right)\right)=$ $\max \left\{\left|x_{i}-y_{i}\right|_{2}: 1 \leq i \leq d\right\}$.

First we show that matricial microstates of $\left\{b_{1}, \ldots, b_{p}\right\}$ are approximately unitarily equivalent. We do this with the following two lemmas, the first of which makes no use of hyperfiniteness.

Lemma 4.1. If $z_{1}, \ldots, z_{p} \in B$ are selfadjoint, $\left\|z_{j}\right\| \leq r$ for $1 \leq j \leq p$, and $L, \gamma_{0}>0$, then there exist polynomials $f_{1}, \ldots, f_{p}$ in $p$ noncommuting variables such that for $1 \leq j \leq p$ :

- $\left|f_{j}\left(b_{1}, \ldots, b_{p}\right)-z_{j}\right|_{2}<2 \gamma_{0}$

- $\left\|f_{j}\left(b_{1}, \ldots, b_{p}\right)\right\| \leq r+1$

- for any $k \in \mathbb{N}$ and $\left(x_{1}, \ldots, x_{p}\right) \in\left(\left(M_{k}^{s a}(\mathbb{C})\right)_{L}\right)^{p}, f_{j}\left(x_{1}, \ldots, x_{p}\right) \in$ $\left(M_{k}^{s a}(\mathbb{C})\right)_{r+1}$.

Proof. By Kaplansky's Density Theorem there exist polynomials $g_{1}, \ldots, g_{p}$ in $p$ noncommuting variables such that for $1 \leq j \leq p$ :

- $\left|g_{j}\left(b_{1}, \ldots, b_{p}\right)-z_{j}\right|_{2}<\gamma_{0}$;

- $\left\|g_{j}\left(b_{1}, \ldots, b_{p}\right)\right\| \leq r$

- $g_{j}\left(y_{1}, \ldots, y_{p}\right)$ is selfadjoint for any selfadjoint operators $y_{1}, \ldots, y_{p}$. 
There exists an $L_{1}>L+r$ such that for any $1 \leq j \leq p$ and $k \in \mathbb{N}$, if $\left(x_{1}, \ldots, x_{p}\right) \in$ $\left(\left(M_{k}^{s a}(\mathbb{C})\right)_{L}\right)^{p}$, then $\left\|g_{j}\left(x_{1}, \ldots, x_{p}\right)\right\| \leq L_{1}$. Define $f:\left[-L_{1}, L_{1}\right] \rightarrow \mathbb{R}$ by

$$
f(t)= \begin{cases}t & \text { if }|t| \leq r, \\ r & \text { if } r<t \leq L_{1}, \\ -r & \text { if }-L_{1} \leq t<-r .\end{cases}
$$

For any $1 \leq j \leq p, f\left(g_{j}\left(b_{1}, \ldots, b_{p}\right)\right)=g_{j}\left(b_{1}, \ldots, b_{p}\right)$ and

- $\left|f\left(g_{j}\left(b_{1}, \ldots, b_{p}\right)\right)-z_{j}\right|_{2}<\gamma_{0}$

- $\left\|f\left(g_{j}\left(b_{1}, \ldots, b_{p}\right)\right)\right\| \leq r$;

- for any $k \in \mathbb{N}$ if $\left(x_{1}, \ldots, x_{p}\right) \in\left(\left(M_{k}^{s a}(\mathbb{C})\right)_{L}\right)^{p}$, then $f\left(g_{j}\left(x_{1}, \ldots, x_{p}\right)\right) \in$ $\left(M_{k}^{s a}(\mathbb{C})\right)_{r}$.

Approximating $f$ uniformly on $\left[-L_{1}, L_{1}\right]$ by a polynomial $h$ (to within sufficiently small $\epsilon>0$ ) and setting $f_{j}=h \circ g_{j}$ yields the desired result.

Lemma 4.2. If $\epsilon>0$ and $r \geq \max \left\{\left\|b_{j}\right\|\right\}_{1 \leq j \leq p}$, then there exist $m \in \mathbb{N}$ and $\gamma>0$ such that for each $k \in \mathbb{N}$ and $\left(x_{1}, \ldots, x_{p}\right),\left(y_{1}, \ldots, y_{p}\right) \in \Gamma_{r}\left(b_{1}, \ldots, b_{p} ; m, k, \gamma\right)$ there exists a $u \in U_{k}$ satisfying $\left|u x_{j} u^{*}-y_{j}\right|_{2}<\epsilon$ for $1 \leq j \leq p$.

Proof. By Kaplansky's Density Theorem and the hyperfiniteness of $B$ there exist selfadjoint elements $z_{1}, \ldots, z_{p} \in B$ that generate a finite-dimensional algebra and such that $\left\|z_{j}\right\| \leq r$ and $\left|z_{j}-b_{j}\right|_{2}<\epsilon$ for all $1 \leq j \leq p$. By the remark following Corollary 3.4 there exist $m_{1} \in \mathbb{N}$ and $\gamma_{1}>0$ such that if $r_{0}=\max \left\{\left\|z_{j}\right\|\right\}_{1 \leq j \leq p}$, $k \in \mathbb{N}$, and $\left(x_{1}, \ldots, x_{p}\right),\left(y_{1}, \ldots, y_{p}\right) \in \Gamma_{r_{0}+1}\left(z_{1}, \ldots, z_{p} ; m_{1}, k, \gamma_{1}\right)$, then there exists a $u \in U_{k}$ satisfying $\left|u x_{j} u^{*}-y_{j}\right|_{2}<\epsilon$ for $1 \leq j \leq p$.

By Lemma 4.1 for $\frac{\epsilon}{2}>\gamma_{0}>0$ there exist polynomials $f_{1}, \ldots, f_{p}$ in $p$ noncommuting variables such that for $1 \leq j \leq p$ :

- $\left|f_{j}\left(b_{1}, \ldots, b_{p}\right)-z_{j}\right|_{2}<2 \gamma_{0}<\epsilon$

- $\left\|f_{j}\left(b_{1}, \ldots, b_{p}\right)\right\| \leq r_{0}+1$;

- for any $k \in \mathbb{N}$, if $\left(x_{1}, \ldots, x_{p}\right) \in\left(\left(M_{k}^{s a}(\mathbb{C})\right)_{r}\right)^{p}$, then $f_{j}\left(x_{1}, \ldots, x_{p}\right) \in$ $\left(M_{k}^{s a}(\mathbb{C})\right)_{r_{0}+1}$.

By making $\gamma_{0}$ sufficiently small it follows that for any $1 \leq j \leq m_{1}$ and $1 \leq$ $i_{1}, \ldots, i_{j} \leq p$,

$$
\left|f_{i_{1}}\left(b_{1}, \ldots, b_{p}\right) \cdots f_{i_{j}}\left(b_{1}, \ldots, b_{p}\right)-z_{i_{1}} \cdots z_{i_{j}}\right|_{2}<\gamma_{1} .
$$

Hence by choosing $m \in \mathbb{N}$ large enough, and $\gamma>0$ small enough, if $k \in \mathbb{N}$ and $\left(x_{1}, \ldots, x_{p}\right) \in \Gamma_{r}\left(b_{1}, \ldots, b_{p} ; m, k, \gamma\right)$, then

$$
\left|f_{j}\left(x_{1}, \ldots, x_{p}\right)-x_{j}\right|_{2} \leq\left|f_{j}\left(b_{1}, \ldots, b_{p}\right)-b_{j}\right|_{2}+\epsilon
$$

for $1 \leq j \leq p$ and

$$
\left(f_{1}\left(x_{1}, \ldots, x_{p}\right), \ldots, f_{p}\left(x_{1}, \ldots, x_{p}\right)\right) \in \Gamma_{r_{0}+1}\left(z_{1}, \ldots, z_{p} ; m_{1}, k, \gamma_{1}\right) .
$$

Finally, suppose $k \in \mathbb{N}$ and $\left(x_{1}, \ldots, x_{p}\right),\left(y_{1}, \ldots, y_{p}\right) \in \Gamma_{r}\left(b_{1}, \ldots, b_{p} ; m, k, \gamma\right)$. For any $1 \leq j \leq p$,

$$
\left|f_{j}\left(x_{1}, \ldots, x_{p}\right)-x_{j}\right|_{2} \leq\left|f_{j}\left(b_{1}, \ldots, b_{p}\right)-b_{j}\right|_{2}+\epsilon \leq\left|z_{j}-b_{j}\right|_{2}+2 \epsilon<3 \epsilon .
$$

Similarly for $1 \leq j \leq p, \quad\left|f_{j}\left(y_{1}, \ldots, y_{p}\right)-y_{j}\right|_{2}<3 \epsilon$. By the preceding two paragraphs there exists a $u \in U_{k}$ such that for $1 \leq j \leq p$,

$$
\left|u f_{j}\left(x_{1}, \ldots, x_{p}\right) u^{*}-f_{j}\left(y_{1}, \ldots, y_{p}\right)\right|_{2}<\epsilon .
$$

So for $1 \leq j \leq p, \quad\left|u x_{j} u^{*}-y_{j}\right|_{2}<7 \epsilon$. 
In the next lemma suppose $r>\max \left\{\left\|b_{j}\right\|\right\}_{1 \leq j \leq p}$.

Lemma 4.3. For each $0<\epsilon<1$ there exist corresponding $m_{\epsilon} \in \mathbb{N}$ and $\gamma_{\epsilon}>0$ such that if $L>0$ and $\left\langle\left(x_{1}^{(k)}, \ldots, x_{p}^{(k)}\right)\right\rangle_{k=1}^{\infty}$ is a sequence satisfying $\left(x_{1}^{(k)}, \ldots, x_{p}^{(k)}\right) \in$ $\left(M_{k}^{s a}(\mathbb{C})\right)^{p}$ for all $k$ and $\left(x_{1}^{(k)}, \ldots, x_{p}^{(k)}\right) \in \Gamma_{r}\left(b_{1}, \ldots, b_{p} ; m_{\epsilon}, k, \gamma_{\epsilon}\right)$ for sufficiently large $k$, then

$$
\begin{aligned}
\limsup _{k \rightarrow \infty}\left[k^{-2}\right. & \left.\cdot \log \left(P_{\epsilon L}\left(U\left(x_{1}^{(k)}, \ldots, x_{p}^{(k)}\right)\right)\right)\right] \\
& \geq \chi_{r}\left(b_{1}+\epsilon s_{1}, \ldots, b_{p}+\epsilon s_{p}: s_{1}, \ldots, s_{p}\right)+p|\log \epsilon|-K_{0}
\end{aligned}
$$

where $K_{0}=p \cdot \log ((2+L) \sqrt{2 \pi e})$.

Proof. Suppose $0<\epsilon<1$. By Lemma 4.2 there exist $m_{\epsilon} \in \mathbb{N}$ and $\gamma_{\epsilon}>0$ such that if $k \in \mathbb{N}$,

$$
\left(z_{1}, \ldots, z_{p}\right) \in \Gamma_{r}\left(b_{1}+\epsilon s_{1}, \ldots, b_{p}+\epsilon s_{p}: s_{1}, \ldots, s_{p} ; m_{\epsilon}, k, \gamma_{\epsilon}\right),
$$

and $\left(x_{1}, \ldots, x_{p}\right) \in \Gamma_{r}\left(b_{1}, \ldots, b_{p} ; m_{\epsilon}, k, \gamma_{\epsilon}\right)$, then there exists a $u \in U_{k}$ satisfying $\left|u x_{j} u^{*}-z_{j}\right|_{2}<2 \epsilon$ for $1 \leq j \leq p$.

Assume that $\left\langle\left(x_{1}^{(k)}, \ldots, x_{p}^{(k)}\right)\right\rangle_{k=1}^{\infty}$ satisfies the hypothesis of the lemma with $m_{\epsilon}$ and $\gamma_{\epsilon}$ chosen according to the preceding paragraph. For sufficiently large $k$,

$$
\Gamma_{r}\left(b_{1}+\epsilon s_{1}, \ldots, b_{p}+\epsilon s_{p}: s_{1}, \ldots, s_{p} ; m_{\epsilon}, k, \gamma_{\epsilon}\right) \subset \mathcal{N}_{2 \epsilon}\left(U\left(x_{1}^{(k)}, \ldots, x_{p}^{(k)}\right)\right) .
$$

Find an $\epsilon L$-separated set $W_{k}$ of $U\left(x_{1}^{(k)}, \ldots, x_{p}^{(k)}\right)$ (with respect to the $\rho$ metric) of maximum cardinality $\mathcal{N}_{2 \epsilon}\left(U\left(x_{1}^{(k)}, \ldots, x_{p}^{(k)}\right)\right) \subset \mathcal{N}_{(2+L) \epsilon}\left(W_{k}\right)$. For large enough $k$, $\operatorname{vol}\left(\Gamma_{r}\left(b_{1}+\epsilon s_{1}, \ldots, b_{p}+\epsilon s_{p}: s_{1}, \ldots, s_{p} ; m_{\epsilon}, k, \gamma_{\epsilon}\right)\right) \leq \operatorname{vol}\left(\mathcal{N}_{(2+L) \epsilon}\left(W_{k}\right)\right)$

$$
\leq\left|W_{k}\right| \cdot \frac{\pi^{\frac{p k^{2}}{2}}((2+L) \epsilon \sqrt{k})^{p k^{2}}}{\Gamma\left(\frac{k^{2}}{2}+1\right)^{p}} .
$$

By the preceding inequality, $\chi_{r}\left(b_{1}+\epsilon s_{1}, \ldots, b_{p}+\epsilon s_{p}: s_{1}, \ldots, s_{p} ; m_{\epsilon}, \gamma_{\epsilon}\right)$ is dominated by

$$
\begin{aligned}
& \limsup _{k \rightarrow \infty}\left[k^{-2} \cdot \log \left|W_{k}\right|+p \log ((2+L) \epsilon \sqrt{\pi k})-p k^{-2} \cdot \log \left(\Gamma\left(\frac{k^{2}}{2}+1\right)\right)+\frac{p}{2} \log k\right] \\
& \leq \limsup _{k \rightarrow \infty}\left[k^{-2} \cdot \log \left|W_{k}\right|+p \log ((2+L) \epsilon \sqrt{\pi})-p k^{-2} \log \left(\left(\frac{k^{2}}{2 e}\right)^{\frac{k^{2}}{2}}\right)+p \log k\right] \\
& =\limsup _{k \rightarrow \infty}\left[k^{-2} \cdot \log \left|W_{k}\right|+p \log ((2+L) \epsilon \sqrt{\pi})-\frac{p}{2} \cdot \log \left(\frac{k^{2}}{2 e}\right)+p \log k\right] \\
& =\limsup _{k \rightarrow \infty}\left[k^{-2} \cdot \log \left|W_{k}\right|+p \log ((2+L) \epsilon \sqrt{\pi})+\frac{p}{2} \log (2 e)\right] \\
& =p \log ((2+L) \sqrt{2 \pi e})+\log \epsilon+\limsup _{k \rightarrow \infty}\left(k^{-2} \cdot \log \left|W_{k}\right|\right) \\
& =K_{0}+p \log \epsilon+\limsup _{k \rightarrow \infty}\left[k^{-2} \cdot \log \left(P_{\epsilon L}\left(U\left(x_{1}^{(k)}, \ldots, x_{p}^{(k)}\right)\right)\right)\right] .
\end{aligned}
$$

By the above calculation, $\chi_{r}\left(b_{1}+\epsilon s_{1}, \ldots, b_{p}+\epsilon s_{p}: s_{1}, \ldots, s_{p}\right)+p|\log \epsilon|-K_{0}$ is dominated by

$$
\limsup _{k \rightarrow \infty}\left[k^{-2} \cdot \log \left(P_{\epsilon L}\left(U\left(x_{1}^{(k)}, \ldots, x_{p}^{(k)}\right)\right)\right)\right] .
$$


Write $A$ for the $*$-algebra generated by $\left\{a_{1}, \ldots, a_{n}\right\}$.

Lemma 4.4. If $\left\{b_{1}, \ldots, b_{p}\right\} \subset A$, then there exists an $L>0$ such that for any $0<\epsilon<1, m \in \mathbb{N}$, and $\gamma>0$ there is a sequence $\left\langle\left(x_{1}^{(k)}, \ldots, x_{n}^{(k)}\right)\right\rangle_{k=1}^{\infty}$ satisfying $\left(x_{1}^{(k)}, \ldots, x_{n}^{(k)}\right) \in\left(M_{k}^{s a}(\mathbb{C})\right)^{n}$ for all $k,\left(x_{1}^{(k)}, \ldots, x_{n}^{(k)}\right) \in \Gamma_{R+1}\left(a_{1}, \ldots, a_{n} ; m, k, \gamma\right)$ for sufficiently large $k$, and

$$
\begin{aligned}
\limsup _{k \rightarrow \infty} & {\left[k^{-2} \cdot \log \left(P_{4 \epsilon \sqrt{n}}\left(U\left(x_{1}^{(k)}, \ldots, x_{n}^{(k)}\right)\right)\right)\right] } \\
& \geq \chi_{\lambda}\left(b_{1}+\epsilon s_{1}, \ldots, b_{p}+\epsilon s_{p}: s_{1}, \ldots, s_{p}\right)+p|\log \epsilon|-K_{1}
\end{aligned}
$$

where $K_{1}=p \cdot \log ((2+4 \sqrt{n} L) \sqrt{2 \pi e}) \quad$ and $\quad \lambda=L(R+1)+\max \left\{\left\|b_{j}\right\|\right\}_{1 \leq j \leq p}$.

Proof. There exist polynomials $f_{1}, \ldots, f_{p}$ in $n$ noncommuting variables and with no constant terms such that for $1 \leq j \leq p, f_{j}\left(a_{1}, \ldots, a_{n}\right)=b_{j}$ and such that $f_{j}$ of an $n$-tuple of selfadjoint operators is a selfadjoint element. There exists a constant $L>0$ such that if $k \in \mathbb{N}$ and $\xi_{1}, \ldots, \xi_{n}, \eta_{1}, \ldots, \eta_{n} \in\left(M_{k}^{s a}(\mathbb{C})\right)_{R+1}$, then for all $1 \leq j \leq p$

$$
\left|f_{j}\left(\xi_{1}, \ldots, \xi_{n}\right)-f_{j}\left(\eta_{1}, \ldots, \eta_{n}\right)\right|_{2} \leq L \cdot \max \left\{\left|\xi_{i}-\eta_{i}\right|_{2}: 1 \leq i \leq n\right\}
$$

and $\left\|f_{j}\left(\xi_{1}, \ldots, \xi_{n}\right)\right\| \leq L(R+1)$.

Suppose $0<\epsilon<1, m \in \mathbb{N}$, and $\gamma>0$. By Lemma 4.3 there exist an $m_{\epsilon} \in \mathbb{N}$ and $\gamma_{\epsilon}>0$ such that if $\left\langle\left(x_{1}^{(k)}, \ldots, x_{p}^{(k)}\right)\right\rangle_{k=1}^{\infty}$ is a sequence satisfying $\left(x_{1}^{(k)}, \ldots, x_{p}^{(k)}\right) \in$ $\left(M_{k}^{s a}(\mathbb{C})\right)^{p}$ for all $k$ and $\left(x_{1}^{(k)}, \ldots, x_{p}^{(k)}\right) \in \Gamma_{\lambda}\left(b_{1}, \ldots, b_{p} ; m_{\epsilon}, k, \gamma_{\epsilon}\right)$ for sufficiently large $k$, then

$$
\begin{aligned}
\limsup _{k \rightarrow \infty} & {\left[k^{-2} \cdot \log \left(P_{4 \epsilon \sqrt{n} L}\left(U\left(x_{1}^{(k)}, \ldots, x_{p}^{(k)}\right)\right)\right)\right] } \\
& \geq \chi_{\lambda}\left(b_{1}+\epsilon s_{1}, \ldots, b_{p}+\epsilon s_{p}: s_{1}, \ldots, s_{p}\right)+p|\log \epsilon|-K_{1},
\end{aligned}
$$

where $K_{1}=p \cdot \log ((2+4 \sqrt{n} L) \sqrt{2 \pi e})$. By the assumed existence of finite-dimensional approximants for $\left\{a_{1}, \ldots, a_{n}\right\}$ there exists a $k_{0} \in \mathbb{N}$ such that for each $k \geq k_{0}$ there is an $\left(x_{1}^{(k)}, \ldots, x_{n}^{(k)}\right) \in \Gamma_{R+1}\left(a_{1}, \ldots, a_{n} ; m, k, \gamma\right)$ satisfying

$$
\left(f_{1}\left(x_{1}^{(k)}, \ldots, x_{n}^{(k)}\right), \ldots, f_{p}\left(x_{1}^{(k)}, \ldots, x_{n}^{(k)}\right)\right) \in \Gamma_{\lambda}\left(b_{1}, \ldots, b_{p} ; m_{\epsilon}, k, \gamma_{\epsilon}\right) .
$$

For each $k \geq k_{0}$ and $1 \leq j \leq p$, set $y_{j}^{(k)}=f_{j}\left(x_{1}^{(k)}, \ldots, x_{n}^{(k)}\right)$. It follows that

$$
\begin{aligned}
\limsup _{k \rightarrow \infty} & {\left[k^{-2} \cdot \log \left(P_{4 \epsilon \sqrt{n} L}\left(U\left(y_{1}^{(k)}, \ldots, y_{p}^{(k)}\right)\right)\right)\right] } \\
& \geq \chi_{\lambda}\left(b_{1}+\epsilon s_{1}, \ldots, b_{p}+\epsilon s_{p}: s_{1}, \ldots, s_{p}\right)+p|\log \epsilon|-K_{1} .
\end{aligned}
$$

For $k \geq k_{0}$ and any $u, v \in U_{k}, 1 \leq j \leq p$, observe that

$$
L \cdot \max \left\{\left|u x_{i}^{(k)} u^{*}-v x_{i}^{(k)} v^{*}\right|_{2}: 1 \leq i \leq n\right\}
$$

dominates

$$
\left|q_{j}\left(u x_{1}^{(k)} u^{*}, \ldots, u x_{n}^{(k)} u^{*}\right)-q_{j}\left(v x_{1}^{(k)} v^{*}, \ldots, v x_{n}^{(k)} v^{*}\right)\right|_{2}\left|u y_{j}^{(k)} u^{*}-v y_{j}^{(k)} v^{*}\right|_{2} .
$$

It follows that for any $k \geq k_{0}$,

$$
k^{-2} \cdot \log \left(P_{4 \epsilon \sqrt{n}}\left(U\left(x_{1}^{(k)}, \ldots, x_{n}^{(k)}\right)\right)\right) \geq k^{-2} \cdot \log \left(P_{4 \epsilon \sqrt{n} L}\left(U\left(y_{1}^{(k)}, \ldots, y_{p}^{(k)}\right)\right)\right) .
$$

By the last sentence of the preceding paragraph we are done. 
Theorem 4.5. (Weak Hyperfinite Monotonicity) If $\left\{b_{1}, \ldots, b_{p}\right\} \subset A$, then

$$
\delta_{0}\left(b_{1}, \ldots, b_{p}\right) \leq \delta_{0}\left(a_{1}, \ldots, a_{n}\right) .
$$

Proof. Consider the constants $L$ and $\lambda$ corresponding to $b_{1}, \ldots, b_{p}$ in Lemma 4.4. Suppose $0<\gamma, \epsilon<\frac{1}{2}$ and $m \in \mathbb{N}$. By Lemma 4.4 there exists a sequence $\left\langle\left(x_{1}^{(k)}, \ldots, x_{n}^{(k)}\right)\right\rangle_{k=1}^{\infty}$ satisfying $\left(x_{1}^{(k)}, \ldots, x_{n}^{(k)}\right) \in\left(M_{k}^{s a}(\mathbb{C})\right)^{n}$ for all $k \in \mathbb{N},\left(x_{1}^{(k)}, \ldots\right.$, $\left.x_{n}^{(k)}\right) \in \Gamma_{R+1}\left(a_{1}, \ldots, a_{n} ; m, k, \frac{\gamma}{(8(R+2))^{m}}\right)$ for sufficiently large $k$, and

$$
\begin{aligned}
\limsup _{k \rightarrow \infty} & {\left[k^{-2} \cdot \log \left(P_{4 \epsilon \sqrt{n}}\left(U\left(x_{1}^{(k)}, \ldots, x_{n}^{(k)}\right)\right)\right)\right] } \\
& \geq \chi_{\lambda}\left(b_{1}+\epsilon s_{1}, \ldots, b_{p}+\epsilon s_{p}: s_{1}, \ldots, s_{p}\right)+p|\log \epsilon|-K_{1}
\end{aligned}
$$

where $K_{1}=p \cdot \log ((2+4 \sqrt{n} L) \sqrt{2 \pi e})$.

By Corollary 2.14 of [11] there is an $N \in \mathbb{N}$ such that if $k \geq N$ and $\sigma$ is a Radon probability measure on $\left(\left(M_{k}^{s a}(\mathbb{C})\right)_{R+1}\right)^{2 n}$ invariant under the action

$$
\left(\xi_{1}, \ldots, \xi_{n}, \eta_{1}, \ldots, \eta_{n}\right) \mapsto\left(\xi_{1}, \ldots, \xi_{n}, u \eta_{1} u^{*}, \ldots, u \eta_{n} u^{*}\right)
$$

for $u \in U_{k}$, then $\sigma\left(\omega_{k}\right)>\frac{1}{2}$ where

$$
\begin{aligned}
\omega_{k}=\left\{\left(\xi_{1}, \ldots, \xi_{n}, \eta_{1}, \ldots, \eta_{n}\right)\right. & \in\left(\left(M_{k}^{s a}(\mathbb{C})\right)_{R+1}\right)^{2 n}: \\
& \left.\left\{\xi_{1}, \ldots, \xi_{n}\right\} \text { and }\left\{\eta_{1}, \ldots, \eta_{n}\right\} \text { are }\left(m, \frac{\gamma}{4^{m}}\right) \text {-free }\right\} .
\end{aligned}
$$

For $k \in \mathbb{N}$ write $\nu_{k}$ for the atomic probability measure concentrated at $\left(x_{1}^{(k)}, \ldots\right.$, $\left.x_{n}^{(k)}\right)$ and write $m_{k}$ for the probability measure obtained by restricting vol to $\Gamma_{2 \epsilon}\left(\epsilon s_{1}, \ldots, \epsilon s_{n} ; m, k, \frac{\gamma}{8^{m}}\right)$ and normalizing appropriately. $\nu_{k} \times m_{k}$ is a Radon probability measure on $\left(\left(M_{k}^{s a}(\mathbb{C})\right)_{R+1}\right)^{2 n}$ invariant under the $U_{k}$-action described above. Write $F_{k}$ for the set of all $\left(z_{1}, \ldots, z_{n}\right) \in \Gamma_{2 \epsilon}\left(\epsilon s_{1}, \ldots, \epsilon s_{n} ; m, k, \frac{\gamma}{8^{m}}\right)$ such that $\left\{z_{1}, \ldots, z_{n}\right\}$ and $\left\{x_{1}^{(k)}, \ldots, x_{n}^{(k)}\right\}$ are $\left(m, \frac{\gamma}{4^{m}}\right)$-free.

For $k \geq N, \frac{1}{2}<\left(\nu_{k} \times m_{k}\right)\left(\omega_{k}\right)=m_{k}\left(F_{k}\right)$. Set $E_{k}=\left(x_{1}^{(k)}, \ldots, x_{n}^{(k)}\right)+F_{k}$. For $u \in U_{k}, \operatorname{vol}\left(u E_{k} u^{*}\right)=\operatorname{vol}\left(E_{k}\right)$ and $u E_{k} u^{*}$ is contained in

$$
\Gamma_{R+1+2 \epsilon}\left(a_{1}+\epsilon s_{1}, \ldots, a_{n}+\epsilon s_{n}: \epsilon s_{1}, \ldots, \epsilon s_{n} ; m, k, \gamma\right) .
$$

For each $k \in N$ there exists a subset $\left\langle u_{k, s}\right\rangle_{s \in S_{k}}$ of $U_{k}$ such that

$$
\left|S_{k}\right|=P_{4 \epsilon \sqrt{n}}\left(U\left(x_{1}^{(k)}, \ldots, x_{n}^{(k)}\right)\right)
$$

and for $s, s^{\prime} \in S_{k}$ with $s \neq s^{\prime}$,

$$
\max \left\{\left|u_{k, s} x_{i}^{(k)} u_{k, s}^{*}-u_{k, s^{\prime}} x_{i}^{(k)} u_{k, s^{\prime}}^{*}\right|_{2}: 1 \leq i \leq n\right\}>4 \epsilon \sqrt{n} .
$$

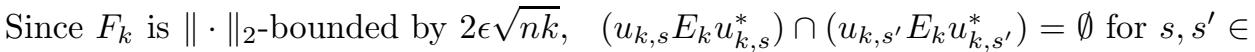
$S_{k}, s \neq s^{\prime}$. Hence for $k \geq N, \operatorname{vol}\left(\Gamma_{R+1+2 \epsilon}\left(a_{1}+\epsilon s_{1}, \ldots, a_{n}+\epsilon s_{n}: \epsilon s_{1}, \ldots, \epsilon s_{n}\right.\right.$; $m, k, \gamma))$ dominates

$$
\begin{aligned}
\operatorname{vol}\left(\bigsqcup_{s \in S_{k}} u_{k, s} E_{k} u_{k, s}^{*}\right) & =\left|S_{k}\right| \cdot \operatorname{vol}\left(F_{k}\right) \\
& =\left|S_{k}\right| \cdot m_{k}\left(F_{k}\right) \cdot \operatorname{vol}\left(\Gamma_{2 \epsilon}\left(\epsilon s_{1}, \ldots, \epsilon s_{n} ; m, k, \gamma / 8^{m}\right)\right) \\
& >1 / 2 \cdot\left|S_{k}\right| \cdot \operatorname{vol}\left(\Gamma_{2 \epsilon}\left(\epsilon s_{1}, \ldots, \epsilon s_{n} ; m, k, \gamma / 8^{m}\right)\right)
\end{aligned}
$$


By the last sentence of the preceding paragraph,

$$
\begin{aligned}
& \chi_{R+1+2 \epsilon}\left(a_{1}+\epsilon s_{1}, \ldots, a_{n}+\epsilon s_{n}: \epsilon s_{1}, \ldots, \epsilon s_{n} ; m, \gamma\right) \\
\geq & \limsup _{k \rightarrow \infty}\left(k^{-2} \cdot\left[\log \left(1 / 2 \cdot\left|S_{k}\right| \cdot \operatorname{vol}\left(\Gamma_{2 \epsilon}\left(\epsilon s_{1}, \ldots, \epsilon s_{n} ; m, k, \gamma / 8^{m}\right)\right)\right)\right]+n / 2 \cdot \log k\right) \\
\geq & \limsup _{k \rightarrow \infty}\left(k^{-2} \cdot \log \left(\left|S_{k}\right|\right)\right) \\
& +\liminf _{k \rightarrow \infty}\left(k^{-2} \cdot\left[\log \left(\operatorname{vol}\left(\Gamma_{2 \epsilon}\left(\epsilon s_{1}, \ldots, \epsilon s_{n} ; m, k, \gamma / 8^{m}\right)\right)\right)\right]+n / 2 \cdot \log k\right) \\
\geq & \limsup _{k \rightarrow \infty}\left(k^{-2} \cdot \log \left(\left|S_{k}\right|\right)\right)+\chi_{2 \epsilon}\left(\epsilon s_{1}, \ldots, \epsilon s_{n}\right) \\
= & \limsup _{k \rightarrow \infty}\left[k^{-2} \cdot \log \left(P_{4 \epsilon \sqrt{n}}\left(U\left(x_{1}^{(k)}, \ldots, x_{n}^{(k)}\right)\right)\right)\right]+n \log (\epsilon \sqrt{2 \pi e}) \\
\geq \quad & \chi\left(b_{1}+\epsilon s_{1}, \ldots, b_{p}+\epsilon s_{p}: s_{1}, \ldots, s_{p}\right)+p|\log \epsilon|+n \log (\epsilon \sqrt{2 \pi e})-K_{1},
\end{aligned}
$$

where we used regularity of $\left\{\epsilon s_{1}, \ldots, \epsilon s_{n}\right\}$ going from the third to the fourth lines above. $m$ and $\gamma$ being arbitrary it follows that

$$
\begin{aligned}
\chi\left(a_{1}+\epsilon s_{1}, \ldots, a_{n}+\epsilon s_{n}: s_{1}, \ldots, s_{n}\right) \geq & \chi\left(b_{1}+\epsilon s_{1}, \ldots, b_{p}+\epsilon s_{p}: s_{1}, \ldots, s_{p}\right) \\
& +(p-n) \cdot|\log \epsilon|+n \cdot \log (\sqrt{2 \pi e})-K_{1} .
\end{aligned}
$$

Dividing by $|\log \epsilon|$, taking limsup's as $\epsilon \rightarrow 0$, and adding $n$ to both sides yields

$$
\delta_{0}\left(a_{1}, \ldots, a_{n}\right) \geq \delta_{0}\left(b_{1}, \ldots, b_{p}\right) .
$$

Corollary 4.6. If $a \in M$, then $\delta_{0}\left(a_{1}, \ldots, a_{n}\right) \geq \delta_{0}(a)$.

Proof. Find a sequence $\left\langle z_{k}\right\rangle_{k=1}^{\infty}$ in $A$ such that $z_{k} \rightarrow a$ strongly. By Proposition 6.14 of [9] and Corollary 6.7 of [10], $\liminf _{k \rightarrow \infty} \delta_{0}\left(z_{k}\right)=\liminf _{k \rightarrow \infty} \delta\left(z_{k}\right) \geq \delta(a)=$ $\delta_{0}(a)$. For each $k, z_{k}$ generates a hyperfinite von Neumann algebra; by Lemma 4.2, for each $k, \delta_{0}\left(a_{1}, \ldots, a_{n}\right) \geq \delta_{0}\left(z_{k}\right)$. So the preceding sentence yields the desired result.

Corollary 4.7. If $M$ has a diffuse von Neumann subalgebra, then $\delta_{0}\left(a_{1}, \ldots, a_{n}\right)$ $\geq 1$.

Proof. Find a maximal abelian subalgebra $N$ of the diffuse von Neumann subalgebra. $N$ has a selfadjoint generator $a$. $N$ must be diffuse since it is a maximal abelian subalgebra of a diffuse von Neumann algebra. Consequently, $a$ has no eigenvalues. Apply Corollary 4.6.

Remark 4.8. By [10] if $M$ has a regular diffuse von Neumann subalgebra, then $\delta_{0}\left(a_{1}, \ldots, a_{n}\right) \leq 1$. By [4] if there exists a sequence of Haar unitaries $\left\langle u_{j}\right\rangle_{j=1}^{s}$ such that the sequence generates $M$ as a von Neumann algebra and for each $j \in \mathbb{N}$, $u_{j+1} u_{j} u_{j+1}^{*} \in\left\{u_{1}, \ldots, u_{j}\right\}^{\prime \prime}$, then $\delta_{0}\left(a_{1}, \ldots, a_{n}\right) \leq 1$. Combining these results with Corollary 4.7, it follows that for any selfadjoint generators $a_{1}, \ldots, a_{n}$ for an $M$ that satisfies either of the two conditions and that is also embeddable into the ultraproduct of the hyperfinite $I I_{1}$-factor, $\delta_{0}\left(a_{1}, \ldots, a_{n}\right)=1$. In other words, $\delta_{0}$ is a von Neumann algebra invariant for such algebras. In particular, $\delta_{0}(M)=1$ when $M$ can be embedded into the ultraproduct of the hyperfinite $I I_{1}$-factor and $M$ has a Cartan subalgebra, $M=N_{1} \otimes N_{2}$ for $I I_{1}$-factors $N_{1}$ and $N_{2}$, or when $M$ is a group von Neumann algebra associated to the groups $S L_{n}(\mathbb{Z}), n \geq 3$. 


\section{Lower Bound for Finite-Dimensional Algebras}

In this section we calculate the lower bound for $\delta_{0}\left(a_{1}, \ldots, a_{n}\right)$ when $M$ is finite dimensional. Without loss of generality, assume throughout this section that $M=$ $\bigoplus_{i=1}^{p} M_{k_{i}}(\mathbb{C})$ and $\varphi=\bigoplus_{i=1}^{p} \alpha_{i} t r_{k_{i}}$ where $p \in \mathbb{N}$ and $\alpha_{i}>0$ for each $i$. The first lemma we present is not necessary but it is convenient.

Lemma 5.1. There exists an $x \in M$ such that the *-algebra generated by $x$ is $M$.

The proof is not hard and we omit it.

As in the preceding section, the calculation of the lower bound amounts to looking at the packing number of unitary orbits of microstates. We use two ingredients.

For a representation $\pi: M \rightarrow M_{k}(\mathbb{C})$ define $H_{\pi}$ to be the unitary group of $(\pi(M))^{\prime}$ and $X_{\pi}=U_{k} / H_{\pi}$. Endow $X_{\pi}$ with the quotient metric from the $|\cdot|_{2^{-}}$ metric on $U_{k}$. Denote this metric on $X_{\pi}$ by $d_{\pi}$. The first ingredient is a packing number estimate for certain homogeneous spaces $X_{\pi}$.

Lemma 5.2. There exists $a \kappa>0$ with the property that for every $\varepsilon>0$ there is a corresponding sequence $\left\langle\sigma_{k}\right\rangle_{k=1}^{\infty}$ such that for each $k, \sigma_{k}: M \rightarrow M_{k}(\mathbb{C})$ is a *-homomorphism and for $k$ sufficiently large:

- $\left\|t r_{k} \circ \sigma_{k}-\varphi\right\|<\varepsilon$.

- For each $k$, setting $H_{k}=H_{\sigma_{k}}$ and $X_{k}=X_{\sigma_{k}}$ we have that $H_{k}$ is a tractable Lie subgroup of $U_{k}$ satisfying $k^{2}\left(\triangle_{\varphi}(M)-\varepsilon\right) \leq \operatorname{dim}\left(X_{k}\right)$.

- For any $\epsilon>0$,

$$
\left(\frac{\kappa}{\epsilon}\right)^{\operatorname{dim} X_{k}} \leq P\left(X_{k}, \epsilon\right)
$$

where $P\left(X_{k}, \epsilon\right)$ is the maximum number of points in an $\epsilon$-separated subset of $X_{k}$.

We quarantine the proof of Lemma 5.2 to the Addendum, merely noting for now that the argument will require some technical modifications to the proofs in 7 .

From now on fix $x$ as in Lemma 5.1. Given a representation $\pi: M \rightarrow M_{k}(\mathbb{C})$, define $U_{\pi}(x)=\left\{u \pi(x) u^{*}: u \in U_{k}\right\}$ and endow $U_{\pi}(x)$ with the inherited $|\cdot|_{2}$-metric. For $u \in U_{k}$ denote $\dot{u}$ to be the image of $u$ in $X_{\pi}$ and define $f_{\pi}: U_{\pi}(x) \rightarrow X_{\pi}$ by $f_{\pi}\left(u \pi(x) u^{*}\right)=\dot{u} . \quad f_{\pi}$ is well defined for if $u, v \in U_{k}, \dot{u}=\dot{v} \Longleftrightarrow v^{*} u \in H_{\pi} \Longleftrightarrow$ $u \pi(x) u *=v \pi(x) v *$.

For the second ingredient recall that in Section 3 covering number estimates with respect to the induced operator norm metrics yield the desired upper bounds for $\delta_{0}\left(a_{1}, \ldots, a_{n}\right)$. Part of the explanation for this lies in the trivial observation that if $u, v \in U_{k}$ and $z \in M_{k}(\mathbb{C})$, then $\left|u z u^{*}-v z v^{*}\right|_{2} \leq 2\|u-v\| \cdot|z|_{2}$. The second ingredient more or less says the reverse: there exists a constant $L>0$ such that $d_{\pi}(\dot{u}, \dot{v}) \leq L \cdot\left|u \pi(x) u^{*}-v \pi(x) v^{*}\right|_{2}$.

Lemma 5.3. If $z, p \in M_{k}(\mathbb{C})$ with $p$ a projection and $z z^{*}, z^{*} z \leq\left\|z^{*} z\right\| p$, then there exists a $y \in M_{k}(\mathbb{C})$ satisfying $y y^{*}=y^{*} y=p$ and

$$
|y-z|_{2} \leq\left|p-z^{*} z\right|_{2}+|p-e|_{2} \leq 2\left|p-z^{*} z\right|_{2}
$$

where $e$ is the projection onto the range of $z^{*} z$.

Proof. Denote the polar decomposition of $z$ by $z=u|z|$ and use the spectral theorem to write $|z|=\sum_{j=1}^{m} \beta_{j} e_{j}$ where the $e_{j}$ are mutually orthogonal rank one 
projections satisfying $e_{1}+\cdots+e_{m}=p$ and $\beta_{j} \geq 0$. Now estimate:

$$
\begin{aligned}
|z-u|_{2}^{2}=|u| z|-u p|_{2}^{2} \leq|| z|-p|_{2}^{2}=\frac{1}{k} \cdot \sum_{j=1}^{m}\left(1-\beta_{j}\right)^{2} & \leq \frac{1}{k} \cdot \sum_{j=1}^{m}\left(1-\beta_{j}\right)^{2}\left(1+\beta_{j}\right)^{2} \\
& =\left|p-z^{*} z\right|_{2}^{2} .
\end{aligned}
$$

$|z-u|_{2} \leq\left|p-z^{*} z\right|_{2}$. Since $u u^{*}, u^{*} u \leq p$ there exists a partial isometry $v$ such that $v v^{*}=p-u u^{*}$ and $v^{*} v=p-u^{*} u$. So if $y=u+v$, then $y y^{*}=y^{*} y=p . u^{*} u=e$ whence

$$
\begin{aligned}
|z-y|_{2} \leq|z-u|_{2}+|v|_{2} & \leq\left|p-z^{*} z\right|_{2}+\left(\operatorname{tr}_{k}\left(v^{*} v\right)\right)^{\frac{1}{2}} \\
& =\left|p-z^{*} z\right|_{2}+\left(\operatorname{tr}_{k}\left(p-u^{*} u\right)\right)^{\frac{1}{2}} \\
& =\left|p-z^{*} z\right|_{2}+|p-e|_{2} \\
& \leq 2\left|p-z^{*} z\right|_{2} .
\end{aligned}
$$

Using Lemma 5.3 we obtain the second ingredient:

Lemma 5.4. $\left\{f_{\pi}:\right.$ for some $k \in \mathbb{N}, \pi: M \rightarrow M_{k}(\mathbb{C})$ is a representation $\}$ is uniformly Lipschitz.

Proof. Suppose $\pi: M \rightarrow M_{k}(\mathbb{C})$ is a representation. Because $|\cdot|_{2}$ is unitarily invariant it suffices to show that for any $u \in U_{k}$,

$$
\inf _{h \in H_{\pi}}|u-h|_{2}=d_{\pi}\left(f_{\pi}\left(u \pi(x) u^{*}\right), f_{\pi}(\pi(x))\right) \leq L \cdot\left|u \pi(x) u^{*}-\pi(x)\right|_{2}
$$

where $L>0$ is a constant dependent only on $x$.

If $u \in U_{k}$, then set $\epsilon=\left|u \pi(x) u^{*}-\pi(x)\right|_{2}$. Denote $\left\langle e_{j l}^{(i)}\right\rangle_{1 \leq i \leq p, 1 \leq j, l \leq k_{i}}$ to be the canonical system of matrix units for $M$. There exist polynomials in two noncommuting variables $\left\langle q_{j l}^{(i)}\right\rangle_{1 \leq i \leq p, 1 \leq j, l \leq k_{i}}$ such that for any $i, j$, and $l, q_{j l}^{(i)}\left(x, x^{*}\right)=e_{j l}^{(i)}$. Set $y_{j l}^{(i)}=\pi\left(e_{j l}^{(i)}\right)$. There exists a constant $C>0$ dependent only on $x$ such that for any $i, j$, and $l$,

$$
\left|u y_{j l}^{(i)}-y_{j l}^{(i)} u\right|_{2}=\left|u y_{j l}^{(i)} u^{*}-y_{j l}^{(i)}\right|_{2} \leq C \epsilon .
$$

Set $K=\sum_{i=1}^{p} k_{i}$. By the above inequality, $\left|u \pi(I) u^{*}-\pi(I)\right|_{2}<C K \epsilon$. Setting $f$ to be the projection onto the orthogonal complement of the range of $\pi(I),\left|u f u^{*}-f\right|_{2}<$ $C K \epsilon$. Now

$$
\begin{aligned}
& \left|u-\left[\left(\sum_{1 \leq i \leq p, 1 \leq j \leq k_{i}} y_{j j}^{(i)} u y_{j j}^{(i)}\right)+f u f\right]\right|_{2} \\
& \quad \leq\left(\sum_{\substack{1 \leq i \leq p, 1 \leq j \leq k_{i}\\
}}\left|u y_{j j}^{(i)}-y_{j j}^{(i)} u\right|_{2} \cdot\left\|y_{j j}^{(i)}\right\|\right)+|u f-f u|_{2} \cdot\|f\| \\
& \quad 2 C K \epsilon .
\end{aligned}
$$

For any $1 \leq i \leq p, 1 \leq j, l \leq k_{i}$,

$$
\begin{aligned}
\left|y_{j j}^{(i)} u y_{j j}^{(i)}-y_{j l}^{(i)} u y_{l j}^{(i)}\right|_{2} & \leq\left|y_{j j}^{(i)} u y_{j j}^{(i)}-y_{j j}^{(i)} u\right|_{2}+\left|y_{j l}^{(i)} y_{l j}^{(i)} u-y_{j l}^{(i)} u y_{l j}^{(i)}\right|_{2} \\
& \leq\left|u y_{j j}^{(i)}-y_{j j}^{(i)} u\right|_{2}+\left|y_{l j}^{(i)} u-u y_{l j}^{(i)}\right|_{2} \\
& \leq 2 C \epsilon .
\end{aligned}
$$


By Lemma 5.3 there exists for each $1 \leq i \leq p$ a $v_{i} \in M_{k}(\mathbb{C})$ such that $v_{i} v_{i}^{*}=v_{i}^{*} v_{i}=$ $y_{11}^{(i)}$ and $\left|v_{i}-y_{11}^{(i)} u y_{11}^{(i)}\right|_{2} \leq 2\left|y_{11}^{(i)} u y_{11}^{(i)} u^{*} y_{11}^{(i)}-y_{11}^{(i)}\right|_{2}$. So

$$
\left|v_{i}-y_{11}^{(i)} u y_{11}^{(i)}\right|_{2} \leq 2\left\|y_{11}^{(i)}\right\|^{2} \cdot\left|u y_{11}^{(i)} u^{*}-y_{11}^{(i)}\right|_{2} \leq 2 C \epsilon .
$$

Similarly there exists a $v \in M_{k}(\mathbb{C})$ such that $v^{*} v=v v^{*}=f$ and $|v-f u f|_{2} \leq$ $2\left|f u f u^{*} f-f\right|_{2} \leq 2 C K \epsilon$.

Consider $z=\left(\sum_{1 \leq i \leq p, 1 \leq j \leq k_{i}} y_{j 1}^{(i)} v_{i} y_{1 j}^{(i)}\right)+v$. It is easy to check that $z$ is a unitary and, because $z$ commutes with all the $y_{j l}^{(i)}, z \in H_{\pi}$. Finally by the last three inequalities of the preceding paragraph,

$$
\begin{aligned}
|u-z|_{2} \leq & \left|u-\left[\left(\sum_{1 \leq i \leq p, 1 \leq j \leq k_{i}} y_{j j}^{(i)} u y_{j j}^{(i)}\right)+f u f\right]\right|_{2} \\
& +\left(\sum_{1 \leq i \leq p, 1 \leq j \leq k_{i}}\left|y_{j j}^{(i)} u y_{j j}^{(i)}-y_{j 1}^{(i)} v_{i} y_{1 j}^{(i)}\right|_{2}\right)+|f u f-v|_{2} \\
\leq & 4 C K \epsilon+\sum_{1 \leq i \leq p, 1 \leq j \leq k_{i}}\left(\left|y_{j j}^{(i)} u y_{j j}^{(i)}-y_{j 1}^{(i)} u y_{1 j}^{(i)}\right|_{2}+\left|y_{j 1}^{(i)} u y_{1 j}^{(i)}-y_{j 1}^{(i)} v_{i} y_{1 j}^{(i)}\right|_{2}\right) \\
\leq & 4 C K \epsilon+\sum_{1 \leq i \leq p, 1 \leq j \leq k_{i}}\left(2 C \epsilon+\left|y_{11}^{(i)} u y_{11}^{(i)}-v_{i}\right|_{2}\right) \\
\leq & 8 C K \epsilon .
\end{aligned}
$$

Set $L=8 C K$, observe that $L$ is dependent only on $x$, and that $\inf _{h \in H_{\pi}}|u-h|_{2} \leq$ $|u-z|_{2} \leq L \epsilon$.

Denote $L>0$ to be the uniform Lipschitz constant of Lemma 5.4. There exists a polynomial $f$ in $n$ noncommuting variables satisfying $f\left(a_{1}, \ldots, a_{n}\right)=x$. There exists an $L_{1}>0$ such that for any $k \in \mathbb{N}$ and $\xi_{1}, \ldots, \xi_{n}, \eta_{1}, \ldots, \eta_{n} \in\left(M_{k}^{s a}(\mathbb{C})\right)_{R}$,

$$
\left|f\left(\xi_{1}, \ldots, \xi_{n}\right)-f\left(\eta_{1}, \ldots, \eta_{n}\right)\right|_{2} \leq L_{1} \cdot \max \left\{\left|\xi_{i}-\eta_{i}\right|_{2}: 1 \leq i \leq n\right\} .
$$

Denote $P_{\epsilon}(S)$ and $U\left(x_{1}, \ldots, x_{d}\right)$ to have the same meaning as in Section 4.

Lemma 5.5. If $\rho_{1}, \rho_{2}>0, m \in \mathbb{N}$, and $\gamma>0$, then there is an $N \in \mathbb{N}$ such that for each $k \geq N$ there exists an $\left(x_{1}^{(k)}, \ldots, x_{n}^{(k)}\right) \in \Gamma_{R}\left(a_{1}, \ldots, a_{n} ; m, k, \gamma\right)$ satisfying for any $\frac{\kappa}{\rho_{1} L L_{1}}>\epsilon>0$,

$$
k^{-2} \cdot \log \left(P_{\epsilon \rho_{1}}\left(U\left(x_{1}^{(k)}, \ldots, x_{n}^{(k)}\right)\right)\right) \geq\left(\triangle_{\varphi}(M)-\rho_{2}\right) \cdot \log \left(\frac{\kappa}{\rho_{1} L L_{1} \epsilon}\right) .
$$

Proof. By Lemma 5.2 there is an $N \in \mathbb{N}$ such that for each $k \geq N$ there exists a *-homomorphism $\sigma_{k}: M \rightarrow M_{k}(\mathbb{C})$ satisfying:

- $\left\|t r_{k} \circ \sigma_{k}-\varphi\right\| \leq \frac{\gamma}{(R+1)^{m}}$.

- For each $k$, setting $H_{k}=H_{\sigma_{k}}$ and $X_{k}=X_{\sigma_{k}}$ we have that $H_{k}$ is a tractable Lie subgroup of $U_{k}$ and $k^{2}\left(\triangle_{\varphi}(M)-\rho_{2}\right) \leq \operatorname{dim}\left(X_{k}\right)$.

- For $\epsilon>0$,

$$
\left(\frac{\kappa}{\epsilon}\right)^{\operatorname{dim} X_{k}} \leq P\left(X_{k}, \epsilon\right)
$$

where $P\left(X_{k}, \epsilon\right)$ is the maximum number of points in an $\epsilon$-separated subset of $X_{k}$. 
For each $1 \leq i \leq n$ and $k \geq N$, define $x_{i}^{(k)}=\sigma_{k}\left(a_{i}\right) \in M_{k}^{s a}(\mathbb{C})$. By the first condition above, $\left(x_{1}^{(k)}, \ldots, x_{n}^{(k)}\right) \in \Gamma_{R}\left(a_{1}, \ldots, a_{n} ; m, k, \gamma\right)$. To see that the second condition is fulfilled suppose $\epsilon$ satisfies the hypothesis of the lemma and $k \geq N$. $\kappa \geq \rho_{1} L L_{1} \epsilon$ and so

$$
P\left(X_{k}, \rho_{1} L L_{1} \epsilon\right) \geq\left(\frac{\kappa}{\rho_{1} L L_{1} \epsilon}\right)^{\operatorname{dim} X_{k}} \geq\left(\frac{\kappa}{\rho_{1} L L_{1} \epsilon}\right)^{k^{2}\left(\triangle_{\rho}(M)-\rho_{2}\right)} .
$$

For any $u, v \in U_{k}$,

$$
\begin{aligned}
d_{\sigma_{k}}(\dot{u}, \dot{v}) & \leq L \cdot\left|u \sigma_{k}(x) u^{*}-v \sigma_{k}(x) v^{*}\right|_{2} \\
& =L \cdot\left|f\left(u \sigma_{k}\left(a_{1}\right) u^{*}, \ldots, u \sigma_{k}\left(a_{n}\right) u^{*}\right)-f\left(v \sigma_{k}\left(a_{1}\right) v^{*}, \ldots, v \sigma_{k}\left(a_{n}\right) v^{*}\right)\right|_{2} \\
& \leq L L_{1} \cdot \max \left\{\left|u x_{i}^{(k)} u^{*}-v x_{i}^{(k)} v^{*}\right|_{2}: 1 \leq i \leq n\right\} .
\end{aligned}
$$

It follows that $P_{\epsilon \rho_{1}}\left(U\left(x_{1}^{(k)}, \ldots, x_{n}^{(k)}\right)\right) \geq P\left(X_{k}, \rho_{1} L L_{1} \epsilon\right)$. Hence for $k \geq N$,

$$
k^{-2} \cdot \log \left(P_{\epsilon \rho_{1}}\left(U\left(x_{1}^{(k)}, \ldots, x_{n}^{(k)}\right)\right)\right) \geq\left(\triangle_{\varphi}(M)-\rho_{2}\right) \cdot \log \left(\frac{\kappa}{\rho_{1} L L_{1} \epsilon}\right) .
$$

The following corollary will not be used until the next section. Suppose $r>R$.

Corollary 5.6. If $\Omega>0$ and $\frac{\kappa}{3 \Omega L L_{1}}>\epsilon>0$, then there exist $m \in \mathbb{N}$ and $\gamma>0$ such that if $\left\langle\left(x_{1}^{(k)}, \ldots, x_{n}^{(k)}\right)\right\rangle_{k=1}^{\infty}$ is a sequence satisfying $\left(x_{1}^{(k)}, \ldots, x_{n}^{(k)}\right) \in\left(M_{k}^{s a}(\mathbb{C})\right)^{n}$ for all $k$, and $\left(x_{1}^{(k)}, \ldots, x_{n}^{(k)}\right) \in \Gamma_{r}\left(a_{1}, \ldots, a_{n} ; m, k, \gamma\right)$ for sufficiently large $k$, then

$$
k^{-2} \cdot \log \left(P_{\Omega \epsilon}\left(U\left(x_{1}^{(k)}, \ldots, x_{n}^{(k)}\right)\right)\right) \geq\left(\triangle_{\varphi}(M)-\epsilon\right) \cdot \log \left(\frac{\kappa}{3 L L_{1} \Omega \epsilon}\right)
$$

for sufficiently large $k$.

Proof. By Corollary 3.4 there exist $m \in \mathbb{N}$ and $\gamma>0$ such that for any $k \in \mathbb{N}$ and $\left(y_{1}, \ldots, y_{n}\right),\left(z_{1}, \ldots, z_{n}\right) \in \Gamma_{r}\left(a_{1}, \ldots, a_{n} ; m, k, \gamma\right)$ there exists a $u \in U_{k}$ such that for $\left.1 \leq j \leq n, \mid u y_{j} u^{*}-z_{j}\right)\left.\right|_{2}<\frac{\epsilon}{2}$. By Lemma 5.5 there exists an $N \in \mathbb{N}$ such that for each $k \geq N$ there exists an $\left(z_{1}^{(k)}, \ldots, z_{n}^{(k)}\right) \in \Gamma_{r}\left(a_{1}, \ldots, a_{n} ; m, k, \gamma\right)$ satisfying

$$
k^{-2} \cdot \log \left(P_{3 \Omega \epsilon}\left(U\left(z_{1}^{(k)}, \ldots, z_{n}^{(k)}\right)\right)\right) \geq\left(\triangle_{\varphi(M)}-\epsilon\right) \cdot\left(\frac{\kappa}{3 L L_{1} \Omega \epsilon}\right) .
$$

Now merely observe that for any such sequence $\left\langle\left(x_{1}^{(k)}, \ldots, x_{n}^{(k)}\right)\right\rangle_{k=1}^{\infty}$ satisfying the hypothesis of the corollary with $m$ and $\gamma$ chosen above, $P_{\Omega \epsilon}\left(U\left(x_{1}^{(k)}, \ldots, x_{n}^{(k)}\right)\right) \geq$ $P_{3 \Omega \epsilon}\left(U\left(z_{1}^{(k)}, \ldots, z_{n}^{(k)}\right)\right)$ for $k \geq N$.

Theorem 5.7. $\delta_{0}\left(a_{1}, \ldots, a_{n}\right) \geq 1-\sum_{i=1}^{p} \frac{\alpha_{i}^{2}}{k_{i}^{2}}$.

Proof. Suppose $\min \left\{\frac{1}{2}, \frac{\kappa}{4 \sqrt{n} L L_{1}}\right\}>\epsilon>0, m \in \mathbb{N}$, and $\gamma, r>0$. Corollary 2.14 of [11] provides an $N \in \mathbb{N}$ such that if $k \geq N$ and $\sigma$ is a Radon probability measure on $\left(\left(M_{k}^{s a}(\mathbb{C})\right)_{R+1}\right)^{2 n}$ invariant under the $U_{k}$-action $\left(\xi_{1}, \ldots, \xi_{n}, \eta_{1}, \ldots, \eta_{n}\right) \mapsto$ $\left(\xi_{1}, \ldots, \xi_{n}, u \eta_{1} u^{*}, \ldots, u \eta_{n} u^{*}\right)$ where $u \in U_{k}$, then $\sigma\left(\omega_{k}\right)>\frac{1}{2}$ where

$$
\begin{aligned}
\omega_{k}=\left\{\left(\xi_{1}, \ldots, \xi_{n}, \eta_{1}, \ldots, \eta_{n}\right)\right. & \in\left(\left(M_{k}^{s a}(\mathbb{C})\right)_{R+1}\right)^{2 n}: \\
& \left.\left\{\xi_{1}, \ldots, \xi_{n}\right\} \text { and }\left\{\eta_{1}, \ldots, \eta_{n}\right\} \text { are }\left(m, \frac{\gamma}{4^{m}}\right) \text {-free }\right\} .
\end{aligned}
$$


Lemma 5.5 provides an $N_{1} \in \mathbb{N}$ such that for each $k \geq N_{1}$ there exists an $\left(x_{1}^{(k)}, \ldots, x_{n}^{(k)}\right) \in \Gamma_{R}\left(a_{1}, \ldots, a_{n} ; m, k, \gamma /(8(R+2))^{m}\right)$ satisfying

$$
k^{-2} \cdot \log \left(P_{4 \epsilon \sqrt{n}}\left(U\left(x_{1}^{(k)}, \ldots, x_{n}^{(k)}\right)\right)\right) \geq\left(\triangle_{\varphi}(M)-r\right) \cdot \log \left(\frac{\kappa}{4 \sqrt{n} L L_{1} \epsilon}\right) .
$$

For $k \geq N+N_{1}$ denote by $\nu_{k}$ the atomic probability measure on $\left(\left(M_{k}^{s a}(\mathbb{C})\right)_{R+1}\right)^{n}$ concentrated at $\left(x_{1}^{(k)}, \ldots, x_{n}^{(k)}\right)$ and denote by $m_{k}$ the Radon probability measure obtained by restricting vol to $\Gamma_{2 \epsilon}\left(\epsilon s_{1}, \ldots, \epsilon s_{n} ; m, k, \gamma / 8^{m}\right)$ and normalizing appropriately. $\nu_{k} \times m_{k}$ is a Radon probability measure on $\left(\left(M_{k}^{s a}(\mathbb{C})\right)_{R+1}\right)^{2 n}$ invariant under the $U_{k}$-action described above. Write $F_{k}$ for the set of all $\left(y_{1}, \ldots, y_{n}\right) \in$ $\Gamma_{2 \epsilon}\left(\epsilon s_{1}, \ldots, \epsilon s_{n} ; m, k, \gamma / 8^{m}\right)$ such that $\left\{y_{1}, \ldots, y_{n}\right\}$ and $\left\{x_{1}^{(k)}, \ldots, x_{n}^{(k)}\right\}$ are $\left(m, \frac{\gamma}{4^{m}}\right)$ free.

For $k \geq N+N_{1}, \quad \frac{1}{2}<\left(\nu_{k} \times m_{k}\right)\left(\omega_{k}\right)=m_{k}\left(F_{k}\right)$. Set $E_{k}=\left(x_{1}^{(k)}, \ldots, x_{n}^{(k)}\right)+$ $F_{k}$. For $k \geq N+N_{1}$ and $u \in U_{k}, \operatorname{vol}\left(u E_{k} u^{*}\right)=\operatorname{vol}\left(F_{k}\right)$ (where $u E_{k} u^{*}$ is defined as in Section 4) and $u E_{k} u^{*}$ is contained in $\Gamma_{R+1}\left(a_{1}+\epsilon s_{1}, \ldots, a_{n}+\epsilon s_{n}\right.$ : $\left.\epsilon s_{1}, \ldots, \epsilon s_{n} ; m, k, \gamma\right)$.

For each $k \geq N+N_{1}$ find a subset $\left\langle u_{k, s}\right\rangle_{s \in S_{k}}$ of $U_{k}$ such that

$$
\left|S_{k}\right|=P_{4 \epsilon \sqrt{n}}\left(U\left(x_{1}^{(k)}, \ldots, x_{n}^{(k)}\right)\right)
$$

and for any $s, s^{\prime} \in S_{k}, s \neq s^{\prime}$,

$$
\max \left\{\left|u_{k, s} x_{i}^{(k)} u_{k, s}^{*}-u_{k, s^{\prime}} x_{i}^{(k)} u_{k, s^{\prime}}^{*}\right|_{2}: 1 \leq i \leq n\right\}>4 \epsilon \sqrt{n} .
$$

$F_{k} \subset\left(M_{k}^{s a}(\mathbb{C})\right)^{n}$ is a $2 \epsilon \sqrt{n k}$ bounded subset with respect to the $\|\cdot\|_{2}$-norm. Hence for any $s, s^{\prime} \in S_{k}, s \neq s^{\prime}\left(u_{k, s} E_{k} u_{k, s}^{*}\right) \cap\left(u_{k, s^{\prime}} E_{k} u_{k, s^{\prime}}^{*}\right)=\emptyset$. Consequently for $k \geq$ $N+N_{1}, \quad \operatorname{vol}\left(\Gamma_{R+1}\left(a_{1}+\epsilon s_{1}, \ldots, a_{n}+\epsilon s_{n}: \epsilon s_{1}, \ldots, \epsilon s_{n} ; m, k, \gamma\right)\right)$ dominates

$$
\begin{aligned}
\operatorname{vol}\left(\bigsqcup_{s \in S_{k}} u_{k, s} E_{k} u_{k, s}^{*}\right)=\left|S_{k}\right| \cdot \operatorname{vol}\left(F_{k}\right) \\
\quad=\left|S_{k}\right| \cdot m_{k}\left(F_{k}\right) \cdot \operatorname{vol}\left(\Gamma_{2 \epsilon}\left(\epsilon s_{1}, \ldots, \epsilon s_{n} ; m, k, \gamma / 8^{m}\right)\right) \\
>\frac{1}{2} \cdot\left|S_{k}\right| \cdot \operatorname{vol}\left(\Gamma_{2 \epsilon}\left(\epsilon s_{1}, \ldots, \epsilon s_{n} ; m, k, \gamma / 8^{m}\right)\right) .
\end{aligned}
$$

By what preceded for $\min \left\{\frac{1}{2}, \frac{\kappa}{4 \sqrt{n} L L_{1}}\right\}>\epsilon>0, m \in \mathbb{N}$, and $\gamma, r>0$, we have that $\chi_{R+1}\left(a_{1}+\epsilon s_{1}, \ldots, a_{n}+\epsilon s_{n}: \epsilon s_{1}, \ldots, \epsilon s_{n} ; m, \gamma\right)$ dominates

$$
\begin{aligned}
& \limsup _{k \rightarrow \infty} k^{-2} \cdot \log \left(\frac{1}{2} \cdot\left|S_{k}\right| \cdot \operatorname{vol}\left(\Gamma_{2 \epsilon}\left(\epsilon s_{1}, \ldots, \epsilon s_{n} ; m, k, \gamma / 8^{m}\right)\right)+\frac{n}{2} \cdot \log k\right) \\
& =\limsup _{k \rightarrow \infty}\left[k^{-2} \cdot \log \left(\operatorname{vol}\left(\Gamma_{2 \epsilon}\left(\epsilon s_{1}, \ldots, \epsilon s_{n} ; m, k, \gamma / 8^{m}\right)\right)+\frac{n}{2} \cdot \log k\right)\right. \\
& \left.+k^{-2} \cdot \log \left(\left|S_{k}\right|\right)\right] \\
& \geq \chi\left(\epsilon s_{1}, \ldots, \epsilon s_{n}\right)+\log \left(\left(\frac{\kappa}{4 \sqrt{n} L L_{1} \epsilon}\right)^{\triangle_{\varphi}(M)-r}\right) \\
& =\log \left(\epsilon^{n+r-\triangle_{\varphi}(M)}\right)+\log \left((2 \pi e)^{\frac{n}{2}}\left(\frac{\kappa}{4 \sqrt{n} L L_{1}}\right)^{\triangle_{\varphi}(M)-r}\right) .
\end{aligned}
$$


Letting $r \rightarrow 0$ it follows that

$$
\begin{aligned}
\chi_{R+1}\left(a_{1}\right. & \left.+\epsilon s_{1}, \ldots, a_{n}+\epsilon s_{n} ; m, \gamma\right) \\
& \geq \log \left(\epsilon^{n-\triangle_{\varphi}(M)}\right)+\log \left((2 \pi e)^{\frac{n}{2}}\left(\frac{\kappa}{4 \sqrt{n} L L_{1}}\right)^{\triangle_{\varphi}(M)}\right) .
\end{aligned}
$$

This inequality holding for all $\epsilon>0$ sufficiently small, $m \in \mathbb{N}$, and $\gamma>0$,

$$
\begin{aligned}
& \chi\left(a_{1}+\epsilon s_{1}, \ldots, a_{n}+\epsilon s_{n}: s_{1}, \ldots, s_{n}\right) \\
& \quad \geq \log \left(\epsilon^{n-\triangle_{\varphi}(M)}\right)+\log \left((2 \pi e)^{\frac{n}{2}}\left(\frac{\kappa}{4 \sqrt{n} L L_{1}}\right)^{\triangle_{\varphi}(M)}\right) .
\end{aligned}
$$

Dividing by $|\log \epsilon|$, taking lim sup's as $\epsilon$ goes to 0 , and adding $n$ to both sides above yields

$$
\delta_{0}\left(a_{1}, \ldots, a_{n}\right) \geq 1-\sum_{i=1}^{p} \frac{\alpha_{i}^{2}}{k_{i}^{2}}
$$

By Theorem 3.10 and Theorem 5.7 we have:

Corollary 5.8. $\delta_{0}\left(a_{1}, \ldots, a_{n}\right)=1-\sum_{i=1}^{p} \frac{\alpha_{i}^{2}}{k_{i}^{2}}$.

\section{The General Lower Bound}

In this section we find a lower bound for $\delta_{0}\left(a_{1}, \ldots, a_{n}\right)$. When $M$ is hyperfinite the lower bound will be sharp. By decomposing $\mathrm{M}$ over its center it follows that

$$
M \simeq M_{0} \oplus\left(\bigoplus_{i=1}^{s} M_{k_{i}}(\mathbb{C})\right) \oplus M_{\infty}, \quad \varphi \simeq \alpha_{0} \varphi_{0} \oplus\left(\bigoplus_{i=1}^{s} \alpha_{i} t r_{k_{i}}\right) \oplus 0
$$

where as in the introduction $s \in \mathbb{N} \cup\{0\} \cup\{\infty\}, \alpha_{i}>0$ for $1 \leq i \leq s(i \in \mathbb{N}), M_{0}$ is a diffuse von Neumann algebra or $\{0\}, \varphi_{0}$ is a faithful, tracial state on $M_{0}$ and $\alpha_{0}>0$ if $M_{0} \neq\{0\}, \varphi_{0}=0$ and $\alpha_{0}=0$ if $M_{0}=\{0\}$, and $M_{\infty}$ is a von Neumann algebra or $\{0\}$. We remark that $M$, hyperfinite or otherwise, always admits such a decomposition. We will show that $\delta_{0}\left(a_{1}, \ldots, a_{n}\right) \geq 1-\sum_{i=1}^{s} \frac{\alpha_{i}^{2}}{k_{i}^{2}}$. Again, because $\varphi$ vanishes on $M_{\infty}$ and our main claim concerns the calculation of a lower bound for $\delta_{0}\left(a_{1}, \ldots, a_{n}\right)$ assume, without loss of generality, that

$$
M=M_{0} \oplus\left(\bigoplus_{i=1}^{s} M_{k_{i}}(\mathbb{C})\right), \quad \varphi=\alpha_{0} \varphi_{0} \oplus\left(\bigoplus_{i=1}^{s} \alpha_{i} t r_{k_{i}}\right) .
$$

We proceed first by finding a suitable set of elements $\left\{a_{1}^{\prime}, a_{2}^{\prime}, a_{3}^{\prime}\right\}$ in the *-algebra generated by the $a_{i}$ such that the packing number of unitary orbits of certain microstates of $\Gamma_{C}\left(a_{1}^{\prime}, a_{2}^{\prime}, a_{3}^{\prime} ; m, k, \gamma\right)$ approximate (from below and in a normalized sense) $1-\sum_{i=1}^{s} \frac{\alpha_{i}^{2}}{k_{i}^{2}}$. These microstates can be obtained as noncommuting polynomials of well-approximating microstates for $\left\{a_{1}, \ldots, a_{n}\right\}$ and hence the packing number of unitary orbits of such microstates of $\Gamma_{C}\left(a_{1}^{\prime}, a_{2}^{\prime}, a_{3}^{\prime} ; m, k, \gamma\right)$ will provide a lower bound for the packing number of unitary orbits of the microstates for $\left\{a_{1}, \ldots, a_{n}\right\}$. One can then use asymptotic freeness results to transform these metric entropy quantities into free entropy dimension quantities as in Theorem 4.5 and Theorem 5.7. 
Throughout this section write $A$ for the $*$-algebra generated by $\left\{a_{1}, \ldots, a_{n}\right\}$. We also maintain the notation introduced at the beginning of Section 4 . In subsections 6.1 and 6.2 we assume that $M_{0} \neq\{0\}$ (which implies $0<\alpha_{0}$ ) and $\alpha_{0}<1$.

6.1. Construction of $\left\{a_{1}^{\prime}, a_{2}^{\prime}, a_{3}^{\prime}\right\}$ when $M_{0} \neq\{0\}$ and $\alpha_{0}<1$. Fix $l \in \mathbb{N}$ with $l \leq s$ and $\varepsilon>0$. Define $M_{1}=\bigoplus_{j=1}^{l} M_{k_{j}}(\mathbb{C})$ and $M_{2}=\bigoplus_{l<j \leq s} M_{k_{j}}(\mathbb{C})$. $M_{1}$ is a finite-dimensional $\mathrm{C}^{*}$-algebra and by Lemma 5.1 has two selfadjoint generators $b_{1}$ and $b_{2} . A$ is strongly dense in $M$; so $A e$ is strongly dense in $M e$ where $e=$ $0 \oplus\left(\bigoplus_{j=1}^{l} I_{j}\right) \oplus 0 \in Z(M)$ and the $I_{j}$ are as in Section 3. Thus $A e=M e$. Consequently there exist $a_{1}^{\prime}, a_{2}^{\prime} \in A$ such that

$$
a_{i}^{\prime}=f_{i} \oplus b_{i} \oplus \xi_{i} \in M_{0} \oplus M_{1} \oplus M_{2}=M .
$$

$M_{0}$ being diffuse, there exists an $f \in M_{0}$ such that $\delta_{0}(f)=1$ and $s p(f)=[1,2]$ (here $\delta_{0}(f)$ is calculated with respect to $\left.\varphi_{0}\right)$. A $A e_{0}$ is strongly dense in $M e_{0}$ where $e_{0}=I_{0} \oplus 0 \in M_{0} \oplus\left(\bigoplus_{1 \leq j \leq s} M_{k_{j}}(\mathbb{C})\right.$. By Kaplansky's Density Theorem, Proposition 6.14 of [8], and Corollary 6.7 of [9] there exists an $0 \leq a \in A$ satisfying:

- $a=g \oplus b \oplus \xi \in M_{0} \oplus M_{1} \oplus M_{2}=M$.

- $s p(g) \subset[0,2], \delta_{0}(g)>1-\varepsilon$, and $\varphi_{0}\left(\chi_{[0,1 / 2]}\right)<\varepsilon$ where for any Borel subset $S \subset \mathbb{R}, \chi_{S}$ denotes the spectral projection of $g$ associated to the set $S$.

- $\|b\|<\frac{1}{6}$.

Since $b \geq 0$ and $M_{1}$ is finite dimensional, $s p(b)=\left\{\beta_{1}, \ldots, \beta_{d}\right\} \subset \mathbb{R}$ where $0 \leq \beta_{1}<\ldots<\beta_{d} \leq \frac{1}{6}$. Define $h:[0,2] \rightarrow \mathbb{R}$ by $h(t)=\left(t-\beta_{1}\right) \cdots\left(t-\beta_{d}\right)$. Observe that:

- $h(b)=0$

- $h^{-1}\left(h\left(\left[0,2 \beta_{d}\right]\right)\right) \subset\left[0,3 \beta_{d}\right] \subset[0,1 / 2]$;

- $h^{-1}\left(h\left(\left(2 \beta_{d}, 2\right]\right)\right) \subset\left(\beta_{d}, 2\right]$.

The third observation implies that if $\beta \in h\left(2 \beta_{d}, 2\right]$, then $h^{-1}(\beta)$ consists of exactly one point in $\left(\beta_{d}, 2\right]$ since $h$ is strictly increasing on $\left(\beta_{d}, 2\right]$. Noting that for all but countably many $\beta, \quad \chi_{h^{-1}\{\beta\}}=0$,

$$
\begin{aligned}
\sum_{\beta \in s p(h(b))} \varphi_{0}\left(\chi_{h^{-1}\{\beta\}}\right)^{2} & =\sum_{\beta \in h(s p(b))} \varphi_{0}\left(\chi_{h^{-1}\{\beta\}}\right)^{2} \\
& \leq \sum_{\beta \in h\left(\left[0,2 \beta_{d}\right]\right)} \varphi_{0}\left(\chi_{h^{-1}\{\beta\}}\right)^{2}+\sum_{\beta \in h\left(\left(2 \beta_{d}, 2\right]\right)} \varphi_{0}\left(\chi_{h^{-1}\{\beta\}}\right)^{2} \\
& \leq \varphi_{0}\left(\sum_{\beta \in h\left(\left[0,2 \beta_{d}\right]\right)} \chi_{h^{-1}\{\beta\}}\right)+\sum_{\beta \in[0,2]} \varphi_{0}\left(\chi_{\{\beta\}}\right)^{2} \\
& \leq \varphi_{0}\left(\chi_{\left[0,3 \beta_{d}\right]}\right)+\varepsilon \\
& <2 \varepsilon .
\end{aligned}
$$

Define $a_{3}^{\prime}=h(a)=h(g) \oplus 0 \oplus h(\xi)$. We have just proven:

Lemma 6.1. If $l \in \mathbb{N}, 1 \leq l \leq s$, and $\varepsilon>0$, then there exist $a_{1}^{\prime}, a_{2}^{\prime}, a_{3}^{\prime} \in A$ of the form $a_{i}^{\prime}=f_{i} \oplus b_{i} \oplus \xi_{i} \in M_{0} \oplus M_{1} \oplus M_{2}=M$ satisfying:

- $\left\{b_{1}, b_{2}\right\}$ generates $M_{1}$ and $b_{3}=0$;

- $\delta_{0}\left(f_{3}\right)>1-\varepsilon$. 
6.2. Lower Bounds Estimates for $\delta_{0}\left(a_{1}, \ldots, a_{n}\right)$ when $M_{0} \neq\{0\}$ and $\alpha_{0}<1$. Fix $l \in \mathbb{N}, l \leq s$, and $\varepsilon>0$. Find elements $a_{1}^{\prime}, a_{2}^{\prime}, a_{3}^{\prime} \in A$ with the properties listed in Lemma 6.1 and denote $M_{1}, M_{2}$ to have the same meaning as in the preceding subsection. Suppose $C \geq \max \left\{\left\|a_{i}^{\prime}\right\|\right\}_{i=1,2,3}+1$. Denote by $\varphi_{1}$ the tracial state on $M_{1}$ obtained by restricting $\varphi$ to $0 \oplus M_{1} \oplus 0$ and normalizing appropriately. Similarly denote by $\varphi_{2}$ the positive trace (possibly 0) on $M_{2}$ obtained by restricting $\varphi$ to $0 \oplus 0 \oplus M_{2}$ and normalizing appropriately. Define $\beta_{0}=\alpha_{0}, \beta_{1}=\sum_{i=1}^{l} \alpha_{i}$, $\beta=\min \left\{\beta_{0}, \beta_{1}\right\}$, and $\beta_{2}=1-\beta_{0}-\beta_{1}$. Recall the constant $\kappa, L$, and $L_{1}$ of the previous section with respect to $M_{1}, \varphi_{1}$, and $x=b_{1}+i b_{2}$. Finally define $e_{0}$ and $e_{1}$ to be the projections $I_{0} \oplus 0 \oplus 0,0 \oplus\left(\oplus_{j=1}^{l} I_{j}\right) \oplus 0 \in M$, respectively, and $e_{2}=I-e_{0}-e_{1}$.

Lemma 6.2. If $D>0$ and $\min \left\{\frac{\beta \kappa}{27 D L L_{1}}, 1\right\}>\epsilon>0$, then there exist $m_{\epsilon} \in \mathbb{N}$ and $\gamma_{\epsilon}>0$ (dependent on $\epsilon$ ) such if $\left\langle\left(z_{1}^{(k)}, z_{2}^{(k)}, z_{3}^{(k)}\right)\right\rangle_{k=1}^{\infty}$ is a sequence satisfying $\left(z_{1}^{(k)}, z_{2}^{(k)}, z_{3}^{(k)}\right) \in\left(M_{k}^{s a}(\mathbb{C})\right)^{3}$ for all $k$ and $\left(z_{1}^{(k)}, z_{2}^{(k)}, z_{3}^{(k)}\right) \in \Gamma_{C}\left(a_{1}^{\prime}, a_{2}^{\prime}, a_{3}^{\prime}:\right.$ $\left.e_{0}, e_{1}, e_{2} ; m_{\epsilon}, k, \gamma_{\epsilon}\right)$ for sufficiently large $k$, then

$$
\limsup _{k \rightarrow \infty} k^{-2} \cdot \log \left(P_{D \epsilon}\left(U\left(z_{1}^{(k)}, z_{2}^{(k)}, z_{3}^{(k)}\right)\right)\right)
$$

dominates

$$
\begin{aligned}
\left(\beta_{0}+\beta_{1}-\epsilon\right)^{2} & \cdot\left(\chi\left(\left(f_{3} \oplus 0\right)+\epsilon s: s\right)+|\log \epsilon|-K\right) \\
+ & \left(\beta_{1}-\epsilon\right)^{2}\left(\triangle_{\varphi_{1}}\left(M_{1}\right)-\epsilon\right) \cdot \log \left(\frac{\beta \kappa}{27 \epsilon D L L_{1}}\right)
\end{aligned}
$$

where $K=\log \left(\left(2+9 \beta^{-1} D\right) \sqrt{2 \pi e}\right)$ and $s$ is a semicircular element free with respect to $M_{0} \oplus M_{1}$.

Proof. Suppose $\min \left\{\frac{\beta \kappa}{27 D L L_{1}}, 1\right\}>\epsilon>0$. By Lemma 4.3 there exist an $m_{1} \in \mathbb{N}$ and $\gamma_{1}$ (dependent on $\epsilon$ ) such that if $\left\langle x^{(k)}\right\rangle_{k=1}^{\infty}$ is a sequence satisfying $x^{(k)} \in M_{k}^{s a}(\mathbb{C})$ for all $k \in \mathbb{N}$ and $x^{(k)} \in \Gamma_{C}\left(f_{3} \oplus 0 ; m_{1}, k, \gamma_{1}\right)$ for sufficiently large $k$, then

$$
\limsup _{k \rightarrow \infty}\left[k^{-2} \cdot \log P_{9 \beta^{-1} D \epsilon}\left(U\left(x^{(k)}\right)\right)\right] \geq \chi_{C}\left(\left(f_{3} \oplus 0\right)+\epsilon s: s\right)+|\log \epsilon|-K
$$

where $K=\log \left(\left(2+9 \beta^{-1} D\right) \sqrt{2 \pi e}\right), f_{3} \oplus 0 \in M_{0} \oplus M_{1}, M_{0} \oplus M_{1}$ is endowed with the tracial state $\left(\beta_{0}+\beta_{1}\right)^{-1}\left(\beta_{0} \varphi_{0} \oplus \beta_{1} \varphi_{1}\right)$, and $s$ is a semicircular element free with respect to $M_{0} \oplus M_{1}$.

By Corollary 5.6 there exist an $m_{2} \in \mathbb{N}$ and $\gamma_{2}>0$ such that if $\left\langle\left(y_{1}^{(k)}, y_{2}^{(k)}\right)\right\rangle_{k=1}^{\infty}$ is a sequence satisfying $\left(y_{1}^{(k)}, y_{2}^{(k)}\right) \in\left(M_{k}^{s a}(\mathbb{C})\right)^{2}$ for all $k$ and $\left(y_{1}^{(k)}, y_{2}^{(k)}\right) \in \Gamma_{C}\left(b_{1}, b_{2}\right.$; $m_{2}, k, \gamma_{2}$ ) for sufficiently large $k$, then

$$
k^{-2} \cdot \log P_{9 \beta^{-1} D \epsilon}\left(U\left(y_{1}^{(k)}, y_{2}^{(k)}\right)\right) \geq \log \left(\left(\frac{\beta \kappa}{27 \epsilon D L L_{1}}\right)^{\triangle_{\varphi_{1}}\left(M_{1}\right)-\epsilon}\right)
$$

for sufficiently large $k$.

By standard approximations for any $\varepsilon>0$ there exist $m \in \mathbb{N}$ and $\gamma>0$ such that for any $k \in \mathbb{N}$ if $\left(z_{1}, z_{2}, z_{3}\right) \in \Gamma_{C}\left(a_{1}^{\prime}, a_{2}^{\prime}, a_{3}^{\prime}: e_{0}, e_{1}, e_{2} ; m, k, \gamma\right)$, then the following conditions are satisfied: 
- There exist mutually orthogonal projections $q_{0}, q_{1}, q_{2} \in M_{k}(\mathbb{C})$ with $n_{0}+$ $n_{1}+n_{2}=k$ where $n_{i}$ denotes the dimension of the range of $q_{i}$ and for each $i,\left|\operatorname{tr}_{k}\left(q_{i}\right)-\beta_{i}\right|<\varepsilon$.

- Canonically identifying $q_{0} M_{k}(\mathbb{C}) q_{0}+q_{1} M_{k}(\mathbb{C}) q_{1}+q_{2} M_{k}(\mathbb{C}) q_{2}$ with $\bigoplus_{i=0}^{2} M_{n_{i}}(\mathbb{C})\left|z_{i}-h_{i}\right|_{2}<\varepsilon$ where $h_{i}=x_{i} \oplus y_{i} \oplus \eta_{i} \in \bigoplus_{i=0}^{2} M_{n_{i}}(\mathbb{C}) \subset M_{k}(\mathbb{C})$ and $y_{3}=0$.

- $x_{3} \oplus 0 \in \Gamma_{C}\left(f_{3} \oplus 0 ; m_{1}, n_{0}+n_{1}, \gamma_{1}\right)$.

- $\left(y_{1}, y_{2}\right) \in \Gamma_{C}\left(b_{1}, b_{2} ; m_{2}, n_{1}, \gamma_{2}\right)$ where $b_{1}, b_{2} \in\left(M, \varphi_{1}\right)$.

Secondly, given $\varepsilon>0$ there exists an $N \in \mathbb{N}$ such that for each $k>N$ there exists a corresponding $k<\tau(k) \in \mathbb{N}$ satisfying $\left|\frac{k}{\tau(k)}-\left(\beta_{0}+\beta_{1}\right)\right|<\varepsilon$. Combining these two remarks, there exist $m_{\epsilon} \in \mathbb{N}$ and $\gamma_{\epsilon}>0$ such that for any $k \in \mathbb{N}$ sufficiently large there exists a $k<\tau(k) \in \mathbb{N}$ such that if $\left(z_{1}, z_{2}, z_{3}\right) \in \Gamma_{C}\left(a_{1}^{\prime}, a_{2}^{\prime}, a_{3}^{\prime}\right.$ : $\left.e_{0}, e_{1}, e_{2} ; m_{\epsilon}, \tau(k), \gamma_{\epsilon}\right)$, then the following conditions are satisfied:

- There exist mutually orthogonal projections $q_{0}, q_{1}, q_{2} \in M_{\tau(k)}(\mathbb{C})$ with $n_{0}+$ $n_{1}+n_{2}=\tau(k)$ where $n_{i}$ denotes the dimension of the range of $q_{i}$ and for each $i,\left|t r_{\tau(k)}\left(q_{i}\right)-\beta_{i}\right|<\frac{1}{2} \cdot \min \{\epsilon, \beta\}$.

- $n_{0}+n_{1}=k$.

- Canonically identifying $q_{0} M_{\tau(k)}(\mathbb{C}) q_{0}+q_{1} M_{\tau(k)}(\mathbb{C}) q_{1}+q_{2} M_{\tau(k)}(\mathbb{C}) q_{2}$ with $\bigoplus_{i=0}^{2} M_{n_{i}}(\mathbb{C}),\left|z_{i}-h_{i}\right|_{2}<\frac{\epsilon}{2}$ where $h_{i}=x_{i} \oplus y_{i} \oplus \eta_{i} \in \bigoplus_{i=0}^{2} M_{n_{i}}(\mathbb{C}) \subset$ $M_{\tau(k)}(\mathbb{C})$ and $y_{3}=0$.

- $x_{3} \oplus 0 \in \Gamma_{C}\left(f_{3} \oplus 0 ; m_{1}, k, \gamma_{1}\right)$.

- $\left(y_{1}, y_{2}\right) \in \Gamma_{C}\left(b_{1}, b_{2} ; m_{2}, n_{1}, \gamma_{2}\right)$ where $b_{1}, b_{2} \in\left(M, \varphi_{1}\right)$.

Now fix $k$ and $\left(z_{1}, z_{2}, z_{3}\right)$ satisfying the aforementioned conditions so that the five properties listed just above hold. Let $n_{i}, h_{i}, x_{i}, y_{i}$, and $\eta_{i}$ correspond to the fixed $k$ and $\left(z_{1}, z_{2}, z_{3}\right)$. Find a set of unitaries $\left\langle u_{s}\right\rangle_{s \in S_{k}}$ of $U_{k}$ such that $|S|=$ $P_{9 \beta^{-1} D \epsilon}\left(U\left(x_{3} \oplus 0\right)\right)$ and for any $s, s^{\prime} \in S$ and $s \neq s^{\prime}$,

$$
\left|u_{s}\left(x_{3} \oplus 0\right) u_{s}^{*}-u_{s^{\prime}}\left(x_{3} \oplus 0\right) u_{s^{\prime}}^{*}\right|_{2}>9 \beta^{-1} D \epsilon .
$$

Find a set of unitaries $\left\langle v_{g}\right\rangle_{g \in G}$ of $U_{n_{1}}$ such that $|G|=P_{9 \beta^{-1} D \epsilon}\left(U\left(y_{1}, y_{2}\right)\right)$ and for any $g, g^{\prime} \in G$ and $g \neq g^{\prime}$,

$$
\max \left\{\left|v_{g} y_{i} v_{g}^{*}-v_{g^{\prime}} y_{i} v_{g,}^{*}\right|_{2}: i=1,2\right\}>9 \beta^{-1} D \epsilon .
$$

For $(s, g) \in S_{k} \times G_{k}$ define $w_{s, g} \in U_{\tau(k)}$ by $w_{s, g}=\left(u_{s}\left(I_{n_{0}} \oplus v_{g}\right)\right) \oplus I_{n_{2}} \in U_{\tau(k)}$.

I claim that the family $\left\langle\left(w_{s, g} h_{1} w_{s, g}^{*}, w_{s, g} h_{2} w_{s, g}^{*}, w_{s, g} h_{3} w_{s, g}^{*}\right)\right\rangle_{s, g} \in S \times G$ is a $3 D \epsilon$-separated set (with respect to the $\rho$ metric defined in section 4). Suppose $(s, g),\left(s^{\prime}, g^{\prime}\right) \in S \times G$ and $(s, g) \neq\left(s^{\prime}, g^{\prime}\right)$. Then either $s \neq s^{\prime}$ or $g \neq g^{\prime}$. In the former case,

$$
\begin{aligned}
\mid w_{s, g} & h_{3} w_{s, g}^{*}-\left.w_{s^{\prime}, g^{\prime}} h_{3} w_{s^{\prime}, g^{\prime}}^{*}\right|_{2} \\
& =\left|w_{s, g}\left(x_{3} \oplus 0 \oplus \eta_{3}\right) w_{s, g}^{*}-w_{s^{\prime}, g^{\prime}}\left(x_{3} \oplus 0 \oplus \eta_{3}\right) w_{s^{\prime}, g^{\prime}}^{*}\right|_{2} \\
& =\left|u_{s}\left(x_{3} \oplus 0\right) u_{s}^{*} \oplus 0-u_{s^{\prime}}\left(x_{3} \oplus 0\right) u_{s^{\prime}}^{*} \oplus 0\right|_{2} \\
& >\sqrt{\frac{\beta_{1}+\beta_{2}}{2}} \cdot 9 \beta^{-1} D \epsilon \\
& >3 D \epsilon .
\end{aligned}
$$


Suppose $g \neq g^{\prime}$. We can assume $s=s^{\prime}$. For $i=1,2$,

$$
\begin{aligned}
& \left|w_{s, g} h_{i} w_{s, g}^{*}-w_{s, g^{\prime}} h_{i} w_{s, g^{\prime}}^{*}\right|_{2} \\
& \quad=\left|w_{s, g}\left(x_{i} \oplus y_{i} \oplus \eta_{i}\right) w_{s, g}^{*}-w_{s, g^{\prime}}\left(x_{i} \oplus y_{i} \oplus \eta_{i}\right) w_{s, g^{\prime}}^{*}\right|_{2} \\
& \quad=\left|u_{s}\left(x_{i} \oplus v_{g} y_{i} v_{g}^{*}\right) u_{s}^{*} \oplus \eta_{3}-u_{s}\left(x_{i} \oplus v_{g^{\prime}} y_{i} v_{g^{\prime}}^{*}\right) u_{s}^{*} \oplus \eta_{3}\right|_{2} \\
& \quad \geq \sqrt{\frac{\beta_{1}}{2}} \cdot\left|v_{g} y_{i} v_{g}^{*}-v_{g^{\prime}} y_{i} v_{g^{\prime}}^{*}\right|_{2}
\end{aligned}
$$

so that

$$
\begin{aligned}
\max & \left\{\left|w_{s, g} h_{i} w_{s, g}^{*}-w_{s, g^{\prime}} h_{i} w_{s, g^{\prime}}^{*}\right|_{2}: i=1,2\right\} \\
& \geq \sqrt{\frac{\beta_{1}}{2}} \cdot \max \left\{\left|v_{g} y_{i} v_{g}^{*}-v_{g^{\prime}} y_{i} v_{g^{\prime}}^{*}\right|_{2}: i=1,2\right\} \\
& >\sqrt{\frac{\beta_{1}}{2}} \cdot 9 \beta^{-1} D \epsilon \\
& >3 D \epsilon .
\end{aligned}
$$

By the inequalities above, $P_{D \epsilon}\left(U\left(z_{1}, z_{2}, z_{3}\right)\right) \geq P_{3 D \epsilon}\left(U\left(h_{1}, h_{2}, h_{3}\right)\right) \geq|S \times G|$.

Now suppose $\left\langle\left(z_{1}^{(k)}, z_{2}^{(k)}, z_{3}^{(k)}\right)\right\rangle_{k=1}^{\infty}$ is a sequence satisfying the hypothesis of the lemma with $m_{\epsilon}$ and $\gamma_{\epsilon}$ as chosen on the previous page. For $k$ sufficiently large,

$$
\left(z_{1}^{(\tau(k))}, z_{2}^{(\tau(k))}, z_{3}^{(\tau(k))}\right) \in \Gamma_{C}\left(a_{1}^{\prime}, a_{2}^{\prime}, a_{3}^{\prime}: e_{0}, e_{1}, e_{2} ; m_{\epsilon}, \tau(k), \gamma_{\epsilon}\right) .
$$

Thus $\left(z_{1}^{(\tau(k))}, z_{2}^{(\tau(k))}, z_{3}^{(\tau(k))}\right)$ satisfies the five conditions previously stated. For each $k$ sufficiently large consider the corresponding $\tau(k) \in \mathbb{N}$ and denote $n_{i}(k), x^{\left(n_{i}(k)\right)}$, $y_{i}^{\left(n_{i}(k)\right)}, S_{k}$, and $G_{k}$ to be the $n_{i}, x_{i}, y_{i}, S$, and $G$, respectively, associated to $\left(z_{1}^{(\tau(k))}, z_{2}^{(\tau(k))}, z_{3}^{(\tau(k))}\right)$. Then

$$
\begin{aligned}
& \limsup _{k \rightarrow \infty} \tau(k)^{-2} \cdot \log \left(P_{D \epsilon}\left(U\left(z_{1}^{(\tau(k))}, z_{2}^{(\tau(k))}, z_{3}^{(\tau(k))}\right)\right)\right) \\
& \quad \geq \limsup _{k \rightarrow \infty} \tau(k)^{-2} \cdot\left(\log \left|S_{k}\right|+\log \left|G_{k}\right|\right) \\
& \quad \geq \limsup _{k \rightarrow \infty}\left(\tau(k)^{-2} \cdot \log \left|S_{k}\right|\right)+\liminf _{k \rightarrow \infty}\left(\tau(k)^{-2} \cdot \log \left|G_{k}\right|\right) .
\end{aligned}
$$

Set $\mathcal{L}=9 \beta^{-1} D \epsilon$. Then $\lim \sup _{k \rightarrow \infty}\left(\tau(k)^{-2} \cdot \log \left|S_{k}\right|\right)$ dominates

$$
\begin{aligned}
& \left(\beta_{0}+\beta_{1}-\epsilon\right)^{2} \cdot \limsup _{k \rightarrow \infty} \frac{1}{\left(n_{0}(k)+n_{1}(k)\right)^{2}} \cdot \log \left(P_{\mathcal{L}}\left(U\left(x_{3}^{\left(n_{0}(k)\right)} \oplus 0\right)\right)\right) \\
& =\left(\beta_{0}+\beta_{1}-\epsilon\right)^{2} \cdot \limsup _{k \rightarrow \infty} \frac{1}{k^{2}} \cdot \log \left(P_{\mathcal{L}}\left(U\left(x_{3}^{\left(n_{0}(k)\right)} \oplus 0\right)\right)\right) \\
& \geq\left(\beta_{0}+\beta_{1}-\epsilon\right)^{2} \cdot \chi_{C}\left(\left(f_{3} \oplus 0\right)+\epsilon s: s\right)+|\log \epsilon|-K
\end{aligned}
$$

and since $n_{1}(k) \rightarrow \infty$ as $k \rightarrow \infty$,

$$
\begin{aligned}
\liminf _{k \rightarrow \infty}\left(\tau(k)^{-2} \cdot \log \left|G_{k}\right|\right) & \\
& \geq\left(\beta_{1}-\epsilon\right)^{2} \cdot \liminf _{k \rightarrow \infty}\left[n_{1}(k)^{-2} \cdot \log \left(P_{\mathcal{L}}\left(U\left(y_{1}^{\left(n_{1}(k)\right)}, y_{2}^{\left(n_{1}(k)\right)}\right)\right)\right)\right] \\
& \geq\left(\beta_{1}-\epsilon\right)^{2} \cdot \log \left(\left(\frac{\beta \kappa}{27 \epsilon D L L_{1}}\right)^{\triangle_{\varphi_{1}}\left(M_{1}\right)-\epsilon}\right) .
\end{aligned}
$$

The desired result follows. 
We now recycle a familiar argument. There exist polynomials $p_{1}, p_{2}, p_{3}$ in $n$ noncommuting variables such that for $1 \leq j \leq 3, p_{j}\left(a_{1}, \ldots, a_{n}\right)=a_{j}^{\prime}$. Without loss of generality, we can assume the $p_{j}$ take $n$-tuples of selfadjoint elements to selfadjoint elements. Find a constant $1<\mathcal{C}$ such that for any $\left(x_{1}, \ldots, x_{n}\right) \in$ $\left(\left(M_{k}^{s a}(\mathbb{C})\right)_{R+1}\right)^{n},\left\|p_{j}\left(x_{1}, \ldots, x_{n}\right)\right\|<\mathcal{C}$. Also, there exists a $D_{0}>0$ such that if $k \in \mathbb{N}$ and $x_{1}, \ldots, x_{n}, y_{1}, \ldots, y_{n} \in\left(M_{k}^{s a}(\mathbb{C})\right)_{R+1}$, then for all $j$,

$$
\left|p_{j}\left(x_{1}, \ldots, x_{n}\right)-p_{j}\left(y_{1}, \ldots, y_{n}\right)\right|_{2} \leq D_{0} \cdot \max \left\{\left|x_{i}-y_{i}\right|_{2}: 1 \leq i \leq n\right\} .
$$

Lemma 6.3. For $m \in \mathbb{N}, \gamma>0$, and small enough $\epsilon>0$ there is a sequence $\left\langle\left(x_{1}^{(k)}, \ldots, x_{n}^{(k)}\right)\right\rangle_{k=1}^{\infty}$ such that $\left(x_{1}^{(k)}, \ldots, x_{n}^{(k)}\right) \in\left(M_{k}^{\text {sa }}(\mathbb{C})\right)^{n}$ for all $k,\left(x_{1}^{(k)}, \ldots, x_{n}^{(k)}\right)$ $\in \Gamma_{R+1}\left(a_{1}, \ldots, a_{n} ; m, k, \gamma\right)$ for sufficiently large $k$, and

$$
\limsup _{k \rightarrow \infty} k^{-2} \cdot \log \left(P_{4 \epsilon \sqrt{n}}\left(U\left(x_{1}^{(k)}, \ldots, x_{n}^{(k)}\right)\right)\right)
$$

dominates

$$
\begin{aligned}
& \left(\beta_{0}+\beta_{1}-\epsilon\right)^{2} \cdot\left(\chi\left(\left(f_{3} \oplus 0\right)+\epsilon s: s\right)+|\log \epsilon|-K\right) \\
& \quad+\left(\beta_{1}-\epsilon\right)^{2}\left(\triangle_{\varphi_{1}}\left(M_{1}\right)-\epsilon\right) \cdot \log \left(\frac{\beta \kappa}{108 \sqrt{n} \epsilon D_{0} L L_{1}}\right)
\end{aligned}
$$

where $K=\log \left(\left(2+36 \beta^{-1} \sqrt{n} D_{0}\right) \sqrt{2 \pi e}\right)$.

Proof. Suppose $\min \left\{\frac{\beta \kappa}{32 D_{0} \sqrt{n} L L_{1}}, 1\right\}>\epsilon>1$. By Lemma 6.2 there exist $m_{\epsilon} \in \mathbb{N}$ and $\gamma_{\epsilon}>0$ such that if $\left\langle\left(z_{1}^{(k)}, z_{2}^{(k)}, z_{3}^{(k)}\right)\right\rangle_{k=1}^{\infty}$ is a sequence satisfying $\left(z_{1}^{(k)}, z_{2}^{(k)}, z_{3}^{(k)}\right)$ $\in\left(M_{k}^{s a}(\mathbb{C})\right)^{3}$ for all $k$ and $\left(z_{1}^{(k)}, z_{2}^{(k)}, z_{3}^{(k)}\right) \in \Gamma_{C}\left(a_{1}^{\prime}, a_{2}^{\prime}, a_{3}^{\prime}: e_{1}, e_{2}, e_{3} ; m_{\epsilon}, k, \gamma_{\epsilon}\right)$ for sufficiently large $k$, then

$$
\limsup _{k \rightarrow \infty} k^{-2} \cdot \log \left(P_{4 \epsilon \sqrt{n} D_{0}}\left(U\left(z_{1}^{(k)}, z_{2}^{(k)}, z_{3}^{(k)}\right)\right)\right)
$$

dominates

$$
\begin{aligned}
& \left(\beta_{0}+\beta_{1}-\epsilon\right)^{2} \cdot\left(\chi\left(\left(f_{3} \oplus 0\right)+\epsilon s: s\right)+|\log \epsilon|-K\right) \\
& \quad+\left(\beta_{1}-\epsilon\right)^{2}\left(\triangle_{\varphi_{1}}\left(M_{1}\right)-\epsilon\right) \cdot \log \left(\frac{\beta \kappa}{108 \sqrt{n} \epsilon D_{0} L L_{1}}\right) .
\end{aligned}
$$

Because $\left\{a_{1}, \ldots, a_{n}\right\}$ has finite-dimensional approximants, there exists an $N \in \mathbb{N}$ such that for each $k \geq N$ there is an $\left(x_{1}^{(k)}, \ldots, x_{n}^{(k)}\right) \in\left(\left(M_{k}^{s a}(\mathbb{C})\right)_{R+1}\right)^{n}$ satisfying

$$
\left(x_{1}^{(k)}, \ldots, x_{n}^{(k)}\right) \in \Gamma_{R+1}\left(a_{1}, \ldots, a_{n} ; m, k, \gamma\right)
$$

and

$$
\left(y_{1}^{(k)}, y_{2}^{(k)}, y_{3}^{(k)}\right) \in \Gamma_{\mathcal{C}}\left(a_{1}^{\prime}, a_{2}^{\prime}, a_{3}^{\prime}: e_{1}, e_{2}, e_{3} ; m_{\epsilon}, k, \gamma_{\epsilon}\right)
$$

where for any $1 \leq j \leq 3, p_{j}\left(x_{1}^{(k)}, \ldots, x_{n}^{(k)}\right)=y_{j}^{(k)}$. Note that we can use the cutoff constant $\mathcal{C}$ by the argument of Lemma 4.1.

If $u, v \in U_{k}$, then $D_{0} \cdot \max \left\{\left|u x_{i}^{(k)} u^{*}-v x_{i}^{(k)} v^{*}\right|_{2}: 1 \leq i \leq n\right\}$ is greater than

$$
\left|p_{j}\left(u x_{1}^{(k)}, \ldots, u x_{n}^{(k)} u^{*}\right)-p_{j}\left(v x_{1}^{(k)} v^{*}, \ldots, v x_{n}^{(k)} v^{*}\right)\right|_{2}=\left|u y_{j}^{(k)} u^{*}-v y_{j}^{(k)} v^{*}\right|_{2} .
$$

Hence,

$$
\begin{aligned}
\limsup _{k \rightarrow \infty} k^{-2} & \cdot \log \left(P_{4 \epsilon \sqrt{n}}\left(U\left(x_{1}^{(k)}, \ldots, x_{n}^{(k)}\right)\right)\right) \\
& \geq \limsup _{k \rightarrow \infty} k^{-2} \cdot \log \left(P_{4 \epsilon \sqrt{n} D_{0}}\left(U\left(y_{1}^{(k)}, y_{2}^{(k)}, y_{3}^{(k)}\right)\right)\right) .
\end{aligned}
$$


In turn the dominated term is greater than or equal to

$$
\begin{aligned}
& \left(\beta_{0}+\beta_{1}-\epsilon\right)^{2} \cdot\left(\chi\left(\left(f_{3} \oplus 0\right)+\epsilon s: s\right)+|\log \epsilon|-K\right) \\
& +\left(\beta_{1}-\epsilon\right)^{2}\left(\triangle_{\varphi_{1}}\left(M_{1}\right)-\epsilon\right) \cdot \log \left(\frac{\beta \kappa}{108 \sqrt{n} \epsilon D_{0} L L_{1}}\right) .
\end{aligned}
$$

Lemma 6.3 more or less gives the lower bound. We simply use the same sort of arguments which allowed us passage from Lemma 4.4 to Theorem 4.5 and from Lemma 5.5 to Theorem 5.7. Namely Voiculescu's approximate freeness results produce an $\epsilon$-ball, most of whose elements are semicircular microstates trying very hard to be free with respect to a matricial microstate for $\left\{a_{1}, \ldots, a_{n}\right\}$. Adding the $\epsilon$-ball to the single microstate creates microstates for $\left\{a_{1}+\epsilon s_{1}, \ldots, a_{n}+\epsilon s_{n}\right\}$. The $\epsilon$ packing number of the unitary orbit of the microstate yields the same number of disjoint, conjugate balls which are microstates of $\left\{a_{1}+\epsilon s_{1}, \ldots, a_{n}+\epsilon s_{n}\right\}$. Applying $\log$ to this packing number, multiplying by $k^{-2}$ and taking a $\lim \sup$ as $k \rightarrow \infty$, dividing by $|\log \epsilon|$ and taking a limsup as $\epsilon \rightarrow 0$ yields a lower bound for the modified free entropy dimension of the $n$-tuple $\left\{a_{1}, \ldots, a_{n}\right\}$.

By the discussion above $\delta_{0}\left(a_{1}, \ldots, a_{n}\right)$ should dominate the number obtained by taking the majorized quantity of Lemma 6.3 , dividing by $|\log \epsilon|$, and taking a $\lim \sup$ as $\epsilon \rightarrow 0$. The resultant quantity of these successive operations is:

$$
\begin{aligned}
& \left(\beta_{0}+\beta_{1}\right)^{2}\left(\delta_{0}\left(f_{3} \oplus 0\right)\right)+\beta_{1}^{2} \cdot \triangle_{\varphi_{1}}\left(M_{1}\right) \\
& \quad>\left(\beta_{0}+\beta_{1}\right)^{2} \cdot\left[1-\left(\beta_{0}+\beta_{1}\right)^{-2}\left(\varepsilon \beta_{0}^{2}+\beta_{1}^{2}\right)\right]+\beta_{1}^{2} \cdot \triangle_{\varphi_{1}}\left(M_{1}\right) \\
& \quad>\left(\beta_{0}+\beta_{1}\right)^{2}-\left(\varepsilon+\beta_{1}^{2}\right)+\beta_{1}^{2} \cdot\left(1-\sum_{i=1}^{l} \frac{\beta_{1}^{-2} \alpha_{i}^{2}}{k_{i}^{2}}\right) \\
& \quad=\left(\beta_{0}+\beta_{1}\right)^{2}-\varepsilon-\sum_{i=1}^{l} \frac{\alpha_{i}^{2}}{k_{i}^{2}} .
\end{aligned}
$$

Omitting the details of a familiar analysis we conclude:

Theorem 6.4. If $M_{0} \neq\{0\}$ and $\alpha_{0}<1$, then $\delta_{0}\left(a_{1}, \ldots, a_{n}\right) \geq\left(\beta_{0}+\beta_{1}\right)^{2}-\varepsilon-$ $\sum_{i=1}^{l} \frac{\alpha_{i}^{2}}{k_{i}^{2}}$.

Letting $l \rightarrow s$ and $\varepsilon \rightarrow 0$ we arrive at:

Corollary 6.5. If $M_{0} \neq\{0\}$ and $\alpha_{0}<1$, then $\delta_{0}\left(a_{1}, \ldots, a_{n}\right) \geq 1-\sum_{i=1}^{s} \frac{\alpha_{i}^{2}}{k_{i}^{2}}$.

6.3. General Lower Bound Estimates for $\delta_{0}\left(a_{1}, \ldots, a_{n}\right)$. Now we deal with the situation where $M_{0}=\{0\}$ or $\alpha_{0}=1$. Suppose first that $M_{0}=\{0\}$. The lower estimates come even easier for, in this case, $M$ is merely a direct sum of matrix algebras. As in section 6.1 given $l \in \mathbb{N}$ with $l \leq s$ and using the same notation, we can construct two selfadjoint elements $a_{i}^{\prime}=b_{i} \oplus \xi_{i} \in M_{1} \oplus M_{2}$ for $i=1,2$ such that they lie in the $*$-algebra generated by $\left\{a_{1}, \ldots, a_{n}\right\}$ and $b_{1}, b_{2}$ generate $M_{1}$. We have a similar packing number estimate in this case:

Lemma 6.6. For small enough $\epsilon, \gamma>0$ and any $m \in \mathbb{N}$ there is a sequence $\left\langle\left(z_{1}^{\left(\triangle_{k}\right)}, z_{2}^{\left(\triangle_{k}\right)}\right)\right\rangle_{k=1}^{\infty}$ such that $k<\triangle_{k} \in \mathbb{N}$ for all $k,\left(z_{1}^{\left(\triangle_{k}\right)}, z_{2}^{\left(\triangle_{k}\right)}\right) \in M_{\triangle_{k}}^{\text {sa }}(\mathbb{C})$ for all $k$,

$$
\left(z_{1}^{\left(\triangle_{k}\right)}, z_{2}^{\left(\triangle_{k}\right)}\right) \in \Gamma_{C}\left(a_{1}^{\prime}, a_{2}^{\prime} ; m, \triangle_{k}, \gamma\right)
$$


for sufficiently large $k$, and

$$
\triangle_{k}^{-2} \cdot \log \left(P_{16 \epsilon}\left(U\left(z_{1}^{\left(\triangle_{k}\right)}, z_{2}^{\left(\triangle_{k}\right)}\right)\right)\right) \geq\left(\beta_{1}-\epsilon\right)^{2}\left(\triangle_{\varphi_{1}}\left(M_{1}\right)-\epsilon\right) \cdot \log \left(\frac{\kappa}{16 \beta^{-1} \epsilon L L_{1}}\right)
$$

for sufficiently large $k$.

The proof mimics that of Lemma 6.2 but it is even easier. Lemma 6.6 requires that we find good enough microstates for $a_{1}^{\prime}, a_{2}^{\prime}$ and this is easy to do (in light of Lemma 3.6). One does not need to deal with the set-up concerning the $f_{i}$ (they are all 0 ) and the estimates are similar to those of Lemma 6.2. We omit a rigorous proof of Lemma 6.6 here and leave it to the reader. We now run an argument similar to that which followed Lemma 6.2. Dividing the dominated term above by $|\log \epsilon|$ in the conclusion of the statement of Lemma 6.4 and taking a lim sup as $\epsilon$ goes to 0 yields $\beta_{1}^{2} \cdot \triangle_{\varphi_{1}}\left(M_{1}\right)$. Using the same asymptotic freeness results in the paragraphs preceding Theorem 6.4 , it follows that $\delta_{0}\left(a_{1}^{\prime}, a_{2}^{\prime}\right)$ is greater than or equal to this limiting process, i.e., greater than or equal to $\beta_{1}^{2} \cdot \triangle_{\varphi_{1}}\left(M_{1}\right)$. Since $M$ is hyperfinite ( $M$ is a direct summand of matrix algebras), by Theorem 4.5, $\delta_{0}\left(a_{1}, \ldots, a_{n}\right) \geq \delta_{0}\left(a_{1}^{\prime}, a_{2}^{\prime}\right) \geq b_{1}^{2}-\varepsilon-\sum_{i=1}^{l} \frac{\alpha_{i}^{2}}{k_{i}^{2}}$. Then $l$ and $\varepsilon$ being arbitrary, $\delta_{0}\left(a_{1}, \ldots, a_{n}\right) \geq 1-\sum_{i=1}^{s} \frac{\alpha_{i}^{2}}{k_{i}^{2}}$.

Secondly, suppose $\alpha_{0}=1$. By Section $4, \delta_{0}\left(a_{1}, \ldots, a_{n}\right)=1$, which yields the desired lower bound.

Having considered all the cases above we have for any $M$ and $a_{1}, \ldots, a_{n}$ as in Section 2:

Theorem 6.7. $\delta_{0}\left(a_{1}, \ldots, a_{n}\right) \geq 1-\sum_{i=1}^{s} \frac{\alpha_{i}^{2}}{k_{i}^{2}}$.

By Theorem 3.9 we also have:

Corollary 6.8. If $M$ is hyperfinite, then $\delta_{0}\left(a_{1}, \ldots, a_{n}\right)=1-\sum_{i=1}^{s} \frac{\alpha_{i}^{2}}{k_{i}^{2}}$.

In light of Corollary 6.5, if $M$ is hyperfinite, then we define $\delta_{0}(M)=$ $\delta_{0}\left(a_{1}, \ldots, a_{n}\right)$. As in the introduction we remark that every such hyperfinite $M$ has a finite set of selfadjoint generators.

\section{Trivialities and a Final Remark}

In concluding the discussion we make a few simple observations about the preceding results. The first is a strengthening of Theorem 4.5. We start with a generalization of Lemma 3.8.

Corollary 7.1. If $N \subset M$ is a unital inclusion of hyperfinite von Neumann algebras, then $\delta_{0}(N) \leq \delta_{0}(M)$.

Proof. Find selfadjoint generators $a_{1}, \ldots, a_{n}$ for $M$ and $b_{1}, \ldots, b_{p}$ for $N$. By Theorem 4.5 and Corollary 6.8,

$$
\delta_{0}(N)=\delta_{0}\left(b_{1}, \ldots, b_{p}\right) \leq \delta_{0}\left(a_{1}, \ldots, a_{n}, b_{1}, \ldots, b_{p}\right)=\delta_{0}(M) .
$$

We now have:

Corollary 7.2. (Hyperfinite Monotonicity). If $N \subset M$ is a unital inclusion of von Neumann algebras and $N$ is hyperfinite, then $\delta_{0}(N) \leq \delta_{0}\left(a_{1}, \ldots, a_{n}\right)$. 
Proof. We may assume $M=M_{0} \oplus\left(\bigoplus_{i=1}^{s} M_{k_{i}}(\mathbb{C})\right)$ and $\varphi=\alpha_{0} \varphi_{0} \oplus\left(\bigoplus_{i=1}^{s} \alpha_{i} t r_{k_{i}}\right)$ where all quantities are as in Section 6. Define $A=\mathbb{C} I_{0} \oplus\left(\bigoplus_{i=1}^{s} M_{k_{i}}(\mathbb{C})\right) \subset M$. It is easy to see that the von Neumann algebra $\mathcal{R}$ generated by $A \cup N$ is hyperfinite. By decomposing $\mathcal{R}$ over its center and observing that the atomic projections of $Z(\mathcal{R})$ contain those of $Z(M)$, it follows from Theorem 6.7 and Corollary 6.8 that $\delta_{0}(\mathcal{R}) \leq$ $\delta_{0}\left(a_{1}, \ldots, a_{n}\right)$. Hence by Corollary $7.1, \delta_{0}(N) \leq \delta_{0}(\mathcal{R}) \leq \delta_{0}\left(a_{1}, \ldots, a_{n}\right)$.

Our second observation is a weak lower semicontinuity property for $\delta_{0}$.

Lemma 7.3. If $\left\langle\left(b_{1}^{(k)}, \ldots, b_{n}^{(k)}\right)\right\rangle_{k=1}^{\infty}$ is a sequence of $n$-tuples of selfadjoint elements in $M$ such that for each $1 \leq i \leq n, b_{i}^{(k)} \rightarrow b_{i}$ strongly and $\left\{b_{1}, \ldots, b_{n}\right\}$ generates a diffuse von Neumann algebra, then

$$
\liminf _{k \rightarrow \infty} \delta_{0}\left(b_{1}^{(k)}, \ldots, b_{n}^{(k)}\right) \geq 1 .
$$

In particular, if $1=\delta_{0}\left(b_{1}, \ldots, b_{n}\right)$, then

$$
\liminf _{k \rightarrow \infty} \delta_{0}\left(b_{1}^{(k)}, \ldots, b_{n}^{(k)}\right) \geq \delta_{0}\left(b_{1}, \ldots, b_{n}\right) .
$$

Proof. Suppose $\varepsilon>0$. By the proof of Corollary 4.7, it follows that there exists a $b=b^{*} \in W^{*}\left(b_{1}, \ldots, b_{n}\right)$ such that $\delta_{0}(b)=1$. There exists a sequence of noncommuting polynomials in $n$ variables $\left\langle q_{m}\right\rangle_{m=1}^{\infty}$ such that $q_{m}\left(b_{1}, \ldots, b_{n}\right)^{*}=$ $q_{m}\left(b_{1}, \ldots, b_{n}\right) \rightarrow b$ strongly. It follows from Proposition 6.14 of [9] and Corollary 6.7 of 10] that for $m$ sufficiently large, $\delta_{0}\left(q_{m}\left(b_{1}, \ldots, b_{n}\right)\right)>1-\frac{\varepsilon}{2}$. Pick one such $m$ and call it $m_{0}$. The same proposition of [9] and corollary of [10] provide a corresponding $N$ such that for all $k>N, \delta_{0}\left(q_{m_{0}}\left(b_{1}^{(k)}, \ldots, b_{n}^{(k)}\right)\right)>1-\varepsilon$. By Corollary 4.6 for all $k>N, \delta_{0}\left(b_{1}^{(k)}, \ldots, b_{n}^{(k)}\right) \geq \delta_{0}\left(q_{m_{0}}\left(b_{1}^{(k)}, \ldots, b_{n}^{(k)}\right)\right)>1-\varepsilon$.

Finally, we comment on the work carried out by Ken Dykema concerning free products of hyperfinite von Neumann algebras with tracial, faithful states. In [2] Dykema investigated the free product of two such algebras $A$ and $B$. There it was shown that $A * B$ was isomorphic to $L\left(F_{s}\right) \oplus C$ where $L\left(F_{s}\right)$ is an interpolated free group factor and $C$ is a finite-dimensional von Neumann algebra. Moreover, Dykema provided formulas for determining $C$ in terms of the matricial parts of $A$ and $B$ and calculating $s$ in terms of the "free dimensions" of $A, B$, and $C$. Given a hyperfinite $M$ as above, Dykema defined the free dimension of $M, \operatorname{fdim}(M)$ to be

$$
\alpha_{0}^{2}+\sum_{i=1}^{s} \alpha_{i}^{2}\left(1-k_{i}^{-2}\right)+2 \alpha_{0}\left(1-\alpha_{0}\right)+\sum_{1 \leq i, j \leq s, i \neq j} \alpha_{i} \alpha_{j} .
$$

Using the identity $1=\left(\sum_{i=0}^{s} \alpha_{i}\right)^{2}$ one finds that the number above equals $\delta_{0}(M)$. In other words, for a hyperfinite von Neumann algebra $M$ with a tracial, faithful state, the quantity $\delta_{0}(M)$ equals the quantity $\operatorname{fdim}(M)$.

\section{Addendum}

In this final section we prove the metric entropy estimates of Lemmas 3.5 and 5.2. The proofs are essentially those of Szarek ([7]) with the addition of the explicit computations of Raymond ([5]).

Throughout $H$ will denote a closed Lie subgroup of $U_{k}$. Define $X=U_{k} / H,|\cdot|_{\infty}$ to be the operator norm, $\mathcal{H}$ to be the Lie subalgebra of $H$ identified in $i M_{k}^{s a}(\mathbb{C})=\mathcal{G}$, and $\mathcal{X}$ to be the orthogonal complement of $\mathcal{H}$ with respect to the real inner product 
on $\mathcal{G}$ generated by $\operatorname{Re} \operatorname{Tr}$. Denote $d_{\infty}$ and $d_{2}$ to be the metrics on $X$ induced by $|\cdot|_{\infty}$ and $|\cdot|_{2}$, respectively. Lastly, for a metric $d$ on a space $\Omega$ and $\epsilon>0$ define $N(\Omega, d, \epsilon)$ to be the minimum number of open $\epsilon$-balls required to cover $\Omega$ with respect to $d$ and $P(\Omega, d, \epsilon)$ to be the maximum number of points in an $\epsilon$-separated subset of $\Omega$ with respect to $d$.

Szarek uses two essential quantities to obtain the metric entropy estimates in [7]. The first is $\kappa(M)$, the operator norm of the orthogonal projection onto $\mathcal{X}$ where the domain and range of the projection are equipped with the operator norm. The second quantity that Szarek employs are the weaving numbers of $X$. We will use a slightly modified version of this. The change is based on Szarek's preference to use the geodesic metric on $X$ and my inclination to use the extrinsic norm metric. They are the same for our purposes. Given $\theta>0, H$ is $\left(\theta,|\cdot|_{\infty}\right)$-woven if for $u \in H,|u-I|_{\infty}<\theta \Rightarrow \exists h \in \mathcal{H}$ such that $|h|_{\infty}<\frac{\pi}{16}$ and $e^{h}=u$. We define $\theta(X)$ to be the supremum over all $\theta$ satisfying the preceding condition.

We now state the main result of Szarek's ([7]), slightly altered in our new notation.

Theorem 8.1. Suppose $\beta \in(0,1 / 2]$ and $\min \left\{\theta(X), \kappa(X)^{-1}\right\} \geq \beta$. Assume that one of the following conditions holds:

- $\operatorname{dim} H \leq(1-\beta) k^{2}$.

- There exists a subspace $E \subset \mathbb{C}^{k}$ invariant under $H$ with $\operatorname{dim} E>\beta k$ satisfying $\beta k \leq \operatorname{dim} E \leq(1-\beta) k$.

- There exists a subspace $E \subset \mathbb{C}^{k}$ invariant under $H$ with $p=\operatorname{dim} E>\beta k$ such that the decomposition $\mathbb{C}^{k}=E \oplus E^{\perp}$ induces an isomorphism $H \rightarrow$ $U(p) \times H_{o}$ for some subgroup $H_{o}$ of $U_{k-p}$.

Then for any $\epsilon \in(0, \beta / 4)$,

$$
\left(\frac{c}{\epsilon}\right)^{\operatorname{dim} X} \leq N\left(X, d_{\infty}, \epsilon\right) \leq\left(\frac{C}{\epsilon}\right)^{\operatorname{dim} X}
$$

where $c, C>0$ are constants depending only on $\beta$.

The utility of Szarek's result lies in the fact that the quantities $c$ and $C$ depend only on $\beta$. We now provide the proof of Lemma 3.5.

Proof of Lemma 3.5. Suppose $H$ is tractable. Consider the conditional expectation $e: M_{k}(\mathbb{C}) \rightarrow H^{\prime \prime} . \quad I-e$ restricted to $\mathcal{G}$ is the orthogonal projection onto $\mathcal{X}$ and since $\|e\| \leq 1$, it follows that $\kappa(X)^{-1} \geq \frac{1}{2}$. The spectral theorem shows that $\theta(X)>\left|e^{i \frac{\pi}{16}}-1\right|$. Hence, $\min \left\{\theta(X), \kappa(X)^{-1}\right\}>\frac{1}{20}$. I claim that $H$ satisfies one of the three conditions as stated in the theorem for $\beta=\frac{1}{20}$. Without loss of generality, assume $H$ takes the form appearing in the definition of a tractable Lie subgroup of $U_{k}$. Suppose there exist some $1 \leq j_{1}, \ldots, j_{q} \leq m$ such that $\frac{k}{20} \leq \sum_{i=1}^{q} k_{j_{i}} l_{j_{i}} \leq \frac{19 k}{20}$. Then $H$ satisfies the second condition of Theorem 8.1. Otherwise there must exist some $1 \leq i \leq m$ for which $k_{i} l_{i}>\frac{19 k}{20}$. If $k_{i}=1$, then $H$ satisfies the third condition of the theorem. Otherwise $k_{i}>1$ and this forces there to be a reducing subspace $E$ for $H$ with $\frac{19 k}{60} \leq \operatorname{dim} E \leq \frac{k}{2}$ whence $H$ fulfills the second condition of the theorem. Theorem 8.1 now yields the desired result.

Having dealt with Lemma 3.5, let us turn to the finite-dimensional situation in Lemma 5.2. More generally first consider the viability of the lower bounds of Theorem 8.1 when $X$ is obtained from tractable $H$ and where instead of using $d_{\infty}$ we 
use $d_{2}$. Some results of [7] work for unitarily invariant norms and metrics but with the $|\cdot|_{2}$-norm problems arise. The quantity $\theta(X)$, properly interpreted, does not stay uniformly away from 0 even when we consider the homogeneous spaces in Section 5 associated to a finite-dimensional $M$. Presumably $X$ would be $\left(\theta,|\cdot|{ }_{2}\right)$-woven if for $u \in H,|u-I|_{2}<\theta \Rightarrow \exists h \in \mathcal{H}$ such that $|h|_{\infty}<\frac{\pi}{16}$ and $e^{h}=u$. Unfortunately, the homogeneous spaces to which we will restrict our attention (which class is much smaller than the class of homogeneous spaces obtained from tractable $H$ ) will fail to have $\theta$ values uniformly bounded away from 0 . Nevertheless, we still have the key result [7], Lemma 10, where the use of $\theta(X)$ was crucial:

Lemma 8.2. There exist $\lambda, r>0$ such that for any $k \in \mathbb{N}$ and tractable $H$ of $U_{k}$, if $x, y \in \mathcal{X}$, and $|x|_{\infty},|y|_{\infty}<r$, then

$$
d_{2}\left(q\left(e^{x}\right), q\left(e^{y}\right)\right) \geq \lambda|x-y|_{2}
$$

where $q: U_{k} \rightarrow X$ is the quotient map.

Proof. For $r$ (as yet to be specified) and any such $x$ and $y$ as above, there exists by definition an $h \in \mathcal{H}$ with $|h|_{\infty} \leq \pi$ satisfying

$$
d_{2}\left(q\left(e^{x}\right), q\left(e^{y}\right)\right)=\inf _{v \in H}\left|e^{-y} e^{x}-v\right|_{2}=\left|e^{-y} e^{x}-e^{h}\right|_{2} .
$$

Set $u=e^{-y} e^{x}$. By the spectral theorem write $h=i \sum_{j=1}^{d} \beta_{j} f_{j}$ where the $f_{j}$ are mutually orthogonal projections and the $\beta_{j}$ are real numbers. We can arrange it so that for each $j, i f_{j} \in \mathcal{H}$, i.e., $h$ takes the block form of $\mathcal{H}$. Define $\gamma_{j}$ to be $4 r$ if $\beta_{j}>4 r,-4 r$ if $\beta_{j}<-4 r$, and $\beta_{j}$ if $\left|\beta_{j}\right| \leq 4 r$. Set $z=i \sum_{j=1}^{d} \gamma_{j} f_{j} \in \mathcal{H} .|z|_{\infty} \leq 4 r$. Define $\Lambda_{1}=\left\{j \in \mathbb{N}: 1 \leq j \leq d,\left|\beta_{j}\right| \leq 4 r\right\}$ and $\Lambda_{2}=\{1, \ldots, d\}-\Lambda_{1}$. Observe that $|x|_{\infty},|y|_{\infty}<r \Rightarrow|u-I|_{\infty}<2 r$. Then

$$
\begin{aligned}
\left|u-e^{z}\right|_{2}^{2} \leq \sum_{j=1}^{d}\left|u f_{j}-e^{i \gamma_{j}} f_{j}\right|_{2}^{2} & =\sum_{j \in \Lambda_{1}}\left|u f_{j}-e^{i \beta_{j}} f_{j}\right|_{2}^{2}+\sum_{j \in \Lambda_{2}}\left|u f_{j}-e^{i \gamma_{j}} f_{j}\right|_{2}^{2} \\
& \leq\left|u-e^{h}\right|_{2}^{2}+\sum_{j \in \Lambda_{2}}(6 r)^{2}\left|f_{j}\right|_{2}^{2} .
\end{aligned}
$$

Now for $r$ sufficiently small, $3 r \leq\left|1-e^{i 4 r}\right|$ ( $r$ dependent only upon the exponential map),

$$
\begin{aligned}
\sum_{j \in \Lambda_{2}}(6 r)^{2}\left|f_{j}\right|_{2}^{2} & \leq \sum_{j \in \Lambda_{2}} 36\left(\left|1-e^{i 4 r}\right|-2 r\right)^{2}\left|f_{j}\right|_{2}^{2} \\
& \leq \sum_{j \in \Lambda_{2}} 36\left(\left|1-e^{i \beta_{j}}\right|-2 r\right)^{2}\left|f_{j}\right|_{2}^{2} \\
& \leq \sum_{j \in \Lambda_{2}} 36\left(\left|f_{j}-e^{i \beta_{j}} f_{j}\right|_{2}-\left|f_{j}-u f_{j}\right|_{2}\right)^{2} \\
& \leq \sum_{j \in \Lambda_{2}} 36\left|u f_{j}-e^{i \beta_{j}} f_{j}\right|_{2}^{2} \\
& \leq 36\left|u-e^{h}\right|_{2}^{2} .
\end{aligned}
$$

It follows that $\left|u-e^{z}\right|_{2} \leq 7\left|u-e^{h}\right|_{2}$.

A repetition of the proof of Lemma 10 in [7] minus the parts referring to $\theta(X)$ shows that there exist $\lambda, r>0$ (which we can make as small as we want and in particular have $r$ satisfy $\left.3 r \leq\left|1-e^{i 4 r}\right|\right)$ independent of the tractable $H$ such that 
for any $x, y \in \mathcal{X}$ with $|x|_{\infty},|y|_{\infty}<r,\left|e^{-y} e^{x}-e^{z}\right|_{2} \geq \lambda|x-y|_{2}$. By what preceded for any $x, y \in \mathcal{X}$ with $|x|_{\infty},|y|_{\infty}<r$,

$$
d_{2}\left(q\left(e^{x}\right), q\left(e^{y}\right)\right)=\left|e^{-y} e^{x}-e^{h}\right|_{2} \geq \frac{\left|e^{-y} e^{x}-e^{z}\right|_{2}}{7} \geq \frac{\lambda|x-y|_{2}}{7} .
$$

The result above does not quite provide the desired lower bounds for the homogeneous space associated to $H$. Observe that if all the normalized Hilbert-Schmidt quantities are replaced with operator norm quantities, then $P\left(X, d_{\infty}, \epsilon\right)$ is bounded below by the $\frac{\epsilon}{\lambda}$ packing number of the $r$-ball of the space $\mathcal{X}$ (endowed with $|\cdot|_{\infty}$ ). The appropriate lower bounds for packing numbers of balls in finite-dimensional spaces can be obtained through a standard volume comparison argument (see [1] for an example of how this technique yields the packing number bounds). Indeed, this is how the lower bound is achieved in Theorem 8.1. The result above is not quite the same. It shows that $P\left(X, d_{2}, \epsilon\right)$ dominates the $\frac{\epsilon}{\lambda}$ packing number with respect to the $|\cdot|_{2}$-metric of the ball of $|\cdot|_{\infty}$-radius $r$ in $\mathcal{X}$. The issue is that we have a lower bound involving two different metrics. We want to obtain the appropriate lower bounds by using the volume comparison argument, but our task is slightly complicated by this. We must now examine the ratio of the volumes of balls of radius 1 with respect to $|\cdot|_{\infty}$ and $|\cdot|_{2}$ in the space $\mathcal{X}$ associated to $H$.

Despite the difficulties mentioned, Lemma 5.2 demands lower bounds on the packing numbers of a specific class of homogeneous spaces, in fact, much smaller than the class of all homogeneous spaces obtained from tractable subgroups. Hence, the task at hand is not so daunting. With Lemma 8.2 in hand we now begin the main part of the proof of Lemma 5.2. As discussed at the end of the preceding paragraph, our main objective is to examine the ratio of the volumes of balls in the orthogonal complements of certain Lie subalgebras.

Proof of Lemma 5.2. Maintain all the assumptions made on $M$ and $\varphi$ in Section 5. We assume that $M \neq \mathbb{C} I$ since Lemma 8.2 clearly holds in this situation. There exist constants $1>\delta, c_{1}, c_{2}>0$ such that if $\delta>\varepsilon>0$ and $r_{1}, \ldots, r_{p} \in \mathbb{R}$ satisfy $\left|r_{j}-\frac{\alpha_{j}}{n_{j}}\right|<\varepsilon$ for all $j$, then $c_{1}<\sum_{j=1}^{p} r_{j}^{2}<c_{2}\left(M \neq \mathbb{C} I \Rightarrow \sum_{j=1}^{p}\left(\frac{\alpha_{j}}{n_{j}}\right)^{2}<1\right)$. Now suppose $\frac{1}{2} \cdot \min \left\{\delta, 1-c_{1}, \alpha_{1}, \ldots, \alpha_{p}\right\}>\varepsilon>0$. It is a trivial consequence of Lemma 3.6 that there exists a sequence $\left\langle\sigma_{k}\right\rangle_{k=1}^{\infty}$ such that for each $k, \sigma_{k}: M \rightarrow M_{k}(\mathbb{C})$ is a $*$-homomorphism and for $k$ sufficiently large:

- $\left\|t r_{k} \circ \sigma_{k}-\varphi\right\|<\varepsilon$.

- The set of unitaries $H_{k}$ of $\sigma_{k}(M)^{\prime}$ is a tractable Lie subgroup of $U_{k}$ and setting $X_{k}=U_{k} / H_{k}$ we have that $k^{2}\left(\triangle_{\varphi}(M)-\varepsilon\right) \leq \operatorname{dim}\left(X_{k}\right)$.

We must demonstrate the third item in Lemma 5.2 (the lower bound packing estimate) and make sure that the constant $\kappa$ obtained is independent of $\varepsilon$ and $\epsilon$. It can be arranged so that there exists a $k_{0} \in \mathbb{N}$ such that for all $k \geq k_{0}$ the representation $\sigma_{k}$ takes the simple form described in the proof of Lemma 3.6. Recall from the proof of Lemma 3.6 that for each $k \geq k_{0}$ we have the $l_{1}(k), \ldots, l_{p+1}(k)$, associated to $\sigma_{k}$. Moreover, for such $k$, the construction of the $\sigma_{k}$ in Lemma 3.6 and the bound placed on $\varepsilon$ shows that $\frac{3}{2} \alpha_{j}>\frac{l_{j}(k) n_{j}}{k}>\frac{\alpha_{j}}{2}$ for each $j, c_{1} k^{2}<\sum_{j=1}^{p+1} l_{j}(k)^{2}<c_{2} k^{2}$, and $l_{p+1}(k)<n_{1} \cdots n_{p}$ for $k>k_{0}$.

For each $k$ denote $\mathcal{H}_{k}$ and $\mathcal{X}_{k}$ to be the spaces $\mathcal{H}$ and $\mathcal{X}$ associated to $H=H_{k}$. Again, we have translated the packing number problem (the third condition of 
Lemma 5.2) into the problem of comparing the volumes of the balls of $\mathcal{X}_{k}$ with respect to the norms $|\cdot|_{\infty}$ and $|\cdot|_{2}$ and finding an appropriate relationship between the two values for sufficiently large $k$.

For any $r>0$ denote by $\mathcal{G}_{k}^{r}, \mathcal{H}_{k}^{r}$, and $\mathcal{X}_{k}^{r}$ the balls centered at the origin of operator norm less than or equal to $r$ in $i M_{k}^{s a}(\mathbb{C}), \mathcal{H}_{k}$, and $\mathcal{X}_{k}$, respectively. Consider the conditional expectation $e$ for $H_{k}^{\prime \prime}$. Define $\Phi: i M_{k}^{s a}(\mathbb{C}) \rightarrow \mathcal{H}_{k} \bigoplus \mathcal{X}_{k}$ by $\Phi(x)=(e(x),(I-e) x)$. Since $e$ is a contraction when its domain and range are endowed with the operator norm, it follows that $\Phi\left(\mathcal{G}_{k}^{1}\right) \subset \mathcal{H}_{k}^{1} \times \mathcal{X}_{k}^{2} . \Phi$ is an isometry when its domain and range are endowed with the Hilbert-Schmidt norm (normalized or not). Thus, $\operatorname{vol}\left(\mathcal{G}_{k}^{1}\right)=\operatorname{vol}\left(\Phi\left(\mathcal{G}_{k}^{1}\right)\right) \leq \operatorname{vol}\left(\mathcal{H}_{k}^{1}\right) \cdot \operatorname{vol}\left(\mathcal{X}_{k}^{2}\right)$. Notice that here we calculate the volumes of $\mathcal{H}_{k}^{1}$ and $\mathcal{X}_{k}^{2}$ in their ambient Hilbert spaces $\mathcal{H}_{k}$ and $\mathcal{X}_{k}$ endowed with the real inner product $R e T r$.

We claim that if for each $d, \Lambda_{d}=\operatorname{vol}\left(\mathcal{G}_{d}^{1}\right)$ and $\Theta_{d}$ denotes the volume of the ball of radius $\sqrt{d}$ in $\mathbb{R}^{d^{2}}$, then there exists some constant $1>\zeta_{1}>0$ such that $\left(\zeta_{1}\right)^{d^{2}} \leq \frac{\Lambda_{d}}{\Theta_{d}}$ for all $d$. By [5] there exists a constant $c>0$ such that $\frac{a_{d}}{b_{d}} \sim(c)^{2 d^{2}}$ as $d \rightarrow \infty$ where $a_{d}$ is the volume (with respect to the real inner product $\operatorname{Re} T r$ ) of the operator norm unit ball of $M_{k}(\mathbb{C})$ and $b_{d}$ is the volume of the ball of radius $\sqrt{2 d}$ in $2 d^{2}$-dimensional real Euclidean space. We now use the same trick in the preceding paragraph. Decompose $M_{k}(\mathbb{C})$ as the orthogonal direct sum $M_{k}^{s a}(\mathbb{C}) \oplus i M_{k}^{s a}(\mathbb{C})$. It follows that the operator norm unit ball of $M_{k}(\mathbb{C})$ is contained in the direct sum of the operator norm unit ball of $M_{k}^{s a}(\mathbb{C})$ and the operator norm unit ball of $\mathcal{G}_{d}^{1}$. The volume of this latter set is $\left(\Lambda_{d}\right)^{2}$. So $\frac{a_{d}}{b_{d}}<\frac{\left(\Lambda_{d}\right)^{2}}{b_{d}} \sim \frac{\left(\Lambda_{d}\right)^{2}}{\left(\Theta_{d}\right)^{2}}$ and this yields the desired result.

Now $\operatorname{vol}\left(\mathcal{H}_{k}^{1}\right)<\Theta_{l_{1}(k)} \cdots \Theta_{l_{p+1}(k)} \cdot \sqrt{n_{1} \cdots n_{p}}$ and because $\operatorname{dim} \mathcal{X}_{k}>\left(1-c_{2}\right) \cdot k^{2}$ for sufficiently large $k$, it follows that there exists a $\zeta_{2}>0$ (dependent only on $n_{1}, \ldots, n_{p}, \zeta_{1}$ and $c_{1}$ ) such that for sufficiently large $k$,

$$
\operatorname{vol}\left(\mathcal{X}_{k}^{2}\right) \geq \frac{\operatorname{vol}\left(\mathcal{G}_{k}^{1}\right)}{\operatorname{vol}\left(\mathcal{H}_{k}^{1}\right)} \geq \frac{\Theta_{k}}{\Theta_{l_{1}(k)} \cdots \Theta_{l_{p+1}(k)}} \cdot \zeta_{2}^{\operatorname{dim} \mathcal{X}_{k}}
$$

$\frac{l_{j}(k)}{k}>\frac{\alpha_{j}}{2 n_{j}}, l_{p+1}(k)<n_{1} \cdots n_{p}$ for $k>k_{0}$, and $\left(1-c_{2}\right) \cdot k^{2}<\operatorname{dim} \mathcal{X}_{k}<\left(1-c_{1}\right) \cdot k^{2}$ for $k>k_{0}$. These three facts and Stirling's formula show that there exists a constant $\zeta_{3}>0$ (dependent only on the $\alpha_{i}, n_{i}, c_{1}$, and $c_{2}$ ) such that for sufficiently large $k$ the dominated term above is greater than or equal to $\left(\zeta_{3}\right)^{\operatorname{dim} \mathcal{X}_{k}} \cdot C_{\operatorname{dim} \mathcal{X}_{k}}$ where $C_{\operatorname{dim} \mathcal{X}_{k}}$ is the volume of the ball of radius $\sqrt{k}$ in $\mathbb{R}^{\operatorname{dim} \mathcal{X}_{k}}$. Notice that this quantity is the volume of the ball of $\mathcal{X}_{k}$ of $|\cdot|_{2}$ radius 1 . In other words there exists a $\zeta>0$ (again dependent only on the $\alpha_{i}, n_{i}, c_{1}$, and $c_{2}$ ) such that for sufficiently large $k$,

$$
\frac{\operatorname{vol}\left(\mathcal{X}_{k}^{1}\right)}{C_{\operatorname{dim} \mathcal{X}_{k}}}>(\zeta)^{\operatorname{dim} \mathcal{X}_{k}}
$$

The standard volume comparison method (for an example of how this method is used see [1]) shows that for such $k$ and any $\epsilon>0, P\left(\mathcal{X}_{k}^{r},\left.|\cdot|\right|_{2}, \epsilon\right)>\left(\frac{r \zeta}{\epsilon}\right)^{\operatorname{dim} \mathcal{X}_{k}}$. Using Lemma 8.2 it follows that for $k$ sufficently large, if $\epsilon>0$, then

$$
P\left(X_{k}, d_{2}, \epsilon\right)>\left(\frac{\lambda r \zeta}{\epsilon}\right)^{\operatorname{dim} X_{k}}
$$


Set $\kappa=\lambda r \zeta$. Then $\kappa$ depends only on $\alpha_{i}, n_{i}, c_{1}, c_{2}$, and the upper bound placed on $\varepsilon$. The upper bound can be relaxed for the purposes of Lemma 5.2. We have the third and final condition of Lemma 5.2.

\section{ACKNOWLEDGEMENTS}

I thank Dan Voiculescu, my advisor, both for suggesting that I work on computing the free entropy dimension of $M_{2}(\mathbb{C})$ and for providing helpful comments throughout. Also I thank Stanislaw Szarek for the exchanges and suggestions which formed the core of the proof of Lemma 5.2 in the Addendum. I am grateful to Charles Holton for the simplification of the proof of Corollary 7.1 and the useful conversations on Minkowski dimension. Finally, this work would not have been possible without the generous contributions of the NSF Graduate Fellowship program.

\section{REFERENCES}

[1] Carl, B. and Stephani, I. Entropy, Compactness, and the Approximation of Operators, Cambridge Tracts in Mathematics, No. 98, Cambridge University Press, Cambridge, 1990. MR 92e: 47002

[2] Dykema, K. J., Free products of hyperfinite von Neumann algebras and free dimension, Duke Math. J. 69 (1993), 97-119. MR 93m:46071

[3] Ge, Liming, Applications of free entropy to finite von Neumann algebras, II, Annals of Mathematics, 147 (1998), 143-157. MR 99c:46068

[4] Ge, Liming and Shen, Junhao, On free entropy dimension of finite von Neumann algebras, Geometric and Functional Analysis, Vol. 12, (2002), 546-566.

[5] Raymond, Jean Saint, Le volume des idéaux d'opérateurs classiques, Studia Mathematica, vol. LXXX (1984), 63-75.

[6] Stefan, M., The indecomposability of free group factors over nonprime subfactors and abelian subalgebras, preprint.

[7] Szarek, S. Metric entropy of homogeneous spaces, Quantum Probability (Gdansk, 1997), Banach Center Publications vol. 43, Polish Academy of Science, Warsaw, 1998, 395-410. MR 2000c:53097

[8] Voiculescu, D., Dykema, K.J., and Nica, A. Free Random Variables, CRM Monograph Series, v. 1, American Mathematical Society, Providence, RI, 1992. MR 94c:46133

[9] Voiculescu, D., The analogues of entropy and of Fisher's information measure in free probability theory, II, Inventiones Mathematicae 118, (1994), 411-440. MR 96a:46117

[10] Voiculescu, D., The analogues of entropy and of Fisher's information measure in free probability theory III: The Absence of Cartan Subalgebras, Geometric and Functional Analysis, Vol. 6, No.1 (1996), 172-199. MR 96m:46119

[11] Voiculescu, D., A strengthened asymptotic freeness result for random matrices with applications to free entropy, Internat. Math. Res. Notices 1 (1998), 41-63. MR 2000d:46080

Department of Mathematics, University of California, Berkeley, California 947203840

E-mail address: factor@math.berkeley.edu 